Article

\title{
Cosmic-Ray Database Update: Ultra-High Energy, Ultra-Heavy, and Antinuclei Cosmic-Ray Data (CRDB V4.0)
}

\author{
David Maurin ${ }^{1, *}$ (1), Hans Peter Dembinski ${ }^{2, *}$ (]) Javier Gonzalez ${ }^{3}$, Ioana Codrina Mariş 4 (]) \\ and Frédéric Melot ${ }^{1}$ \\ 1 LPSC, Université Grenoble Alpes, CNRS/IN2P3, 53 avenue des Martyrs, 38026 Grenoble, France; \\ frederic.melot@lpsc.in2p3.fr \\ 2 Dortmund Technical University, Experimental Physics 5, Otto-Hahn-Strasse 4a, 44227 Dortmund, Germany \\ 3 Harvard-Smithsonian Center for Astrophysics, 60 Garden Street, Cambridge, MA 02138, USA; \\ javierggt@yahoo.com \\ 4 Université Libre de Bruxelles, Faculté des Sciences, Département de Physique, Campus La Plaine, \\ Boulevard du Triomphe, 2 CP 230, 1050 Bruxelles, Belgium; Ioana.Maris@ulb.ac.be \\ * Correspondence: david.maurin@lpsc.in2p3.fr (D.M.); hans.dembinski@tu-dortmund.de (H.P.D.)
}

Received: 2 June 2020; Accepted: 15 July 2020; Published: 24 July 2020

\begin{abstract}
We present an update on CRDB, the cosmic-ray database for charged species. CRDB is based on MYSQL, queried and sorted by jquery and table-sorter libraries, and displayed via PHP web pages through the AJAX protocol. We review the modifications made on the structure and outputs of the database since the first release (Maurin et al., 2014). For this update, the most important feature is the inclusion of ultra-heavy nuclei $\left(\mathrm{Z}>30\right.$ ), ultra-high energy nuclei (from $10^{15}$ to $10^{20} \mathrm{eV}$ ), and limits on antinuclei fluxes ( $Z \leq-1$ for $A>1$ ); more than 100 experiments, 350 publications, and 40,000 data points are now available in CRDB. We also revisited and simplified how users can retrieve data and submit new ones. For questions and requests, please contact crdb@lpsc.in2p3.fr.
\end{abstract}

Keywords: astroparticle; cosmic rays; databases

\section{Introduction}

Cosmic-ray (CR) physics has been established more than a century ago and progress in the measurements has been performed through this entire period. Besides preserving the measurements for their historical value, the motivations for a CR database are numerous.

Firstly, GeV CRs vary with the Solar cycle, and past data are still of interest-for an illustration of the usage of CR data going back to the 1950s, see Ghelfi et al. [1]. Secondly, it is infeasible to design and build CR experiments that measure all species at all energies, so that multi-species studies over different energy ranges have to rely on many datasets from as many experiments-for an illustration of the usage of data samples of $\mathrm{H}$ to Ni elements over 45 years, see Shen et al. [2]. Thirdly, new synergies with other fields of astrophysics make the collection of even the rarest CR data desired. For instance, ultra-heavy elements (UHCRs, $Z>30$ ) have very small fluxes and are difficult to measure. They require dedicated experiments and so far the data are very sparse (e.g., Donnelly et al. [3], Binns et al. [4]). UHCRs have not been a very active topic in the last decade, but the situation is likely to change: the first detection of gravitational waves from a binary neutron star inspiral [5] —and in particular optical follow-ups-indicated that neutron star mergers could be a major contributor to r-process nucleosynthesis for UHCRs [6,7]. Fourthly, in the last two decades, a growing number of high-precision experiments were developed to measure leptons, antimatter, and $Z<30$ nuclei in tens of $\mathrm{MeV}$ to hundreds of TeV range (AMS-02, PAMELA, TRACER, etc.). These balloon-borne or space-based 
experiments allow us to address many astrophysics questions (e.g., [8]) including searches for dark matter (e.g., [9]). In the context of these searches, upper limits on antinuclei were improved [10] and efforts are growing towards the first detection of CR antideuterons [11]. A few puzzling $40 \mathrm{GeV}$ events compatible with ${ }^{3} \overline{\mathrm{He}}$ were reported by the AMS-02 collaboration [12]. Adding previously reported upper limits on antinuclei (on various energy ranges) in a database is thus also timely.

A central motivation for a comprehensive CR database is the energy coverage. CR experiments based on direct detection have recently shown a spectral feature in several species at a few hundreds of GV [13-15]. Other spectral features have been observed at higher energies: the knee (at a few PeV), the ankle (around $5 \mathrm{EeV}$ ), and the toe (flux suppression above $100 \mathrm{EeV}$ ); a fainter second knee around $200 \mathrm{PeV}$ was also observed more recently [16]. These features were observed with ground-based indirect CR experiments (KASCADE-Grande, Pierre Auger Observatory, Telescope Array, IceCube, TUNKA, and many more), which do not resolve CR species individually. Weak spectral features can be confirmed and enhanced by combining data from multiple experiments. Gathering data that cover the full CR spectrum can help to shed light on the Galactic to extragalactic transition (e.g., Globus et al. [17], Thoudam et al. [18]) and on the possible sources for the most energetic CR events ever observed (e.g., [19]).

A central shared database offers multiple advantages for research. It enables studies, which would otherwise be too time-consuming or error-prone, by assuring data quality, completeness, and traceability. In principle, it can also allow for a pooling of human resources (from the CR community) to fill and maintain it. Electronic resources to gather and disseminate data became available in the 1990s, but CR databases appeared much later. For galactic CR (GCR) data, the momentum was provided by teams involved in the development of GCR propagation codes. The GALPROP ${ }^{1}$ team started to distribute CR data as a simple ASCII file [20]. As part of the development of the USINE ${ }^{2}$ propagation code [21], our team independently gathered these data and set up the first CR database, called CRDB (Maurin et al. [22]); our database comes along with a contextualised presentation of the data and experiments, ASCI I file exports (compliant to GALPROP and USINE formats), and online plot generation. Shortly after, the Italian Space Agency (ASI) developed a CR database to host originally ASI-supported experimental data [23]. The design and functionality of the CRDB-ASI ${ }^{3}$ was strongly influenced by the $\mathrm{CRDB}$, following discussions between our teams. Since then, the CRDB and CRDB-ASI have been independently developed further. The CRDB-ASI is not as comprehensive as the current version of the $\mathrm{CRDB}$, but it offers specialised datasets (trapped events and Solar Energetic Events) absent in our database. For ultra high-energy CRs (UHECRs, above a PeV), the first initiative to build a common database came from the KCDC project ${ }^{4}$ [24]. The primary goal of KCDC is to offer researchers open access to raw data of the KASCADE and KASCADE-Grande experiments. It also contains a collection of high-energy CR flux measurements from more than 20 experiments published between 1984 and 2020 (contrarily to CRDB, an account is required to access KCDC data).

This paper discusses CRDB $v 4.0$, a major upgrade of the database in terms of its data content, user interfaces, and data submission forms. The paper is organised as follows: Section 2 recalls important definitions and the database structure; Section 3 highlights the specifics of the data added in this version; Section 4 briefly goes over the web interfaces and outputs, focusing on the new and simpler data submission form and the updated REST and Solar modulation time series interfaces. We conclude and discuss possible CRDB extensions in Section 5. We gather in Appendix A useful tips and caveats (on extracted data) for $\mathrm{CRDB}$ users, and we provide in Appendix B sorted lists of all experiments and publications in CRDB $\mathrm{V} 4.0$.

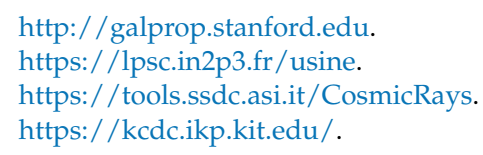




\section{Database Structure and Updates}

Throughout the paper, we separate the data in two broad categories, data and meta-data (data about data). Clearly separating these two categories is important to define the format to submit new data (see Section 3). The categories comprise the following items:

- Data: CR data points, including energy range and data uncertainties;

- Meta-data: CR data-related informations that include data taking periods, experiments that provide the data, publication from which the data were taken, etc.

The first CRDB publication [22] focused on the rationale behind the design choices made for the database structure and organisation (in MYSQL). In this update, we follow a complementary approach. We start at the data level, moving up to the experiments and then publications, underlining some salient aspects of the data themselves and how they are tied to the other meta-data tables. Along the way, we highlight the changes made between CRDB V2.1 [22] and CRDB V4.0.

The database structure is shown in Figure 1. By design, each entry has a unique ID in all tables, so that all tables can be accessed by other tables; this is particularly useful to build bridge tables (i.e., building associations between table entries). The different tables, their contents, and the associations between data and meta-data tables are further detailed in the following subsections.

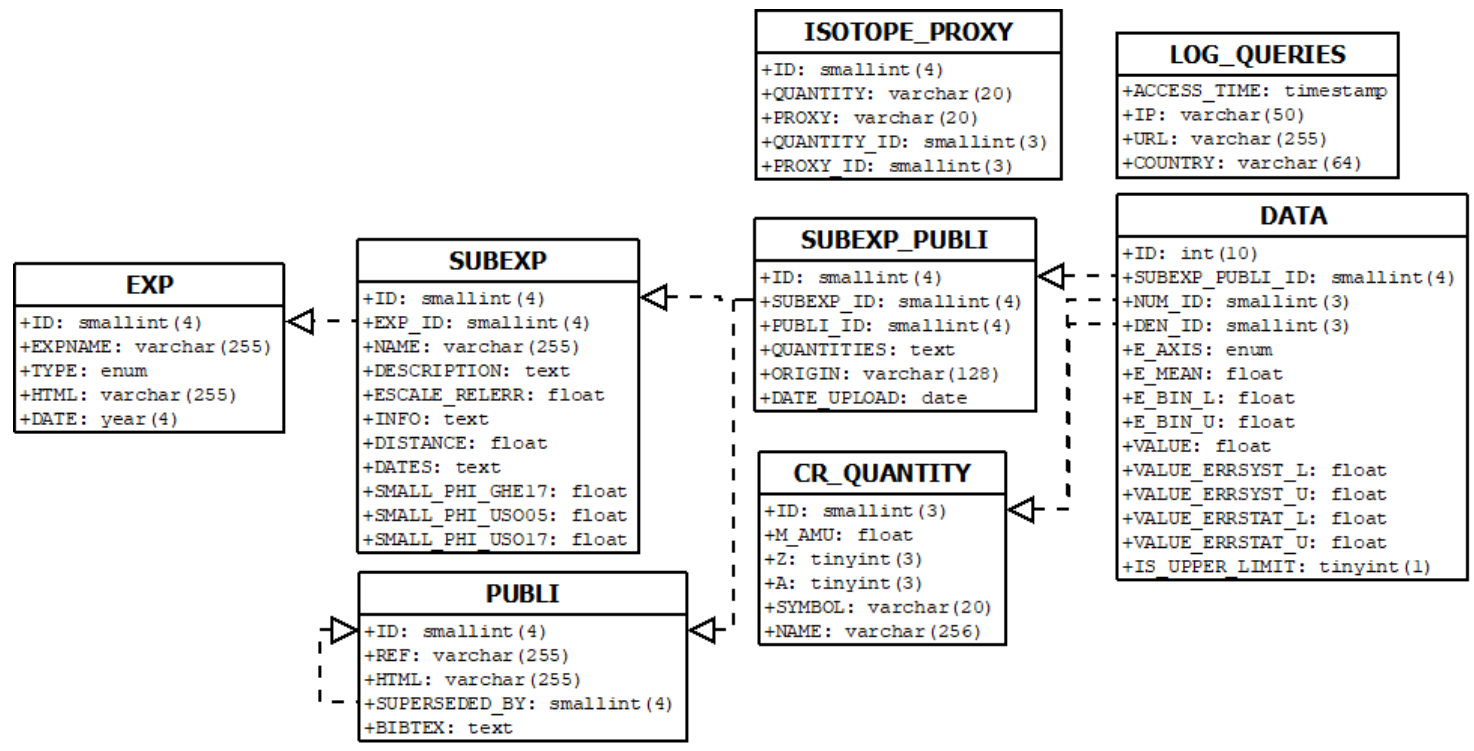

Figure 1. Tables and keys of the database MYSQL structure. The data are stored in the DATA table (Section 2.1) with CR names defined in the CR_QUANTITY table. The meta-data related to experiments are stored in EXP (Section 2.2.1) and SUBEXP tables (Section 2.2.2), while those related to publication are stored in the PUBLI table (Section 2.3.1); a bridge table, SUBEXP_PUBLI (Section 2.3.2), links all meta-data. The LOG_QUERIES table is used to track the number of visits in CRDB (Section 4.1.4). The ISOTOPE_PROXY table is required for energy-axis conversions of CR fluxes (Appendix A.4).

\subsection{DA TA Table}

A data point corresponds to a quantity measurement (with uncertainties) at a given energy. We list below the keys required for a full data entry description in the DATA table (see Figure 1). The only change made since CRDB V2. 1 is enabling upper limits data (new key IS_UPPER_LIMIT).

- ID: unique identifier for a data.

- NUM and DEN: a data quantity is a single item (NUM) or a ratio of two items (NUM and DEN). The former usually corresponds to a measured flux (in unit of $E_{\text {unit }}^{-1} \mathrm{~m}^{-2} \mathrm{~s}^{-1} \mathrm{sr}^{-1}$, with $E_{\text {unit }}$ in $\mathrm{GeV}, \mathrm{GeV} / \mathrm{n}$, or $\mathrm{GV}$ in $\mathrm{CRDB}$ ) and the latter to a ratio of fluxes (no unit). Depending on the 
detector charge and isotopic resolution, the measured quantities are isotopes (e.g., ${ }^{12} \mathrm{C}$ ), elements (e.g., C), or even groups of elements; the latter case mostly applies for UHCR and UHECR data (see next sections). Indirect detection CR experiments also provide shower-related quantities (with their specific units) that we plan to add in the future. To allow for these various possibilities, we define a CR_QUANTITY table which contains a list of names including CR isotope names, element names, and names for relevant groups of elements or other significant measurable quantities (e.g., Allparticles, see Table A2 for new names in CRDB v4.0).

- E-AXIS: the database energy types are ETOT, EK, R, EKN, and ETOTN. In CRDB, they correspond to the total $\left(E_{\text {tot }}\right)$ and kinetic energy $\left(E_{\mathrm{k}}=E_{\text {tot }}-m\right)$ set in $\mathrm{GeV}$, rigidity $(\mathcal{R}=p /(Z e))$ in $\mathrm{GV}$, kinetic energy per nucleon $\left(E_{\mathrm{k} / \mathrm{n}}=E_{k} / A\right)$ in $\mathrm{GeV} / \mathrm{n}$, and total energy per nucleon $\left(E_{\mathrm{tot} / \mathrm{n}}=E_{\mathrm{tot}} / A\right)$ in $\mathrm{GeV} / \mathrm{A}$. Depending on the detector principle (calorimeter, spectrometer, etc.), different energy types are measured in experiments and provided in publications, and in $\mathrm{CRDB}$, the data are always stored as published (see Appendix A.4 for conversion).

- E_MEAN, E_BIN_L, and E_BIN_U: fluxes are measured by counting events in energy bins. In $\mathrm{CRDB}$, we include the energy interval range $\left[E_{\mathrm{lo}}, E_{\mathrm{up}}\right]$ when it is published. The measured flux obtained from a finite bin size neither exactly corresponds to the differential flux at the bin centre or at the mean energy $\langle E\rangle$, unless a correction is applied [25]; when comparing a model to the data, it is thus recommended to integrate the flux model over the bin width instead of using the value at the mean energy [26]. If only a single value $\langle E\rangle$ is provided in the publication, we set $E_{\mathrm{lo}}=E_{\mathrm{hi}}=\langle E\rangle$ in CRDB; conversely, if only the bin range is provided, we set $\langle E\rangle=\sqrt{E_{\mathrm{lo}} E_{\mathrm{hi}}}$.

- VALUE, VALUE_ERRSYST_L, ..., IS_UPPER_LIMIT: a measurement has a value and statistical and systematic uncertainties. In $\mathrm{CRDB}$, we store the value and the possibly asymmetric statistical and systematic uncertainties-see Appendix A.2 for subtleties and caveats concerning the gathering of uncertainties from publications. In $C R D B \vee 4.0$, upper limits with the flag IS_UPPER_LIMIT set to 1 .

- SUBEXP_PUBLI_ID: a datum is always attached to a unique publication and a sub-experiment (see next sections). This key enables to bridge the data ID (this table) to the subexp-publi ID (SUBEXP_PUBLI table, see Section 2.2.2).

\subsection{EXP and SUBEXP Tables and Meta-Data}

$\mathrm{CR}$ data are collections of data points at different energies, from an experiment measuring one or several quantities. In $\mathrm{CRDB}$, for flexibility, we make the distinction between an experiment and sub-experiment (hereafter sub-exp for short). The experiment is defined by its name and starting date. The sub-exp is linked to the experiment, but is more closely related to the data in order to uniquely tag the dataset released by this experiment. The tag is here to inform us on (i) analyses from different data taking periods, (ii) data from the same period but from different analysis techniques or different sub-detectors, (iii) data relying on third-party model assumptions (e.g., using different Monte Carlo generators for air showers in UHECRs).

Experiment and sub-experiment data are meta-data (attached to CR data) stored in the EXP and SUBEXP tables respectively (see Figure 1); they are briefly described below.

\subsubsection{EXP Table}

A single change has been made for $\mathrm{CRDB} \vee 4.0$, related to the experience type (key TYPE, see below).

- ID: unique identifier for an experiment.

- EXPNAME: unique name associated with the main instrument (AMS-01, KASCADE, etc.). Most of the data before the 1990s come from balloons, and to ensure a unique identifier CRDB uses the syntax: Balloon (YYYY) for a single flight, Balloon $(1966,1967, \ldots)$ for balloons flown several 
times, and Balloon $(1967+1968+\ldots)$ for experiments that report only the combined analysis of several flights.

- TYPE: in CRDB V4.0, we allow for three experiment types (balloon, ground, or space) that represent three broad categories of measurement conditions with different caveats and systematic uncertainties. The experiments in the 'Experiments/Data' tab (see Section 4.1.2) on the CRDB website can be sorted according to date, name, and this type.

- DATE and HTML: we also keep track of the starting year of the experiment and a link to the official experiment website is provided (if it exists).

\subsubsection{SUBEXP Table}

The SUBEXP table contains detailed information on possible sub-detector(s) from which the data were obtained; this includes the data taking conditions and, in some cases, the analysis technique. In CRDB v4 . 0, a new key was added to track the energy-scale uncertainty (i.e., a calibration uncertainty on the energy measurement), which is especially important for ground-based experiments $(\gtrsim 10 \%)$. We list all keys in the SUBEXP table (see Figure 1) and then comment on the effect of Solar modulation on multi-GeV GCR data [27]. In particular, we stress how the last three keys listed below are necessary inputs for Solar modulation calculations.

- ID: unique identifier for a sub-experiment.

- EXP_ID: ID of the experiment in the EXP table to which the sub-exp is attached.

- NAME: concatenation of the experiment (or sub-experiment) name with the sub-detector/specific detection technique used (PAMELA-CALO...), or with the air shower MC generator used (IceTop SIBYLL-2.1...), and always with the data taking period (e.g., IMP7 (1973/05-1973/08)).

- DESCRIPTION: rough description of the detection techniques and salient features of the detector (in the sub-experiment). If available from the collaboration, an image of the detector is displayed in the user interface (see Section 4).

- INFO: additional information relevant for the sub-exp. This can be the location of the balloon flight (e.g., one flight McMurdo, Antarctica), some details about the reconstructed data (e.g., Fractions of $\mathrm{H}, \mathrm{He}, \mathrm{O}, \mathrm{Fe}$-like showers in 4-component fit to $\mathrm{X}_{\max }$ data), indication of times series (e.g., Monthly average 2007/03), etc.

- ESCALE_RELERR: if provided in the publication, CRDB V4.0 stores the relative energy-scale uncertainty, $(\Delta E / E)_{\text {scale, }}$ which applies to all energies measured by the sub-experiment ${ }^{5}$. We refer the reader to Appendix A.3 for more details on the origin of this uncertainty.

- DATES: list of data taking periods for the instrument taken from the publication or, if not reported in old balloon flights, retrieved from the STRATOCAT database ${ }^{6}$.

- DISTANCE: most of the experiments are located on Earth, or are orbiting Earth. However, a few satellites (e.g., Ulysses, Voyager, etc.) took data at various locations in the Solar system. We provide in CRDB the average distance to the Sun in a.u. (an astronomical unit is the distance Sun-Earth) during the data taking period, as provided in the publications.

- SMALL_PHI: Accurate Solar modulation models are complex and depend on many parameters. The simplest model, the Force-Field approximation [28-30], depends on a single parameter $\phi(t)$, and remains useful for many applications despite its limitations [31]. Therefore we provide the mean modulation level $\left\langle\phi_{\mathrm{FF}}\right\rangle$ calculated externally from $\phi$ time series (see below) over the sub-experiment data taking periods.

5 In principle, $(\Delta E / E)_{\text {scale }}$ does depend on energy. If future $C R$ publications release energy-dependent $(\Delta E / E)_{\text {scale }}$ their implementation in CRDB will require modifications of the ESCALE_RELERR key.

6 http://stratocat.com.ar/globos/indexe.html. 
From interstellar (IS) to top-of-atmosphere (TOA) fluxes

GCR fluxes are Solar modulated, the modulation level depending on the Solar cycle [32] and the position in the Solar cavity. Interstellar (IS) fluxes refer to fluxes outside of the solar cavity while top-of-atmosphere (TOA) fluxes correspond to modulated fluxes at Earth. The impact of the Solar modulation decreases with energy and can only be disregarded when it becomes much smaller than the data precision ${ }^{7}$. Most balloon and space experiments in CRDB are TOA data from experiments inside the solar cavity. To be compared to them, IS fluxes in GCR propagation models must therefore be Solar modulated. This makes the bookkeeping of the experiment data taking periods and position in the Solar cavity mandatory. These dates and positions can then be fed to dedicated Solar propagation codes-for instance SolarProp ${ }^{8}$ [33] or HelMod ${ }^{9}$ [34]. Alternatively, if CRDB users wish to use the simpler Force-Field approximation [31], they can directly retrieve $\left\langle\phi_{\mathrm{FF}}\right\rangle$ values provided in CRDB.

Average Force-Field levels $\left\langle\phi_{\mathrm{FF}}\right\rangle$ from $\phi(t)$ time series

Modulation time series (in the Force-Field approximation) can be obtained from the analysis $[1,35,36]$ of neutron monitor data ${ }^{10}$. These analyses were pioneered by Usoskin et al. [35] in 2005 and updated in Usoskin et al. [36] in 2017. Following a similar approach, and based on improved propagation of error [37] and interstellar spectra [38], our team independently reconstructed its own $\phi$ time series in Ghelfi et al. [1], roughly finding

$$
\phi_{[\mathrm{Ghe17]}}(t) \approx \phi_{[\mathrm{Uso17}]}(t) \approx \phi_{[\mathrm{Uso} 05]}(t)+100 \mathrm{MV}
$$

In CRDB V4.0, we provide these three values in the SMALL_PHI keys (of the SUBEXP table), tagged as [Uso05], [Uso17], and [Ghe17]. We stress that whereas [Uso05] and [Uso17] are based on monthly-averaged distributed values ${ }^{11}$, [Ghe17] relies on daily averaged values. Independently of the respective merits of these three sets, daily average $\phi$ values are better suited than monthly ones for short duration experiments. Our values [Ghe17] cover all years from 1950 till now and are automatically updated. These values can also be retrieved from the website (see Section 4.2).

\subsection{PUBLI and SUBEXP_PUBLI Tables and Meta-Data}

CR data are published by experimental teams and collaborations in scientific publications. To save time and avoid confusion, we have a strong preference to include only final data published in peer-reviewed journals, and most data in CRDB come from refereed journals. An important exception is the proceedings of the biyearly International Cosmic-Ray Conference (ICRC, started in 1947): until the 1990s, many balloon flight results were published in the ICRC proceedings only, and even nowadays these proceedings still often contain the latest preliminary results and updates of previously published results.

\subsubsection{PUBLI Table}

To store what a publication is, the following keys are used.

- ID: unique identifier for a publication.

- HTML: unique ID for the reference, taken from the Astrophysics Data System $(\text { ADS })^{12}$, e.g., $2014 \mathrm{~A} \& \mathrm{~A}$...569A. .32M.

7 For instance, AMS-02 proton data [13] have a $\lesssim 2 \%$ precision up to a few hundreds of GV, to be compared with a modulation of $\sim 10-20 \%$ at $20 \mathrm{GV}, \sim 2-5$ at $100 \mathrm{GV}$, and at sub-percent above several hundreds of GV.

8 http://www.th.physik.uni-bonn.de/nilles/people/kappl.

9 http://www.helmod.org.

10 http://www01.nmdb.eu.

11 http://cosmicrays.oulu.fi/phi.

12 https://ui.adsabs.harvard.edu/. 
- REF and BIBTEX: these two keys provide a full publication reference and BIBTEX $X^{13}$ entry, automatically retrieved from the ADS ID thanks to the ADS application programming interface (API) ${ }^{14}$.

- SUPERSEDED_BY: in some cases, the same data (same quantity from the same sub-exp) are re-analysed and updated in a subsequent publication. This key enables to store the ID (in the PUBLI table) of the newer publication analysis, keeping track at the same time of deprecated analyses and references. A single level of superseded data can always be seen from the 'Experiments/Data' tab (see Section 4.1).

Deprecated keys in $\mathrm{CRDB} \vee 4.0$

One major modification in the PUBLI table in this release was to abandon book-keeping Solar modulation informations from the publication, i.e., Solar modulation levels calculated by the authors for their data. This feature was introduced in CRDB V2.1-requiring many extra keys in the PUBLI table, see Maurin et al. [22] —in order to highlight the various assumptions behind the modulation levels provided. The motivation was to ease comparisons between modulation levels for data taken at similar periods but provided by different experimental teams. However, picking this information from the publication was time consuming for very little practical use in retrospect ${ }^{15}$. Moreover, as stressed in the previous section, modern CR propagation model studies either make use of $\phi_{\mathrm{FF}}$ or rely on public Solar modulation codes, and the informations stores in the SUBEXP table allow both these practices (see Section 2.2.2). A major benefit of not considering the modulation from the publication is that it simplifies the structure of the database, the extraction of data, and the submission of new data.

\subsubsection{SUBEXP_PUBLI Bridge Table}

To avoid duplicates, for cases where different data refer to the same publication or the same sub-experiment, a bridge table (SUBEXP_PUBLI, see Figure 1) enables to link entries from the SUBEXP and PUBLI tables. Its keys are as follows.

- ID: unique identifier for a subexp-publi association.

- QUAntities: lists all the quantities (fluxes, ratios, etc.) provided by a given sub-exp/publi association.

- DATA_UPLOAD: stores the date at which the corresponding data were uploaded.

- SUBEXP_ID: bridges a sub-experiment/publication result to a 'sub-exp' ID (SUBEXP table).

- $\quad$ PUBLI_ID: bridges a sub-experiment/publication result to a 'publi' ID (PUBLI table).

\section{New Data in CRDB V4.0}

We now turn to the database content. As much as possible, data are added in CRDB on a continuous basis (by one of us); new data can also be submitted (see Section 4.4). Thanks to the feedback of many users, typos and mismatches in the data and meta-data are regularly corrected-the list of corrections and people who helped us (see acknowledgements) are available online (see CRDB log file).

In this section, we highlight the main datasets added since the first release [22], in particular in the two most recent releases CRDB $v 3.1$ (Section 3.1) and $v 4.0$; for the latter, we detail the motivation

13 www.bibtex.org.

14 https://github.com/adsabs/adsabs-dev-api.

15 Indeed, the IS spectra and the Solar modulation models used are too patchy. For IS fluxes, publications used spectra derived from their data, from other authors, of from inputs of GCR propagation models. For Solar modulation, the models found in the publications were (i) for very old data, the outdated diffusion/convection model and its $\eta$ parameter [39-41], (ii) since the 1970s, the still widely used Force-Field approximation and its $\phi_{\text {FF }}$ parameter, or the more evolved spherically symmetric model and its effective $\phi_{1 \mathrm{D}}$ free parameter [42-44], (iii) in more recent studies, the modern sign-charge dependent 3D model with parameters for the current sheet, tilt angle, diffusion tensor, etc. $[27,45,46]$ or, most of the time, no modulation value at all (just the data). 
and specifics of UHCR data (Section 3.2), antinuclei upper limits (Section 3.3), and UHECR data (Section 3.4). The complete list of experiments and publications (presently in CRDB) is gathered in Appendix B.

\subsection{Relevant Data Updates Before CRDB v4.0}

Since the first release of $C R D B$ seven years ago, two major data updates are worth highlighting. The first, tagged as CRDB v3.0, added $\mathrm{H}$ and He data from a large number of balloon flights going back to the 1950s. These data sets were used to derive Solar modulation levels over sixty years and compared to values derived from neutron monitor data in Ghelfi et al. [1].

The second major upload, tagged CRDB V3.1, was made a few weeks before preparing this release. The update is the result of a tremendous concentrated effort to include a wealth of data released in the last two years from various experiments (AMS-02, CALET, NUCLEON, TRACER, Voyager, and a few others); they correspond to some of the most interesting and high-precision CR data ever recorded. In addition, time-dependent fluxes and ratios from AMS-02 [47,48] and PAMELA [49] were added.

We emphasise at this point that the $\mathrm{CRDB}$ is open for data submissions from experiments since its first release, and contributions are very welcome. We further simplified the submission interface, presented in Section 4.4, to encourage more data submissions in the future.

\subsection{Very Heavy and Ultra-Heavy CRs}

The theory of stellar nucleosynthesis to explain Solar system abundances goes back to the fifties [50]. In the following decade, $Z \geq 30$ [51,52] and even a few $Z>90$ (actinides) [53-55] CRs were detected. The abundance of these UHCRs are driven by three processes, corresponding to three categories of stable heavy nuclides: the $\mathrm{p}-, \mathrm{r}-$, and s-nuclides, respectively located at the neutron-deficient side, neutron-rich side, and bottom of the valley of nuclear stability [56]. How much p- [56,57], r-, and s-processes [58,59] contribute to the various UHCRs, and which astrophysical sites are involved remain debated. Moreover, the abundance of UHCRs slightly differs from the Solar system ones, and these differences are likely to be related to specific acceleration (e.g., [60]) or transport processes (e.g., [61]).

Measuring UHCR data is challenging because the fluxes are low. From $Z=28$ (Fe) to $Z=30$ (Zn) and then $Z=40(\mathrm{Zr})$, the flux is suppressed by a factor $\sim 10^{3}$ and $\sim 10^{5}$ [4]. The rarest CRs, the actinides $Z \geq 90$ (Th and $U$ ), are about seven orders of magnitude less abundant than Fe [62]. Standard techniques used for measurements of $Z<30$ CRs can still be pushed to cover the $30 \leq Z \leq 60$ region [4], but come slightly short for the heaviest ones [63,64]. An alternative consists of passive detectors exposed for long durations (several years). The detection principle is based on the chemical modification made in a solid state nuclear track detector when ionising particles go through it. After long exposure, the detector is etched in a chemical agent and each track left is analysed and its properties can be related to the charge and velocity of the CR.

We added in CRDB V4.0 most of the CR data found for $Z>30$. Most measurements do not resolve elements, and we needed to create new name holders for groups of charges, for instance Pt-group (74 $\leq Z \leq 80)$, Pb-group $(81 \leq Z \leq 87)$, subact inides $(74 \leq Z \leq 87)$, and Actinides $(Z \geq 88)$-see Table A2. We note that in the literature different groups sometimes used slightly different charge range in their definition, but we stick to this one for all implemented data-data uncertainties are larger than the error made from using slightly enlarged or reduced charge groups. The data themselves mostly consist of ratios of elements (or of groups of elements) in an unspecified energy band (related to the detector capabilities). In practice, CR fluxes are expected to be maximal at a few $\mathrm{GeV} / \mathrm{n}$, and several experiments reported no energy evolution (within the uncertainties) of their measured ratios. For this reason, and for simplification, we set all UHCR data $Z>50$ to a generic energy bin around $1.5 \mathrm{GeV} / \mathrm{n}$. 
More than sixty years after their discovery, only very few UHCR data are available and almost no new experiment is running or planned. Except for the relatively recent superTIGER experiment in the $Z=30-40$ range [65], the experiments in the high charge range date back to the end of the seventies (Ariel6 [63], HEAO3-HNE [66,67]), the early eighties (UHCRE-LDEF [3,68,69]), or at best the nineties (Trek $[70,71])$. The most recent results on subactinides and actinides come from indirect measurements of the CRs from their impact on meteorite olivines [72-74], namely the Olimpiya Experiment (not implemented yet in $\mathrm{CRDB}$ ). The solution to the origin of these elements may come from yet another route: recently, optical follow-up observations of a gravitational wave event have shown that binary star mergers could be a major r-process contributor to UHCRs [75]. More such events are likely to be detected in the future, and they will provide a complementary view to that of CR data.

\subsection{Antimatter CRs}

So far, antiprotons are the only antinuclei found in CRs. They were first detected at the end of the seventies [76,77] and soon interpreted by Silk and Srednicki [78] as possible dark matter signatures. The pioneering theoretical aspects and subsequent efforts were acknowledged by the 2019 Cosmology Gruber prize: "Silk recognised dark matter's indirect signatures such as antiprotons in cosmic rays and high energy neutrinos from the Sun". This exploration continues with modern experiments, which have detected several tens of thousands of antiprotons [79].

The quest for $Z<-1 C R s$ is also very much alive but the expected fluxes are very low. Indeed, most if not all CRs antiprotons can be attributed to nuclear production ( $\mathrm{H}$ and $\mathrm{He} C \mathrm{CRs}$ on the interstellar gas) during $\mathrm{CR}$ propagation in the Galaxy. The cost to create and fuse together an extra antiproton or antineutron from colliding protons is a roughly $10^{-4}$ suppression factor (e.g., [80]). As a consequence, the ratio of astrophysical CR antiprotons to protons is at most $\sim 10^{-4}$ (e.g., [79]), and the ratio of astrophysical CR antinuclei (of atomic number $A$ ) to protons is expected to go very roughly as $10^{-4 A}$. So far, only upper limits have been derived on the latter [81]. CR antideuterons are particularly difficult to detect because they must be isotopically separated from the $\bar{p}$ tail, and also because charge confusion rejection from $p$ should be at the level of $10^{8}$. The AMS-02 experiment on the ISS or the GAPS experiment based on a novel detection technique could detect a few events in the near future [11]. Although at a much lower level, antihelium events may have been seen in AMS-02 very preliminary analyses [12]. Whether these events are real, and whether this is compatible with no detection of antideuterons and can be accounted for physics scenario is still at the exploratory stage [82-84]; a confirmation of these events would have huge consequences.

To tackle upper limits, a new key in the CR database table was added (see Section 2.1). Limits on antielements are also usually derived with respect to elements, and we added in CRDB V4.0 new names to cover all associated data (see Table A2). The best limits so far come from the BESS balloon flights [10] and PAMELA mission [85], as shown in Figure 2 with results from other experiments derived over the years (all included in CRDB $\mathrm{V}^{.0}$ ). 

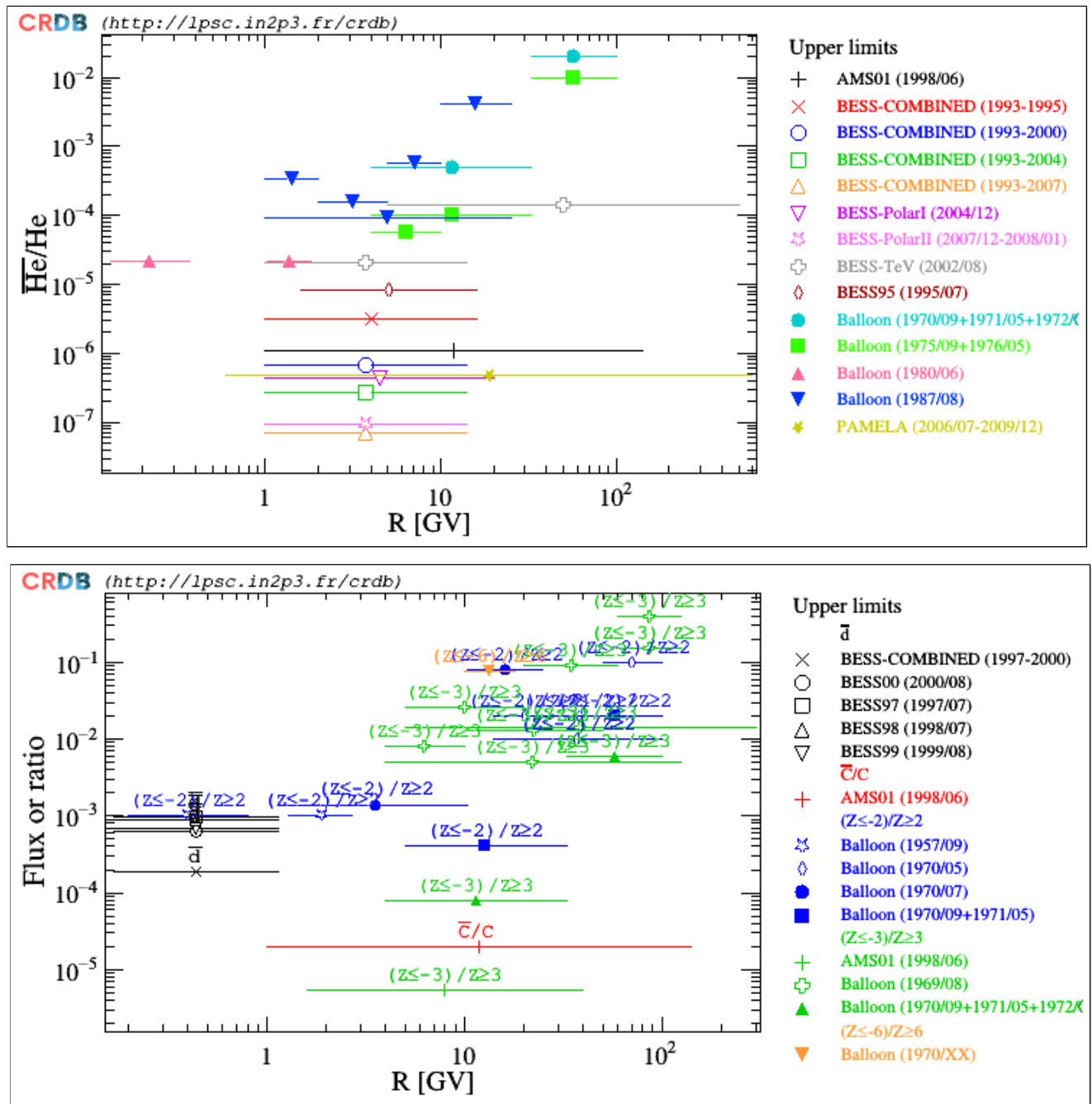

Figure 2. New antinuclear data in $\mathrm{CRDB}$ v 4.0. (Top panel): Upper limits on antihelium-to-helium ratio [10,77,86-94]. (Bottom panel): Upper limits on antideuteron flux [95] (black symbols) and various combinations of antielement-to-element ratios [86,95-103].

\subsection{Ultra-High Energy CRs}

A major extension of the already comprehensive $C R$ database is the inclusion of data from air shower experiments. High-energy CRs are measured indirectly with ground-based experiments. They detect the particle cascades initiated by CRs in Earth's atmosphere (air showers). Since the CR flux drops rapidly with increasing energy, the air-shower technique is the only feasible way to obtain $\mathrm{CR}$ data above PeV energies. The air-showers allow for large aperture experiments due to their extensive footprint on the ground in charged particles and Cherenkov light, and their visibility from a distance in ultra-violet light.

The energy and direction of a CR can be well inferred from air showers, but the identity of each individual CR cannot be determined accurately. The identity, more specifically the mass $A$ of the $\mathrm{CR}$, has to be inferred from air shower properties that naturally fluctuate, like the slant depth $X_{\max }$ of shower maximum measured from the top of the atmosphere or the total number $N_{\mu}$ of muons produced in the shower. These natural fluctuations make it impossible to distinguish species on an event-by-event basis. Therefore, fluxes of individual elements or isotopes cannot be determined.

Experiments most commonly report the all-particle flux. In principle, this also includes stable electromagnetically and weakly interacting particles, but the flux of these components at the $\mathrm{PeV}$ scale and above is negligible compared to nuclei [104,105]. In addition, some experiments report the 
fluxes of mass groups by splitting the observed mass range into two groups (proton-helium group and the rest), four groups (proton, helium, oxygen-, and iron-group), or five (as before, but adding a silicon group). These groups span over roughly equal intervals in $\ln A$, since the mass sensitivity is roughly constant in this variable. Beside the usual individual element or isotope names, the list of $C R$ quantity names in CRDB V4.0 was expanded to handle these groups (e.g., H-He-group, Fe-group, AllParticle, see Table A2).

We note that these results are obtained by simulating air showers initiated by the leading element in each group only, and by fitting the sum of the air shower response distributions to the observed distribution [106,107]. Representing a whole mass-group by a single element is a great simplification, but unavoidable since the relative fluxes of species within a mass group are unknown. The analysis approach works in practice because air shower fluctuations are large compared to the small differences in the response to individual elements within a group.

Another peculiarity of high-energy data is that one raw data set often has several interpretations, in which the fluxes of mass groups and, to a lesser degree, also the all-particle flux vary. These parallel interpretations are reported by the experiments due to significant theoretical uncertainties in simulated air showers. Detailed air shower simulations are required to infer the mass $A$ from air shower properties like $X_{\max }$ and $N_{\mu}$, and these simulations use hadronic interaction models that extrapolate fixed-target and collider measurements of particle interactions using theory and phenomenology. Air showers are dominated by soft QCD (Quantum Chromo Dynamics) interactions, which so far cannot be predicted accurately from first principles. Therefore the interpreted measurements depend on the hadronic interaction model used in air shower simulations, which is listed with the interpreted data.

For this update, we focus on adding flux measurements to the $\mathrm{CRDB}$, but we also consider to include measurements of air shower properties like the average of $X_{\max }$ or $N_{\mu}$ as a function of the shower energy in the future. In the current update, we include air-shower based flux data from the Pierre Auger Observatory, Telescope Array, the IceCube Neutrino Observatory, the KASCADE-Grande experiment, the TUNKA-133 Array, and the H.E.S.S. observatory. An illustration of the evolution with energy of the ratio of two groups of elements is shown in Figure 3.

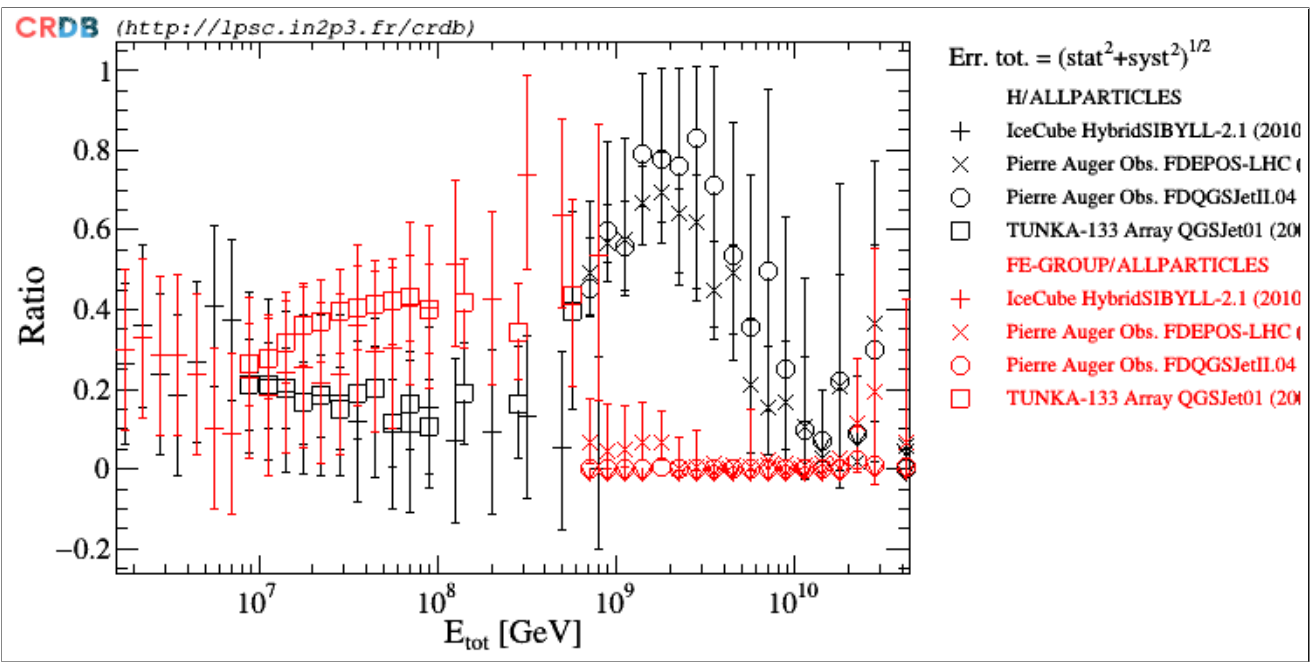

Figure 3. Sample of UHECR (Ultra-high energy cosmic-ray) data added in CRDB v4 . 0. For illustration, we show the fraction of light (H) and heavy (Fe-group) cosmic rays for IceCube [108], Pierre Auger Observatory [109] for two different Monte Carlo event generators, and TUNKA-133 [110]. Note that very long subexp names always get truncated in CRDB displays (as seen in the legend).

\section{User-Interface Updates and New Submission Form}

CRDB is hosted by the LPSC laboratory website. It runs on a LAMP solution, i.e., a stack of free open source softwares: Linux (operating system), Apache HTTP (server), MySQL (database), and PHP 
(hypertext pre-processor language). The web interface relies on several third-party libraries (jquery, jquery-ui, jquery.cluetip, and table-sorter) that are used to sort and display the database content, as briefly presented in the various sections below. For efficiency and speed, all web pages make use of AJAX (Asynchronous JavaScript and XML) web development techniques.

Accessing the data in $C R D B$ can be achieved in two ways, either by accessing the resources via $C R D B$ web pages, or via a REST (representational state transfer) interface. While web pages allow to access the fully contextualised data and meta-data (thanks to several sorting, selections, display, and download of data options), the REST interface is useful for users who want to access and retrieve data from command lines (i.e., from their terminal or codes, without going through the CRDB web pages).

Below, we discuss separately the various web interfaces. We start with the description of the improvements brought on those already available in the first release (Section 4.1). We then describe the interface for extracting $\phi_{\mathrm{FF}}$ time series (Section 4.2), the new help page for the REST interface (Section 4.3), and the simplified procedure (and format) to submit new data (Section 4.4).

\subsection{Web User Interface}

The address https:/ / lpsc.in2p3.fr/crdb leads the user to a choice of various tabs, as illustrated in Figure 4. Among them, two tabs provide differently contextualised informations on CR experiments and data, while other tabs provide general information on $\mathrm{CRDB}$, external CR resources, etc.

We give below a brief description of these tabs, highlighting the most salient features and novelties of this version, and providing a few snapshots as illustrations.

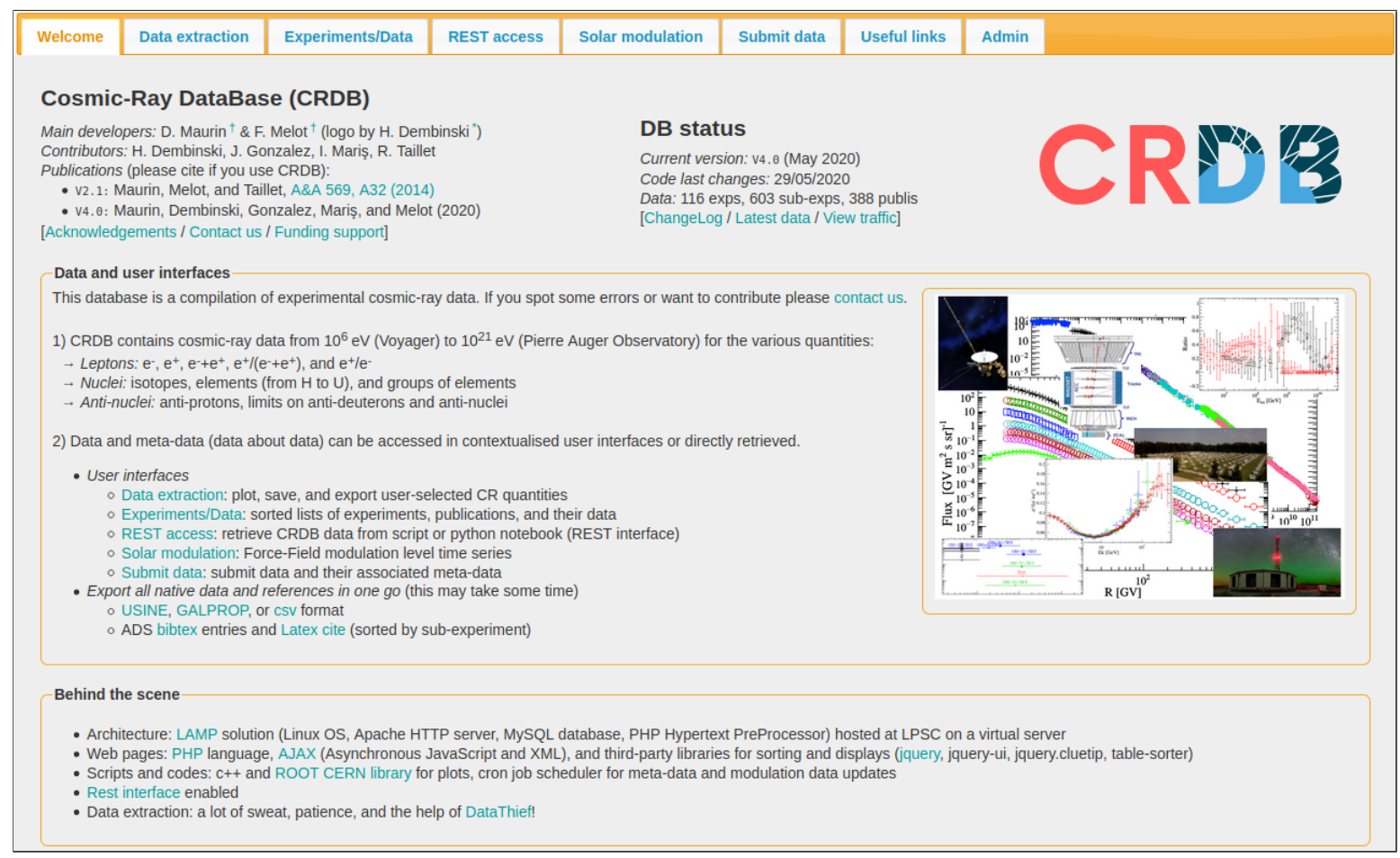

Figure 4. Snapshot of the 'Welcome' tab to which users are directed from the CRDB webpage https: / / lpsc.in2p3.fr/crdb. Clicking on the tabs (shown on top) allows to access the various user-interfaces presenting in different ways the contextualised data and meta-data (see text).

\subsection{1. 'Welcome' Tab}

This is the unique entry point of the website (see Figure 4). It contains a brief description of the database content and structure, and informations on CRDB including versions, $\log , \log$, 
and publications. In particular, the log file tracks the changes made in the various releases (data corrections and past and ongoing developments).

\subsection{2. 'Experiments/Data' Tab}

This is the tab to go for users looking for a specific experiment, its meta-data, and the data it gathered; experiments can be sorted according to their name, starting date, or type. This tab lists the quantities they measured. It also provides links to the experiment web page, the associated publications, and pictures of the sub-detectors (by clicking on the 'magnifying glass' icon); see Figure 5 for an illustration. Clicking on '[data]' for any given sub-exp gives further information on the CR data origin: data taking period, how data were retrieved (numbers from tables, extracted from figures, or from personal communications), details on the detector, etc.

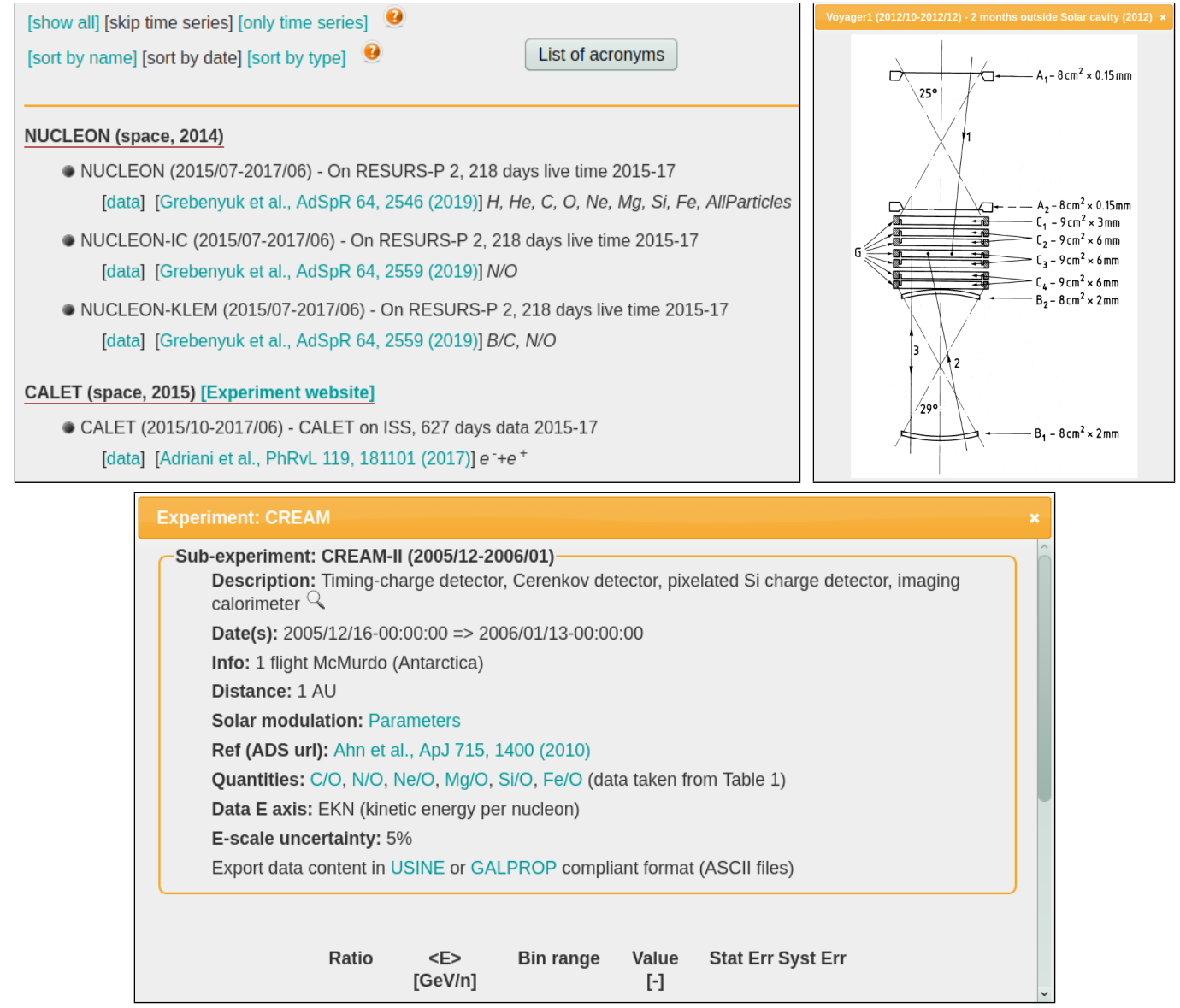

Figure 5. Snapshot from the EXPERIMENTS/DATA tab illustrating how experiments, sub-experiments, and publication meta-data are organised in this tab (top left panel). From here, clicking on (i) the 'List of acronyms' button provides the meaning of acronyms for all experiments in CRDB (not shown), (ii) the magnifying glass icon (seen beside sub-experiment names) pops-up the detector schematics (top right panel) showing Voyager in this example, (iii) the '[data]' link pops-up the full meta-data informations and associated data (bottom panel).

We stress that the data presented in this tab are 'native' data only (see Appendix A.1), i.e., data as provided in publications and uploaded in the database without modification (which is not the case in the 'Data extraction' tab). Accordingly, the energy axes for the data are as provided in the publications. 


\subsection{3. 'Data Extraction' Tab}

This is the main interface to select, extract and plot data, displaying also some meta-data (sub-exp names, links and BIBTEX references for publications matching the selection), with the possibility to save the outputs in different formats (images, ASCII files, etc.). The web page consists of a selection box allowing to choose a CR quantity (numerator and denominator) and to display all native and combined data (see Appendix A.1) ${ }^{16}$ _for caution about the usage of overlapping data taking periods for data from the same experiment, we refer the reader to Appendix A.5. More selection criteria allow to ask for matching sub-exp (partial or full) names, specific dates and energy range, and flux rescaling with energy. In the context of long time series provided by both the AMS-02 [47,48,111] and PAMELA [49] experiments, CRDB V4. 0 allows to select only (or discard) data from time series, in order not to overcrowd the display of data. This is illustrated on a 'time-series only' selection in Figure 6, where we show Carrington rotation-averaged PAMELA H data from 2006 to 2010 [112]. This list triggers on the presence of the word 'average' (in yearly, monthly, daily, Bartels rotation... averages) in the 'subexp-info' key in the SUBEXP table (see Section 2.2.2).

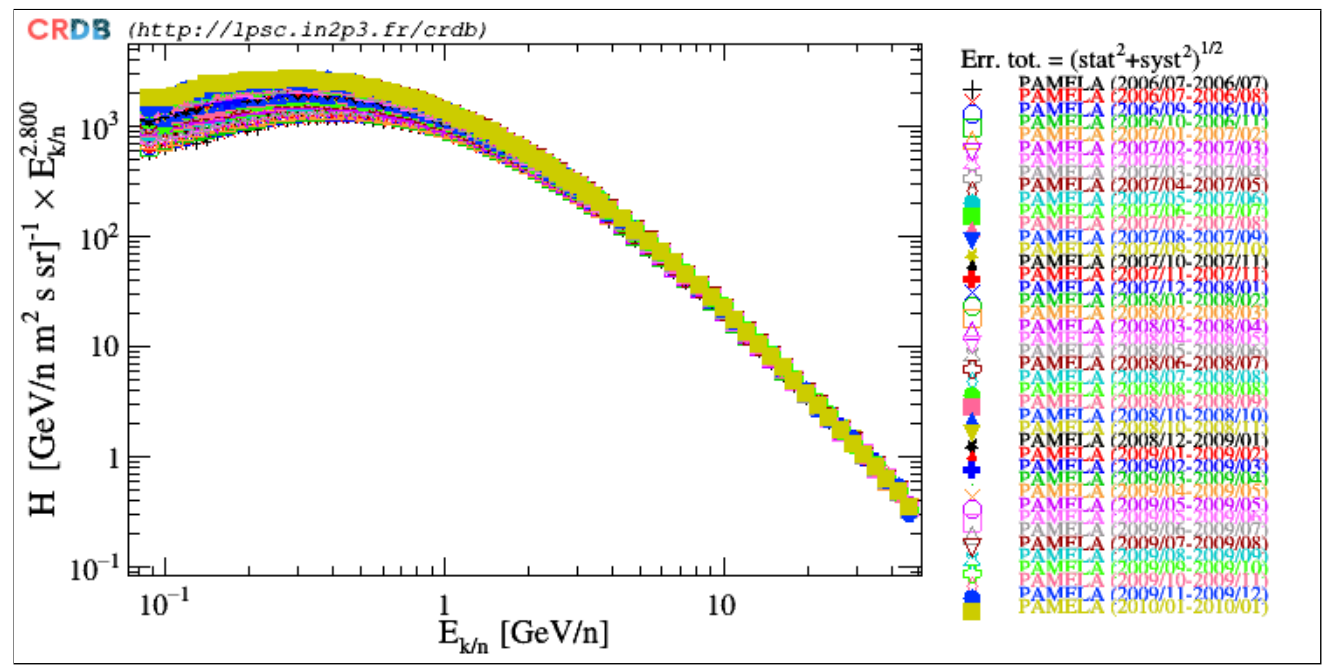

Figure 6. Illustration of the 'time series only' option in the query selection criterion on PAMELA data [112].

A critical choice is that of the energy axis (among $E_{k / n}, R, E_{\text {tot, }}$ etc.): if no native data exist for this energy axis, conversion are performed to rescale the data from its native axis to the queried one. However, this conversion is only possible for fluxes (not ratios), and is exact for CR isotopes and leptons (whose $A, Z$, and $m$ are identified) but approximate for elements and group of elements (see Appendix A.4).

Hitting the 'Extract Selection' button pops-up a new window displaying: (i) a plot of the data, (ii) list of meta-data (per sub-exp) associated with the data, (iii) a summary of the extraction process (i.e., whether combinations and approximations were made), and (iv) a full listing of all retrieved data points (energy, values, and uncertainties). In CRDB $v 4.0$, we improved the browsability between these informations. From the plot, we can export the data in a format compliant with the US INE and GALPROP propagation code, or as a cVs or tarball of ASCI I files. We can also directly retrieve images or ROOT ${ }^{17}$-compliant macros (.root, . C). A 'replot' button allows to further trim the data and modify

16 Publications cannot provide all combinations of their data: for instance, if $n$ fluxes are measured, $n \times(n-1) / 2$ independent ratios can be formed, which can be a large number. For this reason, when extracting data, the query looks first among native data, and if none is found, then for possible combinations leading to the desired quantity.

17 http://root.cern.ch. 
the plot axes, look, and error bars shown, with the same options (as in the original plot) to export and save the results.

To conclude on this tab, we are also pleased to provide in CRDB $v 4.0$ an additional selection option, that allows to display a comma-separated list of quantities: we stress that all selection criteria (specific energy range, dates, sub-experiments, etc.) are enforced to all quantities in the list. An illustration is provided Figure 7, where we show the full CR spectrum from $\mathrm{MeV}$ to EeV energies from a selection of recent experiments (top panel), and a selection of all nuclear fluxes from the AMS-02 experiment (bottom panel).
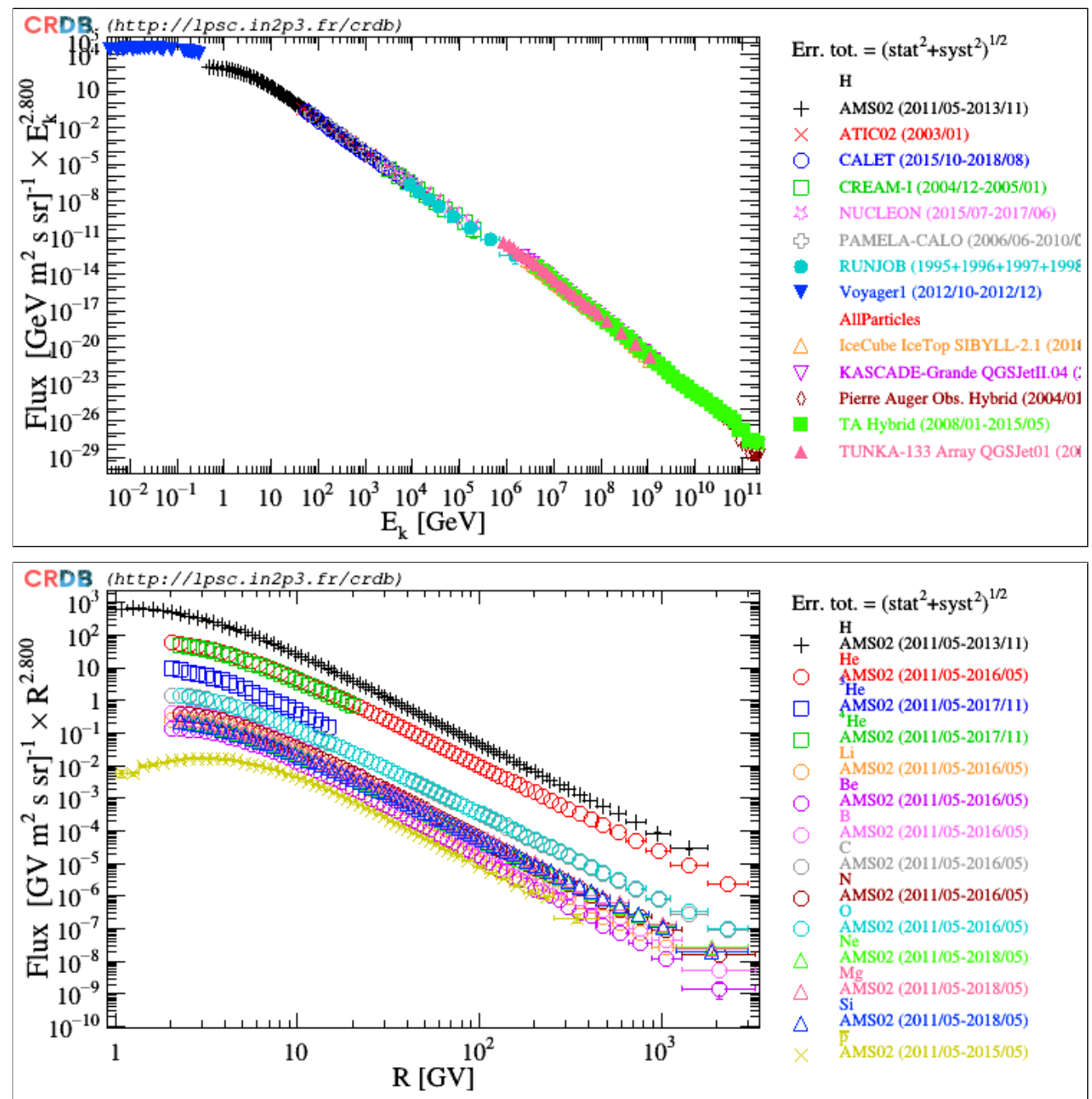

Figure 7. Illustration of the new multi-quantity plotting capability in CRDB $v 4.0$ : (Top panel): H flux from IS MeV data (Voyager1 [113]) up to PeV data (AMS02 [13], ATIC02 [114], CALET [115], CREAM-I [116], NUCLEON [117], RUNJOB [118], PAMELA-CALO [119]); and AllParticle flux from PeV to EeV energies (KASCADE-Grande [120], IceCube IceTop [108], and Pierre Auger Observatory [121]), Telescope Array [122], and TUNKA-133 Array [110]). (Bottom panel): Nuclear component measured to date by AMS-02 [13,15,79,111,123-125]. 


\subsection{4. 'Admin' Tabs}

This tab can be accessed by authenticated users only (CRDB maintainers). In this page, various scripts provide internal checks of the database content (missing images for the detectors, orphan ID in the various tables, list of superseded publications, etc.). This page used to handle validation forms for submitted data, but they were removed thanks to the new submission form in CRDB V4. 0 (see Section 4.4). We can also monitor from this page the traffic on CRDB, stored in a specific LOG_QUERIES table (connection date, IP address, and page visited, see Figure 1). This traffic since August 2013 is shown in Figure 8. CRDB received more than a quarter million queries, from $\sim 20,000$ different IP addresses in over a hundred countries-most queries originate from Germany, USA, Italy, France, China, Switzerland, and Japan, i.e., countries strongly involved in recent experiments and phenomenology.

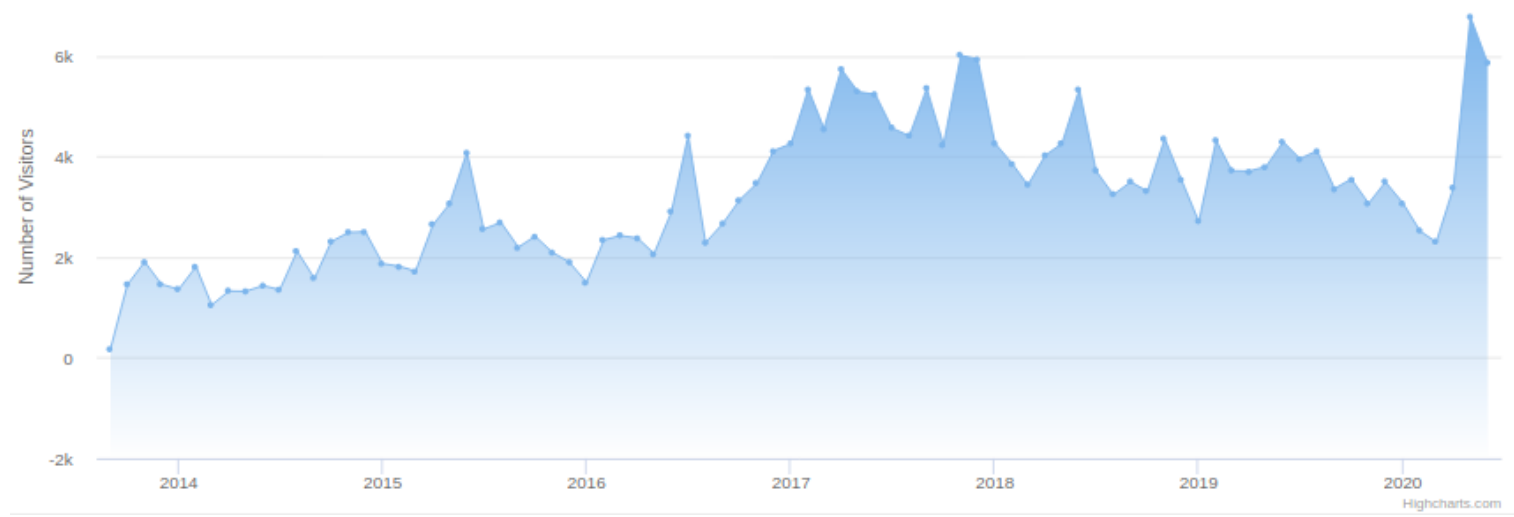

Figure 8. Statistics of monthly CRDB connections since August 2013. The peak in May 2020 is related to queries made for systematic checks before the release of $\mathrm{CRDB} \vee 4.0$; to a lesser extent, a similar peak is visible in August 2016 related to the release of CRDB v3.0. In CRDB V4 .0, this traffic plot is now accessible for any user via a link in the 'Welcome' tab.

\subsection{5. 'Useful Links' Tab}

This tab gathers links to many online CR resources. These links include propagation codes for Solar, Galactic and extra-galactic CRs, other useful CR databases, and CR-related websites. If you feel that some important resources are missing, please contact us (crdb@lpsc.in $2 \mathrm{p} 3 . \mathrm{fr}$ ), we will be happy to add them.

\section{2. 'Solar Modulation' Tab}

This tab was added in CRDB v3.0 and thus not described in Maurin et al. [22]. Its purpose is to provide Solar modulation level $\phi_{\mathrm{FF}}$ in the Force-Field approximation (e.g., [31]) for any date between the 1950s and today. The calculation is based on neutron monitor (NM) data and the reconstruction algorithm of Ghelfi et al. [1].

Neutron monitors are devices developed in the 1950s to monitor Solar activity [126]. They combine a very good time resolution (a few minutes) and a good stability over decades. For this reason, they are well-suited to get time series over large time periods [37]. These values are for instance used to fill $\phi_{F F}$ from any CRDB sub-experiment as discussed in Section 2.2.2. Averaged $\phi(t)$ time series (bottom panel) can also be retrieved, as illustrated in Figure 9, from a selection (top panel) of NM stations, a time period, and a time resolution (from the finer-grain $10 \mathrm{~min}$ to monthly averaged time series). 


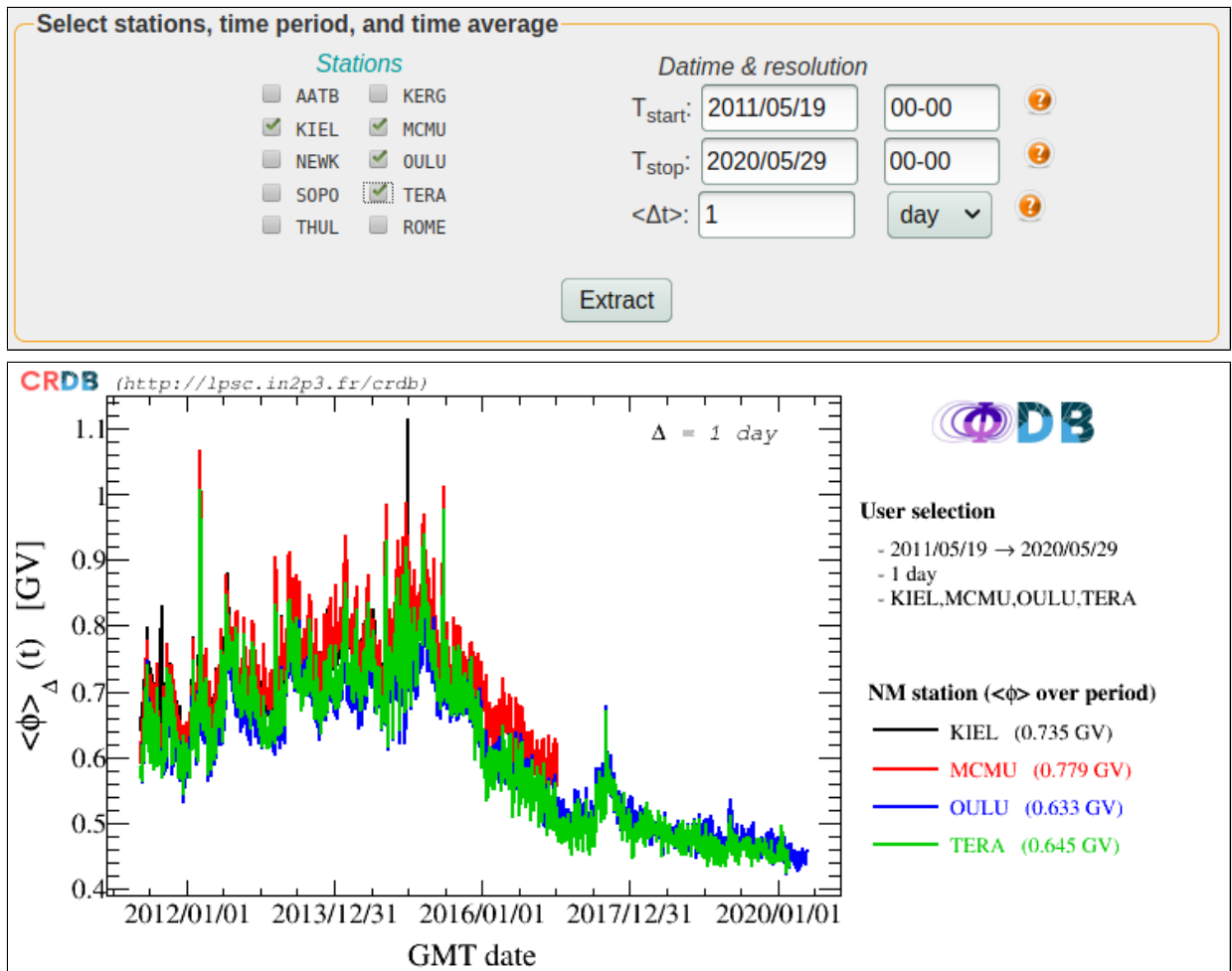

Figure 9. Illustration of $\phi$ time series retrieved from the ' $\phi$ from $\mathrm{NM}^{\prime}$ tab, based on the calculation of Ghelfi et al. [1]. The top panel shows the user-selection for NM stations (OULU, KIEL, MCMU, and TERA) and time interval 2011/05 to today, corresponding to the time since AMS-02 was installed on the ISS. The bottom panel shows the resulting daily averaged values $\langle\phi\rangle_{d}(t)$ as coloured lines for different stations.

In practice, scripts on LPSC servers retrieve and process NM data from NMDB. They produce, behind the scene, ASCI I files for all available stations at a $10 \mathrm{~min}$ time resolution. Then for each CRDB query, another layer of scripts reads these files to calculate and display the user selection. To ensure a continuous service and update, $\mathrm{a} c r o \mathrm{n}^{18}$ time-based job scheduler is used on a daily basis.

\section{3. 'REST Access' Tab}

The database offers a REST ${ }^{19}$ interface, which makes it easy to download data sets according to selection criteria from another program. The interface has been available since version CRDB V1.3, but the new help page in CRDB $v 4.0$ makes this feature more prominent.

Data sets can be selected by quantity (element, isotope, electron or positron, or mass group), experiment, energy range, and time range. Users must specify the energy type in their query. As mentioned in previous sections, data sets are stored in their native energy type, which can be kinetic energy, kinetic energy per nucleon, total energy, rigidity. The native type is converted to match the request, and details on the conversion are given in Appendix A.4. Other parameters of the interface control the output format, and whether ratios should be synthesised from individual flux measurements.

The available parameters are documented in Table 1 (also reproduced on the website). To give an example, the boron-to-carbon flux ratio as a function of the kinetic energy per nucleon can be queried with the command-line utility curl as follows:

18 https://en.wikipedia.org/wiki/Cron.

19 https://en.wikipedia.org/wiki/Representational_state_transfer. 
curl -L'http://lpsc.in2p3.fr/crdb/rest.php?num=B\&den=C\&energy_type

$=\mathrm{EKN}^{\prime}>\mathrm{db} \cdot \mathrm{dat}$

The output in USINE format (table with columns separated by spaces) is stored in the file db . dat. Queries can be made from any general purpose programming language. For Python users, we provide a simple module to run queries from Python and return the result as a numpy array. The code and a tutorial with example usage are available on $\mathrm{GitHub}^{20}$ and linked to from the CRDB website.

Table 1. REST interface parameters, description, and examples values. Only the first three parameters are mandatory. See text for how to use REST in a command line.

\begin{tabular}{|c|c|c|}
\hline Parameter & Description & Allowed Values (Examples) [Default] \\
\hline \multicolumn{3}{|c|}{ Native data selection (mandatory) } \\
\hline num $^{a}$ & $\begin{array}{l}\text { Numerator of quantity to retrieve (element, } \\
\text { isotope, lepton, mass group) }\end{array}$ & CRDB name (H, 1H-BAR, e-, O-group) \\
\hline $\operatorname{den}^{a}$ & $\begin{array}{l}\text { Denominator of quantity to retrieve (only } \\
\text { for ratio, leave empty for flux) }\end{array}$ & $\begin{array}{lccc}\text { CRDB name } & \left(\mathrm{He}, \quad 1 \mathrm{H}, \mathrm{e}^{-+\mathrm{e}+}\right. \\
\text { AllParticles }) & & & \\
\end{array}$ \\
\hline energy_type & $\begin{array}{l}\text { Energy axis for returned quantities: kinetic } \\
\text { energy per nucleon }(\mathrm{GeV} / \mathrm{n}) \text {, kinetic } \\
\text { energy }(\mathrm{GeV}) \text {, rigidity }(\mathrm{GV}) \text {, total energy } \\
(\mathrm{GeV}) \text {, or total energy per nucleon }(\mathrm{GeV} / \mathrm{A})\end{array}$ & CRDB keyword (EKN, EK, R, ETOT, ETOTN) \\
\hline \multicolumn{3}{|c|}{ Data combinations and/or modifications (optional) } \\
\hline combo_level ${ }^{b}$ & $\begin{array}{l}\text { Add combinations via ratio or product } \\
\text { of native data (from the same sub-exp at } \\
\text { the same energy) that match quantities in } \\
\text { list (e.g., compute B/C from native B } \\
\text { and C). Three levels of combos are enabled: } \\
0 \text { (native data only, no combo), } 1 \text { (exact } \\
\text { combos), or } 2 \text { (exact and approximate } \\
\text { combos): in level 1, the mean energy (or } \\
\text { energy bin) of the too quantities must be } \\
\text { within } 5 \% \text {, whereas for level } 2 \text {, it must be } \\
\text { within } 20 \%\end{array}$ & Integer in $0-2(0,1,2)[1]$ \\
\hline energy_convert_level ${ }^{c}$ & $\begin{array}{l}\text { Add data obtained from an exact or } \\
\text { approximate energy_type conversion } \\
\text { (from native to queried). Three levels of } \\
\text { conversion are enabled: o (native data } \\
\text { only, no conversion), } 1 \text { (exact conversion } \\
\text { only, which applies to isotopic and leptonic } \\
\text { fluxes), and } 2 \text { (exact and approximate } \\
\text { conversions, the latter applying to flux of } \\
\text { elements and of groups of elements) }\end{array}$ & Integer in $0-2(0,1,2)[1]$ \\
\hline flux_rescaling & Multiply fluxes by $\langle E\rangle^{\text {flux_rescaling }}$ & Decimal number $(1,2.5)[0]$. \\
\hline
\end{tabular}

20 https://github.com/crdb-project/tutorial. 
Table 1. Cont

\begin{tabular}{|c|c|c|}
\hline Parameter & Description & Allowed Values (Examples) [Default] \\
\hline \multicolumn{3}{|c|}{ Trimming the selection (optional) } \\
\hline energy_start & Lower limit for energy_type & Floating point number $(2 \mathrm{e}-1,1.2 \mathrm{e} 2)[1 . \mathrm{e}-40]$ \\
\hline energy_stop & Upper limit for energy_type & Floating point number $(1 \mathrm{e} 5,5.3 \mathrm{e} 7)$ [1.e40] \\
\hline time_start & Lower limit for interval selection & $\begin{array}{l}\text { Date YYYY or YYYY / MM } \\
(2014,2010 / 06)[1950]\end{array}$ \\
\hline time_stop & Upper limit for interval selection & $\begin{array}{l}\text { Date YYYY or YYYY / MM } \\
(2020,2019 / 06)[2050]\end{array}$ \\
\hline time_series & $\begin{array}{l}\text { Whether to discard, select only, or keep } \\
\text { time series data in query }\end{array}$ & CRDB keyword (no, only, all) [no] \\
\hline exp_dates & $\begin{array}{l}\text { Comma-separated list (optional time } \\
\text { intervals) of sub-experiment partial or full } \\
\text { names }\end{array}$ & $\begin{array}{l}\text { Partial name (AMS, ACE), full name } \\
\text { (BESSPolarI), or combinations of names } \\
\text { and time intervals (PAMELA }(2006: 2008) \text {, } \\
\text { BESS }(2002 / 01: 2004 / 12))[\mathrm{n} / \mathrm{a}]\end{array}$ \\
\hline \multicolumn{3}{|c|}{ Outputs (optional) } \\
\hline format & File output format & $\begin{array}{l}\text { CRDB keyword (CSV, usine, galprop) } \\
\text { [CSV] }\end{array}$ \\
\hline modulation & Source of Solar modulation values & $\begin{array}{l}\text { CRDB keyword (USO05, USO17, GHE17) } \\
\text { [GHE17] }\end{array}$ \\
\hline
\end{tabular}

${ }^{a}$ Url syntax requires eะ2B instead of e+ for a positron. ${ }^{b}$ See Appendix A.1 for a discussion on how to form combos and propagate errors from the native data. ${ }^{c}$ Energy type conversions are exact for isotopic and leptonic fluxes, but not for elements, since an elemental flux in general is the sum of several isotope fluxes in often unknown fractions. Usually, isotope fractions have to be assumed (see Appendix A.4).

\section{4. 'Submit Data' Tab: New Submission Form}

In CRDB V2.1, the submit data interface was based on a sequential online 4-step procedure. The user had to fill meta-data first (experiment, sub-experiment, and then publication) and the data. Each step had to be completed and validated by one of us (via the admin interface) before being able to go to the next step. In practice, because of the too many steps, probably not documented enough procedure, and delay between the validation steps, this was almost never used.

In $C R D B \vee 4.0$, we decided to completely change the approach and only ask for a single Csv file, whose expected columns are described in Table 2 (and also reported on the 'Submit data' tab); files must be sent to $\mathrm{crdb}$ Ipsc.in $2 \mathrm{p} 3$. fr. One of us then uses dedicated scripts on these files to finalise the upload on CRDB. In this new format, informations on the data and meta-data are still required, but to simplify the submitter task, many entries can be left empty (and will be filled by us), as indicated by stars in Table 2. We hope that this simplified submission procedure and lessened number of meta-data entry to provide will convince experimentalist to make the extra step of submitting the data to CRDB after they submit their publication to a journal. 
Table 2. Description of the 23 expected columns in the CSV file prepared by the user to submit data. The table shows the column ID, the associated keyword in the database structure (see Figure 1), the expected content of the column, and an example (and associated unit) for this column — entries corresponding to starred keywords * can be left empty (i.e., " ") if the user is unsure. For more details on CRDB data and meta-data definitions, see Section 2.1 (data points), Section 2.2.1 (experiments), Section 2.2.2 (sub-experiments), and Section 2.3.1 (publications).

\begin{tabular}{|c|c|c|c|}
\hline Col. & (Meta-)Data & Description & "Example" [Unit] \\
\hline 1 & EXP-NAME & Experiment name & "AMS02" \\
\hline 2 & EXP-TYPE & balloon, ground, or space & "space" \\
\hline 4 & EXP-STARTYEAR * & Experiment starting year & 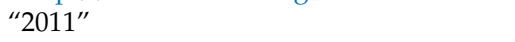 \\
\hline 5 & SUBEXP-NAME * & Name specific to analysis (Section 2.2.2) & "AMS02 (2011/05-2012/12)" \\
\hline 6 & SUBEXP-DESCRIPTION * & Detector information & "9 planes of silicon tracker, TRD, etc" \\
\hline 9 & SUBEXP-DISTANCE & Sub-exp. average distance to Sun & "1" [AU] \\
\hline 10 & SUBEXP-DATES & Start-stop data taking period & “2011/05/19-000000:2012/12/10-000000" \\
\hline 11 & PUBLI-HTML & Publication ADS ID or doi name & “2013PhRvL.110n1102A” \\
\hline 12 & PUBLI-DATAORIGIN * & Source for the data in the publication & “Table 1, Figures 2 and 3" \\
\hline 13 & $\operatorname{DATA}_{\mathrm{QTY}}{ }^{\dagger}$ & Measured quantity (CRDB names) & "B/C" \\
\hline 17 & DATA-E_BIN_U & $E_{\text {up }}$ in unit of [DATA-EAXIS] & “5.37” [GV] \\
\hline 18 & DATA-VAL & $y$, measured value & “0.3214" $\left[\mathrm{m}^{-2} \mathrm{~s}^{-1} \mathrm{sr}^{-1} \mathrm{GV}^{-1}\right]$ \\
\hline 19 & DATA-VAL_ERRSTAT_L & $\Delta y_{\text {lo }}$, lower stat. err. & $"-0.0011^{\prime \prime}\left[\mathrm{m}^{-2} \mathrm{~s}^{-1} \mathrm{sr}^{-1} \mathrm{GV}^{-1}\right]$ \\
\hline 20 & DATA-VAL_ERRSTAT_U & $\Delta y_{\text {up }}$, upper stat. err. & $"+0.0011^{\prime \prime}\left[\mathrm{m}^{-2} \mathrm{~s}^{-1} \mathrm{sr}^{-1} \mathrm{GV}^{-1}\right]$ \\
\hline 21 & DATA-VAL_ERRSYST_L & $\Delta y_{\text {lo }}$, lower syst. err. & $"-0.075^{\prime \prime}\left[\mathrm{m}^{-2} \mathrm{~s}^{-1} \mathrm{sr}^{-1} \mathrm{GV}^{-1}\right]$ \\
\hline 22 & DATA-VAL_ERRSYST_U & $\Delta y_{\text {up }}$, upper syst. err. & $"+0.0089 "\left[\mathrm{~m}^{-2} \mathrm{~s}^{-1} \mathrm{sr}^{-1} \mathrm{GV}^{-1}\right]$ \\
\hline 23 & DATA-ISUPPERLIM & " 0 " (measured) or " 1 " (upper limit) & “0” \\
\hline
\end{tabular}

${ }^{\star}$ Columns that can be left empty (but not omitted) if the user is unsure about what he should write. ${ }^{\dagger}$ To help pick the correct denomination for the measured quantities, we list in the webpage all currently defined names. If necessary, quantities not defined yet will be added along with the submitted data.

\section{Summary and Future Developments}

In this article, we have presented $C R D B V 4.0$, a new version of the CR database for charged species, highlighting several improvements on the database structure and content, and on the user interfaces. We summarise the changes made below:

- Database structure: a few tables were simplified and three became deprecated (Solar modulation table from the publication, users and validation tables for the old data submission interface). The pre-defined key values were also modified for the experiment type (now balloon, ground, or space) and energy type (now also includes ETOTN). Two new keys were added in the in the SUBEX_PUBLI and PUBLI tables respectively, in order to keep track of the data origin (retrieved from from a table or figure in the publication, etc.), and to store a possible energy scale uncertainty in the data (single number for a given sub-experiment).

- Database content: this version is associated with a massive upload of CR data. For CRDB V3.1, released a couple of weeks before this version, we filled important GCR high-precision data published in the last two years (AMS-02, DAMPE, NUCLEON, TRACER, Voyager, etc.). For CRDB V4.0, we extended the CR data content towards higher energies (KASCADE-GRANDE, Pierre Auger Observatory, etc.), higher charges Z > 30 (LDEF UHCRE, TREK, etc.), and lower charges $Z<-1$ (upper limits from AMS-01, BESS, PAMELA, etc.). The database now contains more than 100 experiments and 350 publications, covering 14 decades in energy (from $10^{6}$ to $\left.10^{20} \mathrm{eV}\right)$.

- User interfaces: the many improvements we implemented provide users with more options to retrieve data, along with a clearer presentation of the various interfaces. These changes include: 
(i) more data selection options (e.g., on times series) and plotting/export options (.pdf, .csv), including the possibility to display several CR quantities on the same plot; (ii) new help page and an example python notebook script to retrieve data via the REST interface; (iii) updated interface to retrieve Solar modulation level $\phi_{\mathrm{FF}}$ time series (or average) from any time period since $1950 \mathrm{with}$ its dedicated REST interface; (iv) simplified file format to submit new data; (v) extended list of links for online CR resources. We hope in particular that the new submission format, with clearer explanations about the data and meta-data to fill, will help us always keeping CRDB up-to-date in the future.

\section{Final thoughts on CRDB Evolution}

CRDB was thought from the start as a service to the CR community. In an ideal world, we would like experimentalists to submit their data to $\mathrm{CRDB}$ as soon as their results appear on the arXiv preprint server $^{21}$ or in a peer-review journal.

There are several possible directions along which CRDB could be improved. On a technical aspect, we are planning to make the website responsive for a better rendering on diverse electronic devices. On the data content aspect, we will obviously continue to upload new data as they appear, as quickly as we can. We would also be grateful for any help to extend the datasets to new quantities. Indeed, without any change in the database structure, we could quite easily include (i) more relevant quantities for UHECRs, i.e., the mean logarithmic mass $\langle\ln A\rangle$, the mean depth of shower maximum $\left\langle X_{\max }\right\rangle$, etc.; (ii) dipole anisotropy spectral data as presented in Ahlers and Mertsch [127], (iii) upper limits or data on the neutrino CR spectrum discovered by the IceCube Collaboration [128]. As a first step in this direction, near the completion of this article, a preliminary agreement was reached with the KCDC team [24] to include their data in a forthcoming CRDB release. Finally, we could also imagine CRDB as a portal to gather more CR-related data, for instance hosting the many nuclear cross-section data presented in Génolini et al. [129]. These future developments will depend on the workforce available and feedback from the CR community.

To conclude, any help and feedback to further expand the database is welcome. Comments, questions, suggestions, and corrections are to be sent at crdbelpsc.in $2 \mathrm{p} 3 . \mathrm{fr}$.

Author Contributions: Conceptualisation, D.M., H.P.D., J.G., and I.C.M.; methodology, D.M. and H.P.D.; software, F.M. and D.M.; writing—original draft preparation, D.M. and H.P.D.; writing—review and editing, D.M., H.P.D., J.G., I.C.M.; supervision, D.M. All authors have read and agreed to the published version of the manuscript.

Funding: This work was supported by the Programme National des Hautes Energies of CNRS/INSU with INP and IN2P3, co-funded by CEA and CNES.

Acknowledgments: We warmly thank L. Baldini, F. Barao, M. Boudaud, B. Coste, C. Deil, T. Delahaye, L. Derome, F. Donato, M. Duranti, K. Egberts, C. Evoli, H. Gast, J. Gieseler, A. Gil-Swiderska, F. Giovacchini, I. Grenier, S. Haino, G. Jóhannesson, A. Karelin, J. Lavalle, M. Orcinha, M. Paniccia, A. Panov, A. Putze, T. Räihä, B. Shan, A. Strong, L. Sujie, M. Vecchi, A. Vincent, P. von Doetinchem, W. Xu, and S. Zimmer for their feedback that helped correct data in CRDB. D.M. thanks C. Combet for a careful reading of the manuscript, and K. Lockhart for her help on the use of the ADS API. This research has made use of NASA's Astrophysics Data System Bibliographic Services.

Conflicts of Interest: The authors declare no conflict of interest.

\section{Appendix A. Tips and Caveats on Data and Their Extraction}

CRDB users go to the online 'Data extraction' tab or use the REST interface to retrieve data, which is fine. However, they generally overlook how the extraction was made and miss possible caveats with some of the data. Below, we go through a few important items that users should keep in mind. We stress that the REST interface goes through exactly the same steps to extract data from CRDB and thus the same cautions apply.

21 https://arxiv.org/. 


\section{Appendix A.1. Difference between 'Native' and 'Combined' Data}

We recall the core data of $\mathrm{CRDB}$ are 'native' only, i.e., measurements made available in the publications (in tables or extracted from figures ${ }^{22}$ using DATATHIEF III $^{23}$ ). However, many useful combinations can be formed from native data. For instance, starting from $\mathrm{C}$ and $\mathrm{O}$ native fluxes in $\mathrm{CRDB}$, the user can extract $\mathrm{C} / \mathrm{O}$. In order to get meaningful results, this procedure has to ensure that $\mathrm{C}$ and $\mathrm{O}$ are from the same energy range or use the same central energy point. As described in details in Appendix A of Maurin et al. [22], priority rules exist to form such ratios, and tick boxes in the 'Data extraction' interface enable to select the energy tolerance for which CRDB accepts to form the ratios, in order to have exact (energy point within $5 \%$ ) or approximate (energy point between [5-20\%]) combinations.

\section{Appendix A.2. Data Uncertainties in $\mathrm{CRDB}$}

Most pre-1980s experiments were sensitivity-limited, meaning that the error budget was dominated by statistical uncertainties. When retrieved from $C R D B$, these data only show statistical errors and no systematics.

With improved detectors, the practice in the literature shifted towards paying more and more attention to systematic uncertainties. The latter are of various origins (inefficiencies, fragmentation in the detector, etc.). They are either provided as raw text description in the publication (see the example of HEAO-3 data discussed in Appendix A.2 of [22]), or as a single number per data point in tables (separately from the statistical uncertainties). In the latter case, CRDB can directly provide statistical and systematics uncertainties as given in the publication. In the former case, we use the text description to estimate the systematic uncertainties, combining quadratically the various sources of systematics. In case in which the systematics description is not clear enough, or when data are indirectly retrieved (i.e., taken from plots), all the uncertainties are assumed to be of statistical origin ${ }^{24}$.

With the advent of high-precision experiments (e.g., AMS-02), the situation has become even trickier. It is now routine in publications to have tables with statistical and several systematics separately. Furthermore, as highlighted in Derome et al. [26], systematics uncertainties usually are likely to be correlated for nearby energy bins. We have not yet thought of modifications in CRDB to handle more than one systematics in the extraction, even less accounting for covariance matrices of systematics. Until further notice, all systematics are combined quadratically and provided as a single number per data in $\mathrm{CRDB}$.

\section{Appendix A.3. Energy-Scale Uncertainty}

Energy measurements of CR detectors suffer from two types of distortions, random and systematic. The random distortions differ from event to event and average to zero over many events. In flux measurements, these types of distortions lead to a softening of the measured flux compared to the true flux. Correcting these distortions is possible with unfolding methods, which is the responsibility of each experiment. In the following we discuss the second kind of uncertainty. The second type of distortions are systematic and originate from the residual uncertainty of the energy calibration of the

22 In the former case, the data match exactly those provided by the experimental teams, but in the latter case, the manual procedure may lead to data points (energy, central value, and uncertainties) that may deviate from the intended ones. This is especially the case for data shown with large symbols or for fluxes covering several decades in logarithmic scale. However, the precision on the data and uncertainty values obtained via the manual extraction is much better than the size of the data uncertainty (for most data extracted), so that this additional deviation is not an issue. Obviously, if a specific data set obtained by this procedure is crucial for a user's analysis, the user is encouraged to check on the original figure in the publication.

23 http://datathief.org.

24 In principle, statistical errors should be symmetric, but when they include sub-dominant systematics or when data are manually retrieved from plots, asymmetric error bars are obtained (which are filled as statistical uncertainties in CRDB). 
detector. These distortions are the same for each event, do not average out, and lead to shifts in the measured flux compared to the true flux.

In general, the energy distortion itself varies with the (true) energy, but experiments are designed and controlled to keep the relative calibration of energies within the covered energy range to higher accuracy. Therefore, the uncertainty can be usually summarised by a single number, the relative energy-scale uncertainty, for each experiment. Since the CR fluxes are mostly steeply falling power laws $\left(\propto E^{-3}\right)$, even a small energy-scale uncertainty has a significant impact on the uncertainty on the flux. An uncertainty of $10 \%$ in the energy scale translates to a $30 \%$ uncertainty on the flux.

In practice, experiments either include this as a systematic uncertainty for each data point, or quote the energy-scale uncertainty separately (and not propagate it to the flux). This is especially common for air shower experiments, which have energy-scale uncertainties of $10 \%$ to $24 \%$. Whenever available in the publications, we fed this number to $\mathrm{CRDB}$, so that it can be retrieved by users. However, we stress out that this uncertainty is never accounted for in CRDB displays.

\section{Appendix A.4. Energy Axes and Conversion to Other Energy Axes}

Most GCR data in CRDB are in kinetic energy per nucleon, as it was the standard for a long time. However, from an instrumental point of view, spectrometers measure rigidities and calorimeters the total energy, etc. With the advent of high-precision experiments, most data are published in their 'natural' energy axis, and $\mathrm{CRDB}$ enables the following energy axes: $R, E_{k}, E_{k / n}, E_{\mathrm{tot}}$, and $E_{\mathrm{tot}} / A$.

For isotopic fluxes, the mass, atomic number, and charge are uniquely defined, so that conversions between different energy axes are exact and enabled in CRDB - both the energy and the flux are modified. We list in Table A1 the formulae used to move from a native energy axis $E$ into a queried axis $E^{*}$, and how the converted CR flux $d J / d E^{*}$ is linked to the original data $d J / d E$.

Table A1. Conversion between CRDB energy axes. The columns are (i) the native energy axis $(E)$, (ii) the queried axis $E^{*}$ to which to convert, (iii) the relation between $E^{*}$ and $E$, (iv) the relation between the converted flux $d J / d E^{*}$ and the native flux $d J / d E$, and (v) $\beta=v / c$ expressed as a function of the native energy axis $E$. In the formulae below, $m$ is the $C R$ mass in $\mathrm{GeV}$, and energies are in $\mathrm{GeV}$ ( $E_{k}$ and $\left.E_{\text {tot }}\right), \mathrm{GeV} / \mathrm{n}\left(E_{k / n}\right), \mathrm{GV}(R)$, or $\mathrm{GeV} / \mathrm{A}\left(E_{\mathrm{tot} / \mathrm{A}}\right)$.

\begin{tabular}{|c|c|c|c|c|}
\hline Native $E$ & Queried $E^{*}$ & $E^{*}=f(E, A, m, Z)$ & $\frac{\left(d J / d E^{*}\right)}{(d J / d E)}$ & $\beta_{E}$ \\
\hline $\begin{array}{l}E_{k / n} \\
E_{k / n} \\
E_{k / n} \\
E_{k / n} \\
\end{array}$ & $\begin{array}{l}E_{k} \\
R \\
E_{\text {tot }} \\
E_{\text {tot/A }} \\
\end{array}$ & $\begin{array}{l}E_{k}=A E_{k / n} \\
R=\sqrt{A E_{k / n}\left(A E_{k / n}+2 m\right)} /|Z| \\
E_{\text {tot }}=A E_{k / n}+m \\
E_{\text {tot } / \mathrm{A}}=E_{k / n}+m / A \\
\end{array}$ & $\begin{array}{l}1 / A \\
|Z| \beta_{E_{k / n}} / A \\
1 / A \\
1 \\
\end{array}$ & $\beta_{E_{k / n}}=\frac{\sqrt{A E_{k / n}\left(A E_{k / n}+2 m\right)}}{\left(A E_{k / n}+m\right)}$ \\
\hline $\begin{array}{l}E_{k} \\
E_{k} \\
E_{k} \\
E_{k}\end{array}$ & $\begin{array}{l}E_{k / n} \\
R \\
E_{\text {tot }} \\
E_{\text {tot } / \mathrm{A}}\end{array}$ & $\begin{array}{l}E_{k / n}=E_{k} / A \\
R=\sqrt{E_{k}\left(E_{k}+2 m\right)} /|Z| \\
E_{\mathrm{tot}}=E_{k}+m \\
E_{\mathrm{tot} / \mathrm{A}}=\left(E_{k}+m\right) / A\end{array}$ & $\begin{array}{l}\text { A } \\
|Z| \beta_{E} \\
1 \\
A\end{array}$ & $\beta_{E_{k}}=\frac{\sqrt{E_{k}\left(E_{k}+2 m\right)}}{\left(E_{k}+m\right)}$ \\
\hline $\begin{array}{l}R \\
R \\
R \\
R\end{array}$ & $\begin{array}{l}E_{k / n} \\
E_{k} \\
E_{\text {tot }} \\
E_{\text {tot } / \mathrm{A}}\end{array}$ & $\begin{array}{l}E_{k / n}=\sqrt{\left.(R Z)^{2}+m^{2}\right)-m} / A \\
E_{k}=\sqrt{\left(R Z^{2}\right)+m^{2}}-m \\
E_{\mathrm{tot}}=\sqrt{(R Z)^{2}+m^{2}} \\
E_{\mathrm{tot} / \mathrm{A}}=\sqrt{(R Z)^{2}+m^{2}} / A\end{array}$ & $\begin{array}{l}A /\left(|Z| \beta_{R}\right) \\
1 /\left(|Z| \beta_{R}\right) \\
1 /\left(|Z| \beta_{R}\right) \\
A /\left(|Z| \beta_{R}\right)\end{array}$ & $\beta_{R}=\frac{R}{\sqrt{R^{2}+(m / Z)^{2}}}$ \\
\hline $\begin{array}{l}E_{\mathrm{tot}} \\
E_{\mathrm{tot}} \\
E_{\mathrm{tot}} \\
E_{\mathrm{tot}}\end{array}$ & $\begin{array}{l}E_{k / n} \\
E_{k} \\
R \\
E_{\text {tot } / \mathrm{A}}\end{array}$ & $\begin{array}{l}E_{k / n}=\left(E_{\mathrm{tot}}-m\right) / A \\
E_{k}=\left(E_{\mathrm{tot}}-m\right) \\
R=\sqrt{E_{\mathrm{tot}}^{2}-m^{2}} /|Z| \\
E_{\mathrm{tot} / \mathrm{A}}=E_{\mathrm{tot}} / A\end{array}$ & $\begin{array}{l}A \\
1 \\
|Z| \beta_{E_{\text {tot }}} \\
A\end{array}$ & $\beta_{E_{\mathrm{tot}}}=\frac{\sqrt{E_{\mathrm{tot}}^{2}-m^{2}}}{E_{\mathrm{tot}}}$ \\
\hline $\begin{array}{l}E_{\text {tot } / \mathrm{A}} \\
E_{\text {tot } / \mathrm{A}} \\
E_{\text {tot/A }} \\
E_{\text {tot } / \mathrm{A}}\end{array}$ & $\begin{array}{l}E_{k / n} \\
E_{k} \\
R \\
E_{\text {tot }}\end{array}$ & $\begin{array}{l}E_{k / n}=E_{\mathrm{tot} / \mathrm{A}}(m / A) \\
E_{k}=A E_{\mathrm{tot} / \mathrm{A}}-m \\
R=\sqrt{\left(A E_{\mathrm{tot} / \mathrm{A}}\right)^{2}-m^{2}} /|Z| \\
E_{\mathrm{tot}}=A E_{\mathrm{tot} / \mathrm{A}}\end{array}$ & $\begin{array}{l}1 \\
1 / A \\
|Z| \beta_{E_{\mathrm{tot} / \mathrm{A}}} / A \\
1 / A\end{array}$ & $\beta_{E_{\mathrm{tot} / \mathrm{A}}}=\frac{\sqrt{E_{\mathrm{tot} / \mathrm{A}}^{2}-(m / A)^{2}}}{E_{\mathrm{tot} / \mathrm{A}}}$ \\
\hline
\end{tabular}


For measurements that can only resolve fluxes from elements (or groups of elements), the conversion is no longer possible, because the mandatory (energy-dependent) isotopic content to do so is unknown. Nevertheless, assuming that elements (or groups of elements) are dominated by a single isotope, an approximate conversion can be provided. As this proves useful for several users, this approximate conversion is enabled since $C R D B V 1$.2. It takes as the dominant isotope the most abundant one in the Solar system [130], and this is defined, along with the associated values for $A$, $Z$, and $m$, in the ISOTOPE_PROXY table of the database (see Figure 1). We list in Table A2 the proxies enabled for the new CR_QUANTITY names introduced in CRDB V4.0.

Table A2. List of new CRDB names for CR_QUANTITY (see Section 2.1), along with their proxy (i.e., isotope) used to perform approximate energy axis conversions ( $\mathrm{n} / \mathrm{a}$ indicates that no energy conversion at all is enabled).

\begin{tabular}{|c|c|c|}
\hline CRDB Name & Description & Proxy for $E$ Conversion \\
\hline \multicolumn{3}{|c|}{ For UHCR data (Section 3.2) } \\
\hline HS-group & Heavy secondary $(70 \leq Z \leq 73)$ & $\mathrm{n} / \mathrm{a}$ \\
\hline LS-group & Light secondary $(62 \leq Z \leq 69)$ & $\mathrm{n} / \mathrm{a}$ \\
\hline Pt-group & Platinum group of element $(74 \leq Z \leq 80)$ & $\mathrm{n} / \mathrm{a}$ \\
\hline $\mathrm{Pb}$-group & Lead group of elements $(81 \leq Z \leq 87)$ & $\mathrm{n} / \mathrm{a}$ \\
\hline Subactinides & $\mathrm{Pt}+\mathrm{Pb}$ groups $(74 \leq \mathrm{Z} \leq 87)$ & $\mathrm{n} / \mathrm{a}$ \\
\hline Actinides & All the heaviest $C$ Rs $(Z \geq 88)$ & $\mathrm{n} / \mathrm{a}$ \\
\hline Zgeq70 & CRs heavier than charge $70(Z \geq 70)$ & $\mathrm{n} / \mathrm{a}$ \\
\hline \multicolumn{3}{|c|}{ For upper limits on antinuclei (Section 3.3) } \\
\hline Zgeq1 & Flux of all CR nuclei $Z \geq 1$ & ${ }^{1} \mathrm{H}$ \\
\hline Zgeq1-bar & Flux of all $C R$ antinuclei $Z \leq-1$ & $\overline{{ }^{1} \mathrm{H}}$ \\
\hline Zgeq2 & Flux of all $C R$ nuclei $Z \geq 2$ & ${ }^{4} \mathrm{He}$ \\
\hline Zgeq2-bar & Flux of all $C R$ antinuclei $Z \leq-2$ & $\overline{{ }^{4} \mathrm{He}}$ \\
\hline $\mathrm{H}-\mathrm{BAR}$ & Antihydrogen flux $(\overline{\mathrm{H}})$ & $\overline{{ }^{1} \mathrm{H}}$ \\
\hline $1 \mathrm{H}-\mathrm{BAR}$ & Antiproton flux $(\bar{p})$ & $\mathrm{n} / \mathrm{a}$ \\
\hline $2 \mathrm{H}-\mathrm{BAR}$ & Antideuteron flux $\left(\overline{{ }^{2} \mathrm{H}}\right)$ & $\mathrm{n} / \mathrm{a}$ \\
\hline $\mathrm{He}-\mathrm{BAR}$ & Antihelium flux $(\overline{\mathrm{He}})$ & $\overline{4} \mathrm{He}$ \\
\hline$\cdots$ & (aown to tsotopes ana elements $Z=-8$ ) & \\
\hline \multicolumn{3}{|c|}{ For UHECR data (Section 3.4) } \\
\hline $\mathrm{H}-\mathrm{He}$-group & Flux inferred from mixture of $\mathrm{H}$ and $\mathrm{He}$ in air-shower simulations & $\mathrm{n} / \mathrm{a}$ \\
\hline $\mathrm{C}-\mathrm{Fe}$-group & Flux inferred from mixture of $\mathrm{C}$ to $\mathrm{Fe}$ in air-shower simulations & $\mathrm{n} / \mathrm{a}$ \\
\hline $\mathrm{N}$-group & Flux inferred from $\mathrm{N}$ in air-shower simulations & ${ }^{14} \mathrm{~N}$ \\
\hline O-group & Flux inferred from $\mathrm{O}$ in air-shower simulations & ${ }^{16} \mathrm{O}$ \\
\hline Al-group & Flux inferred from $\mathrm{Al}$ in air-shower simulations & ${ }^{27} \mathrm{Al}$ \\
\hline Si-group & Flux inferred from $\mathrm{Si}$ in air-shower simulations & ${ }^{28} \mathrm{Si}$ \\
\hline Fe-group & Flux inferred from $\mathrm{Fe}$ in air-shower simulations & ${ }^{56} \mathrm{Fe}$ \\
\hline $\mathrm{O}-\mathrm{Fe}$-group & Flux inferred from a mixture of $\mathrm{O}$ to $\mathrm{Fe}$ in air-shower simulations & $\mathrm{n} / \mathrm{a}$ \\
\hline AllParticles & Flux of all particles (sum over all isotopes and species) & $\mathrm{n} / \mathrm{a}$ \\
\hline
\end{tabular}

\section{Appendix A.5. Data Taking Period (Possible) Overlap}

By default, for a given sub-exp, the data extraction tool returns all requested data, regardless of their data taking periods. We caution the user to check for any overlap between dates. Because of the time-dependent Solar activity, datasets from different though overlapping data taking periods can be of interest in the context of Solar modulation studies. However, if one is only interested in the accumulated statistics, one should only consider datasets accumulated on the longest time period.

In any case, datasets from overlapping data taking periods are not independent. The user has to critically decide which data sets are relevant for her/his analysis, and refine the selection criteria accordingly. 


\section{Appendix B. List of Sub-Experiments and Publications}

In this appendix, we provide several lists of experiments and their associated publications, for several types of CR entries: UHECRs (Appendix B.1), antinuclei (Appendix B.2), antiprotons (Appendix B.3), leptons (Appendix B.4), nuclei $Z \leq 30$ (Appendix B.5), and UHCRs (Appendix B.6). We sort them by experiment type (balloon, space, or ground) and in alphabetical order.

\section{Appendix B.1. UHECR Data}

\section{Ground}

- Pierre Auger Observatory Hybrid (2004/01-2014/12): Aab et al. 2015 [121]

- Pierre Auger Observatory FDSIBYLL-2.1 (2004/12-2012/12): Aab et al. 2014 [109]

- Pierre Auger Observatory FDQGSJetII.04 (2004/12-2012/12): Aab et al. 2014 [109]

- Pierre Auger Observatory FDEPOS-LHC (2004/12-2012/12): Aab et al. 2014 [109]

- Telescope Array Hybrid (2008/01-2015/05): Ivanov 2015 [122]

- KASCADE-Grande QGSJetII.04 (2003/01-2009/03): Schoo et al. 2015 [120]

- TUNKA-133Array QGSJet01 (2009/10-2012/04): Prosin et al. 2014 [110]

- IceCube Hybrid SIBYLL-2.1 (2010/06-2013/05): Rawlins and IceCube Collaboration 2015 [108]

- IceCubeIceTop SIBYLL-2.1 (2010/06-2013/05): Rawlins and IceCube Collaboration 2015 [108]

- H.E.S.S. Average of QGSJet01 and SIBILL-2.1 (2004/01-2006/12): Aharonian et al. 2007 [131]

Appendix B.2. Upper Limits on Antinuclei $(Z<-1$ and $A \geq 2)$

Balloon

- $\quad$ Balloon (1957/09): Aizu et al. 1961 [96]

- $\quad$ Balloon (1969/08): Golden et al. 1971, 1974 [98,102]

- Balloon (1970/05): Verma et al. 1972 [101]

- Balloon (1970/07): Evenson 1972 [100]

- Balloon (1970/XX): Greenhill et al. 1971 [97]

- Balloon (1970/09+1971/05): Buffington et al. 1972 [99]

- Balloon (1970/09+1971/05+1972/09): Smoot et al. 1975 [86]

- Balloon (1975/09+1976/05): Badhwar et al. 1978 [87]

- Balloon (1980/06): Buffington et al. 1981 [77]

- $\quad$ Balloon (1987/08): Golden et al. 1997 [89]

- $\quad$ BESS95 (1995/07): Ormes et al. 1997 [88]

- $\operatorname{BESS97~(1997/07):~Fuke~et~al.~} 2005$ [95]

- $\operatorname{BESS98}$ (1998/07): Fuke et al. 2005 [95]

- $\operatorname{BESS99~(1999/08):~Fuke~et~al.~} 2005$ [95]

- $\operatorname{BESS00~(2000/08):~Fuke~et~al.~} 2005$ [95]

- $\quad$ BESS-TeV (2002/08): Sasaki et al. 2008 [93]

- $\quad$ BESS-PolarI (2004/12): Sasaki et al. 2008 [93]

- BESS-PolarII (2007/12-2008/01): Abe et al. 2012 [10]

- BESS-COMBINED (1993-1995): Saeki et al. 1998 [90]

- BESS-COMBINED (1993-2000): Sasaki et al. 2002 [92]

- BESS-COMBINED (1993-2004): Sasaki et al. 2008 [93]

- BESS-COMBINED (1993-2007): Abe et al. 2012 [10]

- BESS-COMBINED (1997-2000): Fuke et al. 2005 [95]

Space

- AMS01 (1998/06): Alcaraz et al. 1999 [91], Cristinziani 2002 [103]

- PAMELA (2006/07-2009/12): Mayorov et al. 2011 [94] 
Appendix B.3. Antiproton Data

\section{Balloon}

- Balloon (1979/06): Golden et al. 1979 [132]

- Balloon (1979/06): Golden et al. 1984 [133]

- Balloon (1980/06): Buffington et al. 1981 [77]

- Balloon (1984/06+1984/07): Bogomolov et al. 1987 [134]

- $\operatorname{BESS93~(1993/07):~Moiseev~et~al.~} 1997$ [135]

- $\operatorname{BESS95}$ (1995/07): Matsunaga et al. 1998 [136]

- $\quad \operatorname{BESS97~(1997/07):~Orito~et~al.~} 2000$ [137]

- $\quad$ BESS98 (1998/07): Maeno et al. 2001 [138]

- $\operatorname{BESS99~(1999/08):~Asaoka~et~al.~} 2002$ [139]

- $\operatorname{BESS00~(2000/08):~Asaoka~et~al.~} 2002$ [139]

- $\quad$ BESS-TeV (2002/08): Haino et al. 2005 [140]

- $\quad$ BESS-PolarI (2004/12): Abe et al. 2008 [141]

- $\quad$ BESS-PolarII (2007/12-2008/01): Abe et al. 2012 [142]

- CAPRICE94 (1994/08): Boezio et al. 1997 [143]

- CAPRICE98 (1998/05): Boezio et al. 2001 [144]

- HEAT-pbar (2000/06): Beach et al. 2001 [145]

- IMAX92 (1992/07): Mitchell et al. 1996 [146]

- MASS91 (1991/09): Basini 1999 [147]

- MASS91 (1991/09): Hof et al. 1996 [148]

Space

- AMS01 (1998/06): Aguilar et al. 2002 [149]

- $\quad \operatorname{AMS02~(2011/05-2015/05):~Aguilar~et~al.~} 2016$ [79]

- PAMELA (2006/07-2008/06): Adriani et al. 2009 [150]

- PAMELA (2006/07-2008/12): Adriani et al. 2010 [151]

- PAMELA (2006/07-2009/12): Adriani et al. 2013 [152]

Appendix B.4. Lepton Data

Balloon

- Balloon (1963/04): Daniel and Stephens 1965 [153]

- Balloon (1963/07): Freir and Waddington 1965 [154]

- Balloon (1964/07): L'Heureux 1967 [155]

- Balloon (1965/06+1965/07): L’Heureux 1967 [155]

- Balloon (1965/07): Webber et al. 1973 [156]

- Balloon (1965/07): Bleeker et al. 1965 [157]

- Balloon (1965/07+1965/08+1966/06: Fanselow 1968 [158]

- Balloon (1965/07+1965/08+1966/06: Fanselow et al. 1969 [159]

- Balloon (1965/07+1966/07): Beedle and Webber 1968 [160]

- Balloon (1966/03): Anand et al. 1968 [161]

- Balloon (1966/06): L'Heureux and Meyer 1968 [162]

- Balloon (1966/07): Webber et al. 1973 [156]

- Balloon (1966/07): Earl et al. 1972 [163]

- Balloon (1966/08): Bleeker et al. 1970 [164]

- Balloon (1966/09): Earl et al. 1972 [163]

- Balloon (1966/09): Danjo et al. 1968 [165]

- Balloon (1967/06+1967/07): Israel and Vogt 1968 [166] 
- Balloon (1967/07): Agrinier et al. 1969 [167]

- Balloon (1967/07): Earl et al. 1972 [163]

- Balloon (1967/08): Bleeker et al. 1970 [164]

- Balloon (1967/11+1968/06): Fanselow et al. 1971 [168]

- Balloon (1968+...+2001): Kobayashi et al. 2012 [169]

- Balloon (1968/05): Anand et al. 1973 [170]

- Balloon (1968/06): Scheepmaker and Tanaka 1971 [171]

- Balloon (1968/06+1968/07): Fulks 1975 [172]

- Balloon (1968/07): Beuermann et al. 1969 [173]

- Balloon (1968/07): Beuermann et al. 1969 [173]

- Balloon (1968/07): Webber et al. 1973 [156]

- Balloon (1968/07): Bleeker et al. 1970 [164]

- Balloon (1969/04+1970/11): Silverberg et al. 1973 [174]

- Balloon (1969/06+1969/07): Fulks 1975 [172]

- Balloon (1969/07): Webber et al. 1973 [156]

- Balloon (1969/09+1973/05): Meegan and Earl 1975 [175]

- Balloon (1970/05+1970/09): Muller and Meyer 1973 [176]

- Balloon (1970/06+1970/07): Fulks 1975 [172]

- Balloon (1970/11): Silverberg 1976 [177]

- Balloon (1971/06+1971/07): Fulks 1975 [172]

- Balloon (1971/07): Webber et al. 1973 [156]

- Balloon (1971/07+1972/07): Webber et al. 1973 [156]

- Balloon (1972/07): Daugherty et al. 1975 [178]

- Balloon (1972/07): Webber et al. 1973 [156]

- Balloon (1972/07): Fulks 1975 [172]

- Balloon (1972/10): Freier et al. 1977 [179]

- Balloon (1972/10): Silverberg 1976 [177]

- Balloon (1972/11+1973/05): Buffington et al. 1975 [180]

- Balloon (1973/06): Ishii et al. 1973 [181]

- Balloon (1973/07): Caldwell et al. 1975 [182]

- Balloon (1974/07): Caldwell et al. 1977 [183]

- Balloon (1974/07+1974/08): Hartman and Pellerin 1976 [184]

- Balloon (1975/07): Caldwell et al. 1977 [183]

- Balloon (1975/10): Prince 1979 [185]

- Balloon (1976/05): Golden et al. 1984 [186]

- Balloon (1976/05): Golden et al. 1987 [187]

- Balloon (1977/07): Evenson et al. 1979 [188]

- Balloon (1979/08): Evenson and Meyer 1984 [189]

- Balloon (1980/10): Tang 1984 [190]

- Balloon (1982/08): Evenson and Meyer 1984 [189]

- Balloon (1982/10): Garcia-Munoz et al. 1986 [191]

- Balloon (1984/04): Mueller and Tang 1987 [192]

- Balloon (1984/09): Garcia-Munoz et al. 1986 [191]

- Balloon (1987/08): Evenson et al. 1995 [193]

- Balloon (1990/08): Evenson et al. 1995 [193]

- Balloon (1992/08): Evenson et al. 1995 [193]

- Balloon (1994/08): Evenson et al. 1995 [193] 
- $\quad$ AESOP94 (1994/08): Clem et al. 1996 [194]

- AESOP97+98 (1997/09+1998/08): Clem et al. 2000 [195]

- $\operatorname{AESOP99~(1999/08):~Clem~and~Evenson~} 2002$ [196]

- AESOP00 (2000/08): Clem and Evenson 2002 [196]

- $\quad \operatorname{AESOP02~(2002/08):~Clem~and~Evenson~} 2004$ [197]

- $\operatorname{AESOP06~(2006/08):~Clem~and~Evenson~} 2009$ [198]

- ATIC01\&02 (2001/01+2003/01): Chang et al. 2008 [199]

- $\quad$ BETS04 (2004/01): Yoshida et al. 2008 [200]

- BETS97\&98 (1997/06+1998/05): Torii et al. 2001 [201]

- CAPRICE94 (1994/08): Boezio et al. 2000 [202]

- CAPRICE98 (1998/05): Boezio et al. 2001 [203]

- HEAT-pbar (2000/06): Beatty et al. 2004 [204]

- $\quad$ HEAT94 (1994/05): Barwick et al. 1998 [205]

- HEAT94 (1994/05): Barwick et al. 1997 [206]

- HEAT94\&95 (1994/05+1995/08): DuVernois et al. 2001 [207]

- HEAT94\&95 (1994/05+1995/08): Barwick et al. 1997 [206]

- $\operatorname{HEAT95~(1995/08):~DuVernois~et~al.~} 2001$ [207]

- $\operatorname{HEAT95~(1995/08):~Barwick~et~al.~} 1997$ [206]

- $\operatorname{MASS89}$ (1989/09): Golden et al. 1994 [208]

- MASS91 (1991/09): Grimani et al. 2002 [209]

- $\operatorname{TS93~(1993/09):~Golden~et~al.~} 1996$ [210]

Space

- AMS01-BremsstrahlungPhotons (1998/06): Aguilar et al. 2007 [211]

- AMS01-singleTrack (1998/06): Alcaraz et al. 2000 [212]

- $\operatorname{AMS02~(2011/05-2012/12):~Aguilar~et~al.~} 2013$ [213]

- $\operatorname{AMS02~(2011/05-2013/11):~Aguilar~et~al.~} 2014$ [214]

- $\quad \operatorname{AMS02~(2011/05-2015/05):~Aguilar~et~al.~2016~[79]~}$

- $\operatorname{AMS02~(2011/05-2017/11):~Aguilar~et~al.~} 2019$ [215]

- $\operatorname{AMS02~(2011/05-2018/05):~Aguilar~et~al.~} 2020$ [125]

- AMS02 time series: Aguilar et al. 2018 [48]

- CALET (2015/10-2017/06): Adriani et al. 2017 [216]

- CALET (2015/10-2017/11): Adriani et al. 2018 [217]

- DAMPE (2015/12-2017/06): Ambrosi et al. 2017 [218]

- Fermi-LAT-LE (2008/06-2009/06): Ackermann et al. 2010 [219]

- Fermi-LAT-HE (2008/06-2009/06): Ackermann et al. 2010 [219]

- Fermi-LAT-LE (2008/08-2015/06): Abdollahi et al. 2017 [220]

- Fermi-LAT-HE (2008/08-2015/06): Abdollahi et al. 2017 [220]

- $\quad \operatorname{IMP1}$ (1963/11-1964/05): Cline et al. 1964 [221]

- $\quad \operatorname{IMP3}(1965 / 07-1966 / 03)$ : Fan et al. 1968 [222]

- ISEE3-MEH (1978/08-1979/02): Evenson et al. 1979 [188]

- ISEE3-MEH (1979/02-1980/03): Evenson et al. 1981 [223]

- $\quad$ ISEE3-MEH (1980/03-1981/03): Evenson et al. 1981 [223]

- OGO5 (1968/04-1968/05): Burger and Swanenburg 1974 [224]

- $\operatorname{OGO5~(1968/04-1969/04):~L'Heureux~et~al.~} 1972$ [41]

- $\operatorname{OGO5~(1968/06-1968/10):~Fulks~} 1975$ [172]

- $\operatorname{OGO5~(1969/06-1969/07):~Fulks~} 1975$ [172]

- $\operatorname{OGO5~(1969/06-1969/07):~Burger~and~Swanenburg~} 1973$ [225] 
- $\operatorname{OGO5~(1970/06-1970/07):~Fulks~} 1975$ [172]

- OGO5 (1970/06-1970/07): Burger and Swanenburg 1973 [225]

- $\operatorname{OGO5~(1971/05-1971/08):~Fulks~} 1975$ [172]

- $\operatorname{OGO5~(1971/07-1971/08):~Burger~and~Swanenburg~} 1973$ [225]

- $\operatorname{OGO5~(1972/06):~Fulks~} 1975$ [172]

- OGO5 (1972/06-1972/07): Burger and Swanenburg 1974 [224]

- PAMELA (2006/07-2006/11): Adriani et al. 2015 [226]

- PAMELA (2006/07-2008/02): Adriani et al. 2009 [227]

- PAMELA (2006/07-2008/12): Adriani et al. 2010 [228]

- PAMELA (2006/07-2009/12): Adriani et al. 2013 [229]

- PAMELA (2006/07-2010/01): Adriani et al. 2011 [230]

- PAMELA time series: Adriani et al. 2015 [226]

- Pioneer8\&9 (1967/12-1969/04): Webber et al. 1973 [231]

- Ulysses-KET (1990/10-1991/02): Rastoin et al. 1996 [232]

- Ulysses-KET (1992/07-1992/10): Rastoin et al. 1996 [232]

- Ulysses-KET (1993/07-1993/10): Rastoin et al. 1996 [232]

- Ulysses-KET (1994/07-1994/10): Rastoin et al. 1996 [232]

- Voyager1-HET (2010/01-2010/03): Caballero-Lopez et al. 2010 [233]

- Voyager2-HET (2010/01-2010/03): Caballero-Lopez et al. 2010 [233]

- Voyager1-BSe (2012/09-2012/12): Stone et al. 2019 [234]

- Voyager1-HET-Bse (2012/12-2015/06): Cummings et al. 2016 [235]

- Voyager1-TET (2012/09-2012/12): Stone et al. 2019 [234]

- Voyager1-TET (2012/12-2015/06): Cummings et al. 2016 [235]

- Voyager2-BSe (2019/03-2019/07): Stone et al. 2019 [234]

- Voyager2-TET (2019/03-2019/07): Stone et al. 2019 [234]

Ground

- H.E.S.S. (2004/10-2007/08): Aharonian et al. 2008 [236]

- H.E.S.S.-LEAnalysis (2004/10-2005/12): Aharonian et al. 2009 [237]

Appendix B.5. Data for Nuclei $(Z \leq 30)$

These data are the most numerous in terms of experiments and publications. For readability, we further group them by their experiment name below.

$\underline{\text { Balloon }}$

- Balloon $(1950,1954,1957)$

- Balloon (1950/10): Fowler et al. 1957 [238]

- Balloon (1954/06): Freier et al. 1958 [239]

- Balloon (1957/05): Fowler et al. 1957 [238]

- Balloon (1957/06): Aizu et al. 1961 [96]

- Balloon (1957/09): Aizu et al. 1961 [96]

- $\quad$ Balloon $(1955,1956,1958,1959)$

- Balloon (1955/07): McDonald 1956 [240]

- Balloon (1956/03): McDonald and Webber 1959 [241]

- Balloon (1956/08): McDonald 1957 [242]

- Balloon (1956/09): Webber and McDonald 1964 [243]

- Balloon (1958/02): McDonald 1959 [244]

- Balloon (1958/07): McDonald and Webber 1960 [245] 
- Balloon (1959/05): Webber and McDonald 1964 [243]

- Balloon (1959/06): Webber and McDonald 1964 [243]

- $\quad$ Balloon $(1957,1958)$

- Balloon (1957/07): Engler et al. 1961 [246]

- Balloon (1958/08): Engler et al. 1961 [247]

- $\quad$ Balloon $(1960,1961)$

- Balloon (1960/09): Meyer and Vogt 1963 [248]

- Balloon (1961/08): Meyer and Vogt 1963 [248]

- Balloon $(1960,1963,1964,1965,1968)$

- Balloon (1960/08): Freier and Waddington 1965 [249]

- Balloon (1964/07): Freier and Waddington 1968 [250]

- $\quad$ Balloon $(1961,1962,1963,1964)$

- Balloon (1961/07): Fichtel et al. 1964 [251]

- Balloon (1962/07): Fichtel et al. 1964 [252]

- Balloon (1963/06): O'dell et al. 1965 [253]

- Balloon (1964/06): Durgaprasad et al. 1967 [254]

- Balloon (1962/05): Foster and Schrautemeier 1967 [255]

- Balloon (1963/04): Anand et al. 1968 [256]

- Balloon $(1963,1964,1967)$

- Balloon (1963/06): Bhatia et al. 1977 [257]

- Balloon (1964/06): Bhatia et al. 1977 [257]

- Balloon (1967/07): Bhatia et al. 1977 [257]

- $\quad$ Balloon $(1963,1965)$

- Balloon (1963/06): Balasubrahmanyan and McDonald 1964 [258]

- Balloon (1965/06): Balasubrahmanyan et al. 1966 [259]

- Balloon $(1963,1965,1966,1967)$

- Balloon (1963/01): Ormes and Webber 1964 [260]

- Balloon (1963/04): Ormes and Webber 1964 [260]

- Balloon (1965/06): Ormes and Webber 1968 [261]

- Balloon (1963/11): Ormes and Webber 1964 [260]

- Balloon (1966/07): Ormes and Webber 1968 [261]

- Balloon (1967/06): Ormes and Webber 1968 [261]

- Balloon (64/03-65/7): Webber and Ormes 1967 [262]

- Balloon (66/07+67/05-06-07): von Rosenvinge et al. 1969 [263]

- Balloon (1964/09): Courtier and Lenney 1966 [264]

- Balloon (1965/05): Hofmann and Winckler 1966, 1967 [265,266]

- Balloon (1965/07+1965/08+1966/06): Hartman 1967 [267]

- Balloon $(1965,1966,1967,1968,1969,1970,1971,1972)$

- Balloon (1965/08): Rygg and Earl 1971 [268]

- Balloon (1966/08): Rygg and Earl 1971 [268]

- Balloon (1967/07): Rygg and Earl 1971 [268]

- Balloon (1968/07): Rygg and Earl 1971 [268]

- Balloon (1969/07): Rygg and Earl 1971 [268]

- Balloon (1970/07): Rygg et al. 1974 [269] 
- Balloon (1971/07): Rygg et al. 1974 [269]

- Balloon (1972/07): Rygg et al. 1974 [269]

- $\quad$ Balloon $(1965,1966,1968,1969,1970)$

- Balloon (1965/06+1966/06): Garrard et al. 1973 [270]

- Balloon (1969/06): Garrard et al. 1973 [270]

- Balloon (1970/06): Garrard et al. 1973 [270]

- $\quad$ Balloon $(1965,1966,1968,1969,1971+1972)$

- Balloon (1965/07): Webber and Chotkowski 1967 [271]

- Balloon (1971/07+1972/07): Webber et al. 1973 [156]

- Balloon (1966/07+1966/08): Badhwar et al. 1967 [272]

- Balloon (1970/07+1970/06): Behrnetz et al. 1976 [273]

- Balloon (1968)

- Balloon (1968/05): Scheepmaker and Tanaka 1971 [171]

- Balloon (1968/07): Singh and Bhatia 1979 [274]

- Ballon (1968...1994)

- Balloon (1973/07): Caldwell et al. 1975 [182]

- Balloon (1974/07): Caldwell et al. 1977 [183]

- Balloon (1975/07): Caldwell et al. 1977 [183]

- Balloon (1977/07): Evenson et al. 1979 [188]

- Balloon (1979/08): Evenson and Meyer 1984 [189]

- Balloon (1982/08): Evenson and Meyer 1984 [189]

- Balloon (1982/10): Garcia-Munoz et al. 1986 [191]

- Balloon (1984/09): Garcia-Munoz et al. 1986 [191]

- Balloon (1987/08): Evenson et al. 1995 [193]

- Balloon (1990/08): Evenson et al. 1995 [193]

- Balloon (1992/08): Evenson et al. 1995 [193]

- Balloon (1994/08): Evenson et al. 1995 [193]

- Balloon (1970)

- Balloon (1970/05): Verma et al. 1972 [101]

- Balloon (1970/06): Bjarle et al. 1979 [275]

- Balloon (1970/07): Webber et al. 1972 [276]

- Balloon (1970/11): Ryan et al. 1972 [277]

- Balloon (1970/09+1971/05): Smith et al. 1973 [278]

- Balloon (1971/05): Apparao 1973 [279]

- Balloon (1971/09+1972/10): Juliusson 1974 [280]

- Balloon (1973/08): Fisher et al. 1976 [281], Hagen et al. 1977 [282], Maehl et al. 1977 [283], Leech and Ogallagher 1978 [284]

- $\quad$ Balloon $(1973+1974+1975)$

- Balloon (1973/09+1974/05): Dwyer 1978 [285]

- Balloon (1973/09+1974/05+...): Dwyer and Meyer 1987 [286]

- Balloon (1975/09+1975/10): Minagawa 1981 [287]

- Balloon (1974/10): Scarlett et al. 1978 [288]

- Balloon (1974/07+/08+1976/09): Lezniak and Webber 1978 [289]

- Balloon (1975/12): Bogomolov et al. 1979 [76]

- $\quad$ Balloon $(1976,1979)$ 
- $\quad$ Balloon (1976/09): Derrickson et al. 1992 [290]

- Balloon (1976/10): Simon et al. 1980 [291]

- Balloon (1979/06): Webber et al. 1987 [292]

- Balloon (1977)

- Balloon (1977/05): Buffington et al. 1978 [293]

- Balloon (1977/07): Webber and Yushak 1983 [294], Webber et al. 1987 [292]

- Balloon (1977/09): Webber and Kish 1979 [295], Webber 1981, 1982 [296,297], Webber and Yushak 1979 [298]

- $\quad$ Balloon (1979/06): Golden et al. 1979, 1984 [132,133]

- Balloon (1980/06): Buffington et al. 1981 [77]

- Balloon (1981)

- $\quad$ Balloon (1981/04): Jordan 1985 [299]

- Balloon (1981/09): Webber et al. 1985 [300,301]

- Balloon (1984/06+1984/07): Bogomolov et al. 1987 [134]

- Balloon (1987/05+1988/05+1989/05+1991/05): Ichimura et al. 1993 [302]

- Balloon (1989/09): Hatano et al. 1995 [303]

- Balloon (1989+1991)

- Balloon-EWAsym (1989/05+1991/05): Kamioka et al. 1997 [304]

- Balloon-OpeningAngle (1989/05+1991/05): Kamioka et al. 1997 [304]

- Balloon (1990/07): Bogomolov et al. 1995 [305]

- Balloon (1991/09): Buckley et al. 1994 [306]

- ALICE

- ALICE (1987/08): Hesse et al. 1996 [307]

- ALICE (1987/08+1987/08): Esposito et al. 1992 [308]

- $\quad \operatorname{ATIC02~(2003/01):~Panov~et~al.~2008,~} 2009$ [114,309]

- $\quad$ BESS

- $\quad$ BESS93 (1993/07): Moiseev et al. 1997 [135], Wang et al. 2002 [310]

- $\quad$ BESS94 (1994/07): Seo et al. 2001 [311], Myers et al. 2003, 2005 [312,313], Kim et al. 2013 [314]

- BESS95 (1995/07): Matsunaga et al. 1998 [136], Seo et al. 2001 [311], Myers et al. 2003, 2005 [312,313], Kim et al. 2013 [314]

- $\quad$ BESS97 (1997/07): Orito et al. 2000 [137], Myers et al. 2003, 2005 [312,313], Shikaze et al. 2007 [315], Kim et al. 2013 [314]

- $\quad$ BESS98 (1998/07): Sanuki et al. 2000 [316], Maeno et al. 2001 [138], Myers et al. 2003, 2005 [312,313], Shikaze et al. 2007 [315]

- $\quad$ BESS99 (1999/08): Asaoka et al. 2002 [139], Shikaze et al. 2007 [315]

- $\quad$ BESS00 (2000/08): Asaoka et al. 2002 [139], Shikaze et al. 2007 [315], Kim et al. 2013 [314]

- $\quad$ BESS-TeV (2002/08): Haino et al. 2004 [317], Haino et al. 2005 [140], Shikaze et al. 2007 [315]

- BESS-PolarI (2004/12): Abe et al. 2008, 2015 [141,318]

- BESS-PolarII (2007/12-2008/01): Abe et al. 2012, 2015 [142,318]

- $\quad$ CAPRICE

- CAPRICE94 (1994/08): Boezio et al. 1997, 1999 [143,319]

- CAPRICE98 (1998/05): Boezio et al. 2001, 2003 [144,320], Mocchiutti et al. 2003 [321], Papini et al. 2004 [322]

- CREAM

- CREAM-I (2004/12-2005/01): Ahn et al. 2008 [323], Yoon et al. 2011 [116] 
- CREAM-I+III (2004+2007): Yoon et al. 2017 [324]

- CREAM-II (2005/12-2006/01): Ahn et al. 2009, 2010 [325,326]

- CREAM-III (2007/12-2008/01): Yoon et al. 2017 [324]

- CRISIS (1977/05): Freier et al. 1980 [327], Young et al. 1981 [328]

- $\quad$ HEAT-pbar (2000/06): Beach et al. 2001 [145]

- $\quad$ HEIST (1988/08): Gibner et al. 1992 [329]

- IMAX92 (1992/07): Mitchell et al. 1996 [146], Reimer et al. 1998 [330], Menn et al. 2000 [331], de Nolfo et al. 2000 [332]

- $\quad$ IRIS (1976/09): Tarle et al. 1979 [333,334]

- $\operatorname{ISOMAX~(1998/08):~Hams~et~al.~} 2004$ [335]

- JACEE (1979+...+1995): Asakimori et al. 1998 [336]

- $\operatorname{LEAP}(1987 / 08)$ : Seo et al. 1991 [337]

- MASS

- $\operatorname{MASS89~(1989/09):~Webber~et~al.~} 1991$ [338]

- $\quad$ MASS91 (1991/09): Hof et al. 1996 [148], Bellotti et al. 1999 [339], Basini 1999 [147]

- $\quad \operatorname{MUBEE}(1975 / 09+1978 / 08+1986 / 07+1987 / 07)$ : Zatsepin et al. 1993 [340]

- $\quad$ RICH-II (1997/10): Diehl et al. 2003 [341]

- RUNJOB (1995+1996+1997+1998+1999): Derbina et al. 2005 [118]

- $\quad$ SMILI

- $\quad$ SMILI-I (1989/09): Beatty et al. 1993 [44]

- $\quad$ SMILI-II (1991/07): Ahlen et al. 2000 [342], Wefel et al. 1995 [343]

- tRACER

- TRACER99: Gahbauer et al. 2004 [344]

- TRACER03 (2003/12): Ave et al. 2008 [345]

- TRACER06 (2006/07): Obermeier et al. 2011 [346]

Space

- $\mathrm{ACE}$

- ACE-CRIS (1997/08-1998/04): George et al. 2009 [347], Lave et al. 2013 [348]

- ACE-CRIS (1997/08-1998/12): Wiedenbeck et al. 1999 [349]

- $\quad$ ACE-CRIS (1997/08-1999/07): Yanasak et al. 2001 [350], Ogliore et al. 2009 [351]

- ACE-CRIS (1997/12-1999/09): Binns et al. 2005 [352]

- ACE-CRIS (1997/12-2000/07): Binns et al. 2001 [353]

- ACE-CRIS (1998/01-1999/01): de Nolfo et al. 2006 [354]

- $\quad$ ACE-CRIS (2001/05-2003/09): George et al. 2009 [347], Lave et al. 2013 [348]

- ACE-CRIS (2009/03-2010/01): Lave et al. 2013 [348]

- $\quad$ ACE-SIS (1997/08-1999/07): Yanasak et al. 2001 [350]

- $\quad$ AMS01

- $\operatorname{AMS01~(1998/06):~Alcaraz~et~al.~} 2000$ [355,356], Xiong et al. 2003 [357], Aguilar et al. 2002, 2010, $2011[149,358,359]$

- $\quad$ AMS02

- $\quad$ AMS02 (2011/05-2011/06): Aguilar et al. $2018[47,48]$

- $\quad$ AMS02 (2011/05-2011/08): Aguilar et al. 2019 [111]

- $\quad$ AMS02 (2011/05-2013/11): Battiston 2014 [360], Aguilar et al. 2014, 2015a [13,14,214]

- $\quad$ AMS02 (2011/05-2016/05): Aguilar et al. 2016, 2017, 2018 [15,123,124,361]

- $\quad$ AMS02 (2011/05-2017/05): Aguilar et al. 2018 [48] 
- $\quad$ AMS02 (2011/05-2017/11): Aguilar et al. 2019 [111,362]

- AMS02 time series: Aguilar et al. 2018, $2019[47,111]$

- $\operatorname{CALET}(2015 / 10-2018 / 08)$ : Adriani et al. 2019 [115]

- CRN-Spacelab2 (1985/07-1985/08): Swordy et al. 1990 [363], Mueller et al. 1991 [364]

- cRRES

- CRRES (1990/07-1992/10): Duvernois et al. 1996 [365]

- CRRES (1990/12-1991/03): Clayton et al. 2000 [366]

- Discoverer36 (1961/12): Stone 1964 [367]

- $\quad$ E6 (1974/12-1977/12): Marquardt and Heber 2019 [368]

- EPHIN time series (1995-2014): Kühl et al. 2016 [369]

- Explorer12 (1961/08): Bryant et al. 1962 [370]

- Gemini11 (1966/08): Durgaprasad et al. 1970 [371]

- HEAO3-C2 (1979/10-1980/06): Ferrando et al. 1988 [372], Engelmann et al. 1990 [373]

- IMP

- $\quad$ IMP1 (1963/11-1964/02): Gloeckler 1965 [374]

- $\quad$ IMP1 (1963/11-1964/05): Fan et al. 1965 [375], McDonald and Ludwig 1964 [376]

- $\quad$ IMP1 (1964/02-1964/05): Gloeckler 1965 [374]

- IMP3 (1965/05-1965/06): Balasubrahmanyan et al. 1966 [259]

- $\quad$ IMP3 (1965/05-1965/09): Fan et al. 1966 [377,378], Hsieh 1970 [379]

- $\quad$ IMP3 (1965/06-1965/12): Hsieh et al. 1971 [380]

- $\quad \operatorname{IMP4}(1967 / 06-1967 / 10):$ Hsieh et al. 1971 [380]

- $\quad$ IMP4 (1967/07-1967/10): Hsieh 1970 [379]

- $\quad$ IMP5 (1969/06-1969/09): Hsieh et al. 1971 [380], Mason 1972 [381]

- $\quad$ IMP5 (1969/06-1970/06): Mason 1972 [381]

- $\quad$ IMP5 (1970/04-1970/10): Garcia-Munoz et al. 1975 [382]

- $\quad$ IMP5 (1971/06-1971/09): Garcia-Munoz et al. 1975 [382]

- $\quad$ IMP5 (1972/06-1972/09): Garcia-Munoz et al. 1975 [382]

- $\quad$ IMP7 (1972/09-1972/12): Teegarden et al. 1975 [383]

- $\quad$ IMP7 (1972/10-1976/10): Garcia-Munoz et al. 1979 [384]

- $\quad$ IMP7 (1973/01-1974-10): Mewaldt et al. 1976 [385]

- $\quad \operatorname{IMP7}$ (1973/05-1973/07): Garcia-Munoz et al. 1975 [382]

- $\quad$ IMP7 (1973/05-1973/08): Garcia-Munoz et al. 1975 [382]

- $\quad$ IMP7 (1973/05-1973/12): Garcia-Munoz et al. 1975 [382]

- $\quad$ IMP7\&8 (1972/09-1975/09): Garcia-Munoz et al. 1977 [386,387]

- $\quad$ IMP7\&8 (1973/02-1977/09): Guzik 1981 [388]

- $\quad$ IMP7\&8 (1974/01-1980/05): Garcia-Munoz et al. 1981 [389]

- $\quad$ IMP8 (1974/01-1977/11): Beatty et al. 1985 [390]

- $\quad$ IMP8 (1974/01-1978/10): Garcia-Munoz et al. 1987 [391]

- $\quad$ IMP8 (1974/07-1974/09): Garcia-Munoz et al. 1975 [382]

- $\quad$ IMP8 (1975/02-1976/05): Beatty et al. 1985 [390]

- $\quad$ IMP8 (1976/06-1977/04): Beatty et al. 1985 [390]

- $\quad$ IMP8 (1977/01-1979/12): Evenson et al. 1983 [392]

- ISEE

- ISEE3-HIST (1978/08-1978/12): Mewaldt et al. 1980, 1981, 1986 [393-396]

- ISEE3-MEH (1978/08-1978/12): Kroeger 1986 [397]

- $\quad$ ISEE3-HKH (1978/08-1979/08): Wiedenbeck and Greiner 1980 [398] 
- $\quad$ ISEE3-HKH (1978/08-1980/05): Wiedenbeck and Greiner 1981 [399,400]

- ISEE3-HKH (1978/08-1981/04): Wiedenbeck 1983 [401], Krombel and Wiedenbeck 1988 [402], Leske 1993 [403]

- $\quad$ ISEE3-MEH (1978/08-1984/04): Kroeger 1986 [397]

- ISEE3-MEH (1979/01-1979/12): Kroeger 1986 [397]

- ISEE3-MEH (1980/01-1980/12): Kroeger 1986 [397]

- ISEE3-MEH (1981/01-1981/12): Kroeger 1986 [397]

- ISEE3-MEH (1982/01-1982/12): Kroeger 1986 [397]

- $\quad$ ISEE3-MEH (1983/01-1983/12): Kroeger 1986 [397]

- $\quad$ ISEE3-MEH (1984/01-1984/12): Kroeger 1986 [397]

- NUCLEON

- NUCLEON (2015/07-2017/06): Grebenyuk et al. 2019 [117]

- NUCLEON-IC (2015/07-2017/06): Grebenyuk et al. 2019 [404]

- NUCLEON-KLEM (2015/07-2017/06): Grebenyuk et al. 2019 [404]

- $\quad$ OGO

- OGO1 (1964/10-1965/11): Comstock et al. 1969 [405]

- OGO1 (1965/03-1965/06): Balasubrahmanyan et al. 1966 [259]

- OGO5 (1968/03-1968/10): Teegarden et al. 1970 [406]

- PAMELA

- PAMELA (2006/07-2006/07): Adriani et al. 2013 [112]

- PAMELA (2006/07-2006/08): Adriani et al. 2013 [112]

- PAMELA (2006/07-2008/03): Adriani et al. 2014 [407]

- PAMELA (2006/07-2008/06): Adriani et al. 2009 [150]

- PAMELA (2006/07-2008/12): Adriani et al. 2010, 2011 [151,408]

- PAMELA (2006/07-2009/12): Adriani et al. 2013 [152]

- PAMELA (2006/08-2006/09): Adriani et al. 2013 [112]

- PAMELA-CALO (2006/06-2010/01): Adriani et al. 2013 [119]

- PAMELA-CALO (2006/07-2007/12): Adriani et al. 2016 [409]

- PAMELA-CALO (2006/07-2014/09): Menn et al. 2018 [410]

- PAMELA-TOF (2006/07-2007/12): Adriani et al. 2013, 2016 [409,411]

- PAMELA-TOF (2006/07-2014/09): Menn et al. 2018 [410]

- PAMELA time series: Adriani et al. 2013, 2015 [112,226], Martucci et al. 2018 [49]

- $\quad$ Pioneer

- Pioneer8 (1968/04): Lezniak and Webber 1971 [412]

- Pioneer10-HET (1972/03-1973/03): Teegarden et al. 1975 [383]

- Pioneer10-HET (1985/04-1988/11): Webber and McDonald 1994 [413]

- $\quad$ SOKOL (1984/03-1986/01): Ivanenko et al. 1993 [414], Turundaevskiy and Podorozhnyi 2017 [415]

- $\quad$ Trek-MIR (1991/06-1992/12): Westphal et al. 1996 [416]

- Ulysses

- $\quad$ Ulysses-HET (1990/10-1995/07): Duvernois and Thayer 1996 [417], Duvernois et al. 1996 [418], Connell and Simpson 1997 [419], Duvernois 1997 [420], Thayer 1997 [421]

- Ulysses-HET (1990/10-1996/12): Simpson and Connell 1998 [422], Connell 1997 [423]

- Ulysses-HET (1990/10-1997/12): Connell 1998 [424,425], Connell 1999 [426], Binns et al. 2005 [352]

- $\quad$ Voyager 
- Voyager1 (2008/05): Webber and Higbie 2009 [427]

- Voyager1 (2012/09-2012/12): Stone et al. 2013 [113]

- Voyager1 (2012/10-2012/12): Stone et al. 2013 [113]

- Voyager1\&2 (1977/01-1991/12): Lukasiak et al. 1994 [428]

- Voyager1\&2 (1977/01-1993/12): Lukasiak et al. 1994 [429], Webber et al. 1996 [430]

- Voyager1\&2 (1977/01-1996/12): Lukasiak et al. 1997 [431,432], Webber et al. 1997 [433]

- Voyager1\&2 (1977/01-1998/12): Lukasiak 1999 [434]

- Voyager1\&2 (1986/01-1989/12): Lukasiak et al. 1994 [435]

- Voyager1-HET (1977/10-1977/11): Webber and Yushak 1983 [294]

- Voyager1-HET (1994/01-1994/09): Seo and McDonald 1995 [436]

- Voyager1-HET (2012/12-2015/06): Cummings et al. 2016 [235]

- Voyager1-HET-Aend (1977/10-1977/11): Webber and Yushak 1983 [294]

- Voyager1-HET-Aend (2012/12-2015/06): Cummings et al. 2016 [235]

- Voyager1-HET-Bend (2012/12-2014/12): Cummings et al. 2016 [235]

- Voyager1-LET (2012/12-2015/06): Cummings et al. 2016 [235]

- Voyager2 (2019/03-2019/07): Stone et al. 2019 [234]

- Voyager2-HET (1986/01-1987/12): Ferrando et al. 1991 [437]

- Voyager2-HET (1987/01-1987/12): Seo et al. 1994 [438]

\section{Ground}

- H.E.S.S.-LEAnalysis (2004/10-2005/12): Aharonian et al. 2007 [131]

- VERITAS

- VERITAS (2007/09-2009/05): Wissel 2010 [439]

- VERITAS (2009/09-2012/05): Archer et al. 2018 [440]

Appendix B.6. Data for UHCRs $(Z>30)$

Balloon

- $\operatorname{TIGER}(2001 / 12+2003 / 12):$ Rauch et al. 2009 [441]

- SuperTIGER (2012/12-2013/03): Murphy et al. 2016 [65]

Space

- Ariel6 (1979/06-1982/02): Fowler et al. 1987 [63]

- $\quad H E A O 3-H N E(1979 / 10-1980 / 06)$ : Binns et al. 1981 [66]

- HEAO3-HNE (1979/10-1981/01): Binns et al. 1982 [442], Stone et al. 1983 [67]

- HEAO3-HNE (1979/10-1981/01): Binns et al. 1983 [443]

- $\operatorname{HEAO3-HNE~(1979/10-1981/06):~Binns~et~al.~} 1985$ [444]

- HEAO3-HNE (1979/10-1981/18): Binns et al. 1989 [64]

- $\quad$ Trek (1991/06-1994/01): Westphal et al. 1998 [70]

- $\quad$ Trek-Extended (1991/06-1995/11): Weaver and Westphal 2002 [71]

- UHCRE-LDEF (1984/01-1990/01): Domingo et al. 1996 [68], Dutta et al. 2003 [69], Donnelly et al. 2012 [3]

\section{References}

1. Ghelfi, A.; Maurin, D.; Cheminet, A.; Derome, L.; Hubert, G.; Melot, F. Neutron monitors and muon detectors for solar modulation studies: 2. phi time series. Adv. Space Res. 2017, 60, 833-847. [CrossRef]

2. Shen, Z.N.; Qin, G.; Zuo, P.; Wei, F. Modulation of Galactic Cosmic Rays from Helium to Nickel in the Inner Heliosphere. Astrophys. J. 2019, 887, 132. [CrossRef] 
3. Donnelly, J.; Thompson, A.; OŚullivan, D.; Daly, J.; Drury, L.; Domingo, V.; Wenzel, K.P. Actinide and Ultra-Heavy Abundances in the Local Galactic Cosmic Rays: An Analysis of the Results from the LDEF Ultra-Heavy Cosmic-Ray Experiment. Astrophys. J. 2012, 747, 40. [CrossRef]

4. Binns, W.R.; Bose, R.G.; Braun, D.L.; Brandt, T.J.; Daniels, W.M.; Dowkontt, P.F.; Fitzsimmons, S.P.; Hahne, D.J.; Hams, T.; Israel, M.H.; et al. The SUPERTIGER Instrument: Measurement of Elemental Abundances of Ultra-Heavy Galactic Cosmic Rays. Astrophys. J. 2014, 788, 18. [CrossRef]

5. Abbott, B.P.; Abbott, R.; Abbott, T.D.; Acernese, F.; Ackley, K.; Adams, C.; Adams, T.; Addesso, P.; LIGO Scientific Collaboration; Virgo Collaboration; et al. GW170817: Observation of Gravitational Waves from a Binary Neutron Star Inspiral. Phys. Rev. Lett. 2017, 119, 161101. [CrossRef] [PubMed]

6. Nicholl, M.; Berger, E.; Kasen, D.; Metzger, B.D.; Elias, J.; Briceño, C.; Alexander, K.D.; Blanchard, P.K.; Chornock, R.; Cowperthwaite, P.S.; et al. The Electromagnetic Counterpart of the Binary Neutron Star Merger LIGO/Virgo GW170817. III. Optical and UV Spectra of a Blue Kilonova from Fast Polar Ejecta. Astrophys. J. Lett. 2017, 848, L18. [CrossRef]

7. Chornock, R.; Berger, E.; Kasen, D.; Cowperthwaite, P.S.; Nicholl, M.; Villar, V.A.; Alexander, K.D.; Blanchard, P.K.; Eftekhari, T.; Fong, W.; et al. The Electromagnetic Counterpart of the Binary Neutron Star Merger LIGO/Virgo GW170817. IV. Detection of Near-infrared Signatures of r-process Nucleosynthesis with Gemini-South. Astrophys. J. Lett. 2017, 848, L19. [CrossRef]

8. Grenier, I.A.; Black, J.H.; Strong, A.W. The Nine Lives of Cosmic Rays in Galaxies. Annu. Rev. Astron. Astrophys. 2015, 53, 199-246. [CrossRef]

9. Lavalle, J.; Salati, P. Dark matter indirect signatures. Comptes Rendus Phys. 2012, 13, 740-782. [CrossRef]

10. Abe, K.; Fuke, H.; Haino, S.; Hams, T.; Hasegawa, M.; Horikoshi, A.; Itazaki, A.; Kim, K.C.; Kumazawa, T.; Kusumoto, A.; et al. Search for Antihelium with the BESS-Polar Spectrometer. Phys. Rev. Lett. 2012, 108, 131301. [CrossRef]

11. Aramaki, T.; Boggs, S.; Bufalino, S.; Dal, L.; von Doetinchem, P.; Donato, F.; Fornengo, N.; Fuke, H.; Grefe, M.; Hailey, C.; et al. Review of the theoretical and experimental status of dark matter identification with cosmic-ray antideuterons. Phys. Rep. 2016, 618, 1-37. [CrossRef]

12. Ting, S. The First Five Years of the Alpha Magnetic Spectrometer on the ISS. Available online: https:/ /indico.cern.ch/event/592392/ (accessed on 7 July 2020).

13. Aguilar, M.; Aisa, D.; Alpat, B.; Alvino, A.; Ambrosi, G.; Andeen, K.; Arruda, L.; Attig, N.; Azzarello, P.; Bachlechner, A.; et al. Precision Measurement of the Proton Flux in Primary Cosmic Rays from Rigidity 1 GV to 1.8 TV with the Alpha Magnetic Spectrometer on the International Space Station. Phys. Rev. Lett. 2015, 114, 171103. [CrossRef]

14. Aguilar, M.; Aisa, D.; Alpat, B.; Alvino, A.; Ambrosi, G.; Andeen, K.; Arruda, L.; Attig, N.; Azzarello, P.; Bachlechner, A.; et al. Precision Measurement of the Helium Flux in Primary Cosmic Rays of Rigidities 1.9 GV to 3 TV with the Alpha Magnetic Spectrometer on the International Space Station. Phys. Rev. Lett. 2015, 115, 211101. [CrossRef] [PubMed]

15. Aguilar, M.; Ali Cavasonza, L.; Ambrosi, G.; Arruda, L.; Attig, N.; Aupetit, S.; Azzarello, P.; Bachlechner, A.; Barao, F.; Barrau, A.; et al. Observation of New Properties of Secondary Cosmic Rays Lithium, Beryllium, and Boron by the Alpha Magnetic Spectrometer on the International Space Station. Phys. Rev. Lett. 2018, 120, 021101. [CrossRef] [PubMed]

16. Aartsen, M.G.; Ackermann, M.; Adams, J.; Aguilar, J.A.; Ahlers, M.; Ahrens, M.; Alispach, C.; Andeen, K.; Anderson, T.; Ansseau, I.; et al. Cosmic ray spectrum and composition from PeV to EeV using 3 years of data from IceTop and IceCube. Phys. Rev. D 2019, 100, 082002. [CrossRef]

17. Globus, N.; Allard, D.; Parizot, E. A complete model of the cosmic ray spectrum and composition across the Galactic to extragalactic transition. Phys. Rev. D 2015, 92, 021302. [CrossRef]

18. Thoudam, S.; Rachen, J.P.; van Vliet, A.; Achterberg, A.; Buitink, S.; Falcke, H.; Hörand el, J.R. Cosmic-ray energy spectrum and composition up to the ankle: The case for a second Galactic component. Astron. Astrophys. 2016, 595, A33. [CrossRef]

19. Kotera, K.; Olinto, A.V. The Astrophysics of Ultrahigh-Energy Cosmic Rays. Annu. Rev. Astron. Astrophys. 2011, 49, 119-153. [CrossRef]

20. Strong, A.W.; Moskalenko, I.V. A Galactic Cosmic-Ray Database. arXiv 2009, arXiv:0907.0565.

21. Maurin, D. USINE: Semi-analytical models for Galactic cosmic-ray propagation. Comput. Phys. Commun. 2020, 247, 106942. [CrossRef] 
22. Maurin, D.; Melot, F.; Taillet, R. A database of charged cosmic rays. Astron. Astrophys. 2014, 569 , A32. [CrossRef]

23. Di Felice, V.; Pizzolotto, C.; D’Urso, D.; Dari, S.; Navarra, D.; Primavera, R.; Bertucci, B. Looking for cosmic ray data? The ASI Cosmic Ray Database. In Proceedings of the 35th International Cosmic Ray Conference (ICRC2017), Busan, Korea, 10-20 July 2017; Volume 301, p. 1073.

24. Haungs, A.; Kang, D.; Schoo, S.; Wochele, D.; Wochele, J.; Apel, W.D.; Arteaga-Velázquez, J.C.; Bekk, K.; Bertaina, M.; Blümer, J.; et al. The KASCADE Cosmic-ray Data Centre KCDC: Granting open access to astroparticle physics research data. Eur. Phys. J. C 2018, 78, 741. [CrossRef]

25. Lafferty, G.D.; Wyatt, T.R. Where to stick your data points: The treatment of measurements within wide bins. Nucl. Instrum. Methods Phys. Res. A 1995, 355, 541-547. [CrossRef]

26. Derome, L.; Maurin, D.; Salati, P.; Boudaud, M.; Génolini, Y.; Kunzé, P. Fitting B/C cosmic-ray data in the AMS-02 era: A cookbook. Model numerical precision, data covariance matrix of errors, cross-section nuisance parameters, and mock data. Astron. Astrophys. 2019, 627, A158. [CrossRef]

27. Potgieter, M. Solar Modulation of Cosmic Rays. Living Rev. Sol. Phys. 2013, 10, 3. [CrossRef]

28. Gleeson, L.J.; Axford, W.I. Cosmic Rays in the Interplanetary Medium. Astrophys. J. Lett. 1967, 149 , L115. [CrossRef]

29. Gleeson, L.J.; Axford, W.I. Solar Modulation of Galactic Cosmic Rays. Astrophys. J. 1968, 154, 1011. [CrossRef]

30. Perko, J.S. Solar modulation of galactic antiprotons. Astron. Astrophys. 1987, 184, 119-121.

31. Caballero-Lopez, R.A.; Moraal, H. Limitations of the force field equation to describe cosmic ray modulation. J. Geophys. Res. (Space Phys.) 2004, 109, 1101. [CrossRef]

32. Hathaway, D.H. The Solar Cycle. Living Rev. Sol. Phys. 2015, 12, 4. [CrossRef]

33. Kappl, R. SOLARPROP: Charge-sign dependent solar modulation for everyone. Comput. Phys. Commun. 2016, 207, 386-399. [CrossRef]

34. Boschini, M.J.; Della Torre, S.; Gervasi, M.; La Vacca, G.; Rancoita, P.G. Propagation of cosmic rays in heliosphere: The HELMOD model. Adv. Space Res. 2018, 62, 2859-2879. [CrossRef]

35. Usoskin, I.G.; Alanko-Huotari, K.; Kovaltsov, G.A.; Mursula, K. Heliospheric modulation of cosmic rays: Monthly reconstruction for 1951-2004. J. Geophys. Res. (Space Phys.) 2005, 110, 12108. [CrossRef]

36. Usoskin, I.G.; Gil, A.; Kovaltsov, G.A.; Mishev, A.L.; Mikhailov, V.V. Heliospheric modulation of cosmic rays during the neutron monitor era: Calibration using PAMELA data for 2006-2010. J. Geophys. Res. (Space Phys.) 2017, 122, 3875-3887. [CrossRef]

37. Maurin, D.; Cheminet, A.; Derome, L.; Ghelfi, A.; Hubert, G. Neutron monitors and muon detectors for solar modulation studies: Interstellar flux, yield function, and assessment of critical parameters in count rate calculations. Adv. Space Res. 2015, 55, 363-389. [CrossRef]

38. Ghelfi, A.; Barao, F.; Derome, L.; Maurin, D. Non-parametric determination of H and He interstellar fluxes from cosmic-ray data. Astron. Astrophys. 2016, 591, A94. [CrossRef]

39. Parker, E.N. Cosmic-Ray Modulation by Solar Wind. Phys. Rev. 1958, 110, 1445-1449. [CrossRef]

40. Parker, E.N. Dynamical Instability in an Anisotropic Ionized Gas of Low Density. Phys. Rev. 1958, 109, 1874-1876. [CrossRef]

41. L'Heureux, J.; Fan, C.Y.; Meyer, P. The Quiet-Tiem Spectra of Cosmic-Ray Electrons of Energies Between 10 and $200 \mathrm{MeV}$ Observed on OGO-5. Astrophys. J. 1972, 171, 363. [CrossRef]

42. Fisk, L.A.; Axford, W.I. Solar modulation of galactic cosmic rays, 1. J. Geophys. Res. 1969, 74, 4973. [CrossRef]

43. Fisk, L.A. Solar modulation of galactic cosmic rays, 2. J. Geophys. Res. 1971, 76, 221. [CrossRef]

44. Beatty, J.J.; Ficenec, D.J.; Tobias, S.; Mitchell, J.W.; McKee, S.; Nutter, S.; Tarle, G.; Tomasch, A.; Clem, J.; Guzik, T.G.; et al. The cosmic-ray He-3/He-4 ratio from 100 to $1600 \mathrm{MeV} / \mathrm{amu}$. Astrophys. J. 1993, 413, 268-280. [CrossRef]

45. Jokipii, J.R.; Kopriva, D.A. Effects of particle drift on the transport of cosmic rays. III-Numerical models of galactic cosmic-ray modulation. Astrophys. J. 1979, 234, 384-392. [CrossRef]

46. Potgieter, M.S.; Moraal, H. A drift model for the modulation of galactic cosmic rays. Astrophys. J. 1985, 294, 425-440. [CrossRef]

47. Aguilar, M.; Ali Cavasonza, L.; Alpat, B.; Ambrosi, G.; Arruda, L.; Attig, N.; Aupetit, S.; Azzarello, P.; Bachlechner, A.; Barao, F.; et al. Observation of Fine Time Structures in the Cosmic Proton and Helium Fluxes with the Alpha Magnetic Spectrometer on the International Space Station. Phys. Rev. Lett. 2018, 121, 051101. [CrossRef] [PubMed] 
48. Aguilar, M.; Cavasonza, L.A.; Ambrosi, G.; Arruda, L.; Attig, N.; Aupetit, S.; Azzarello, P.; Bachlechner, A.; Barao, F.; Barrau, A.; et al. Observation of Complex Time Structures in the Cosmic-Ray Electron and Positron Fluxes with the Alpha Magnetic Spectrometer on the International Space Station. Phys. Rev. Lett. 2018, 121, 051102. [CrossRef]

49. Martucci, M.; Munini, R.; Boezio, M.; Di Felice, V.; Adriani, O.; Barbarino, G.C.; Bazilevskaya, G.A.; Bellotti, R.; Bongi, M.; Bonvicini, V.; et al. Proton Fluxes Measured by the PAMELA Experiment from the Minimum to the Maximum Solar Activity for Solar Cycle 24. Astrophys. J. Lett. 2018, 854, L2. [CrossRef]

50. Burbidge, E.M.; Burbidge, G.R.; Fowler, W.A.; Hoyle, F. Synthesis of the Elements in Stars. Rev. Mod. Phys. 1957, 29, 547-650. [CrossRef]

51. Fowler, P.H.; Adams, R.A.; Cowen, V.G.; Kidd, J.M. The Charge Spectrum of Very Heavy Cosmic Ray Nuclei. R. Soc. Lond. Proc. Ser. A 1967, 301, 39-45. [CrossRef]

52. Blanford, G.E.; Friedlander, M.W.; Klarmann, J.; Walker, R.M.; Wefel, J.P.; Wells, W.C.; Fleischer, R.L.; Nichols, G.E.; Price, P.B. Observation of Trans-Iron Nuclei in the Primary Cosmic Radiation. Phys. Rev. Lett. 1969, 23, 338-342. [CrossRef]

53. Fowler, P.H.; Clapham, V.M.; Cowen, V.G.; Kidd, J.M.; Moses, R.T. The Charge Spectrum of Very Heavy Cosmic Ray Nuclei. R. Soc. Lond. Proc. Ser. A 1970, 318, 1-43. [CrossRef]

54. O'Sullivan, D.; Price, P.B.; Shirk, E.K.; Fowler, P.H.; Kidd, J.M.; Kobetich, E.J.; Thorne, R. High-Resolution Measurements of Slowing Cosmic Rays from Fe to U. Phys. Rev. Lett. 1971, 26, 463-466. [CrossRef]

55. Price, P.B.; Fowler, P.H.; Kidd, J.M.; Kobetich, E.J.; Fleischer, R.L.; Nichols, G.E. Study of the Charge Spectrum of Extremely Heavy Cosmic Rays Using Combined Plastic Detectors and Nuclear Emulsions. Phys. Rev. D 1971, 3, 815-823. [CrossRef]

56. Arnould, M.; Goriely, S. The p-process of stellar nucleosynthesis: Astrophysics and nuclear physics status. Phys. Rep. 2003, 384, 1-84. [CrossRef]

57. Rauscher, T.; Dauphas, N.; Dillmann, I.; Fröhlich, C.; Fülöp, Z.; Gyürky, G. Constraining the astrophysical origin of the p-nuclei through nuclear physics and meteoritic data. Rep. Prog. Phys. 2013, 76, 066201. [CrossRef]

58. Cameron, A.G.W.; Thielemann, F.K.; Cowan, J.J. s- and r-process contributions to extinct radioactivities. Phys. Rep. 1993, 227, 283-291. [CrossRef]

59. Meyer, B.S. The r-, s-, and p-Processes in Nucleosynthesis. Annu. Rev. Astron. Astrophys. 1994, 32, $153-190$. [CrossRef]

60. Lingenfelter, R.E. The Origin of Cosmic Rays: How Their Composition Defines Their Sources and Sites and the Processes of Their Mixing, Injection, and Acceleration. Astrophys. J. Suppl. Ser. 2019, 245, 30. [CrossRef]

61. Combet, C.; Maurin, D.; Donnelly, J.; Drury, L.; Vangioni-Flam, E. Spallation-dominated propagation of heavy cosmic rays and the Local Interstellar Medium (LISM). Astron. Astrophys. 2005, 435, 151-160. [CrossRef]

62. Fowler, P.H.; Walker, R.N.F.; Masheder, M.R.W.; Moses, R.T.; Worley, A. Ultra-heavy cosmic ray studies with Ariel VI. Nature 1981, 291, 45-47. [CrossRef]

63. Fowler, P.H.; Walker, R.N.F.; Masheder, M.R.W.; Moses, R.T.; Worley, A.; Gay, A.M. Ariel 6 Measurements of the Fluxes of Ultra-heavy Cosmic Rays. Astrophys. J. 1987, 314, 739. [CrossRef]

64. Binns, W.R.; Garrard, T.L.; Gibner, P.S.; Israel, M.H.; Kertzman, M.P.; Klarmann, J.; Newport, B.J.; Stone, E.C.; Waddington, C.J. Abundances of Ultraheavy Elements in the Cosmic Radiation: Results from HEAO 3. Astrophys. J. 1989, 346, 997. [CrossRef]

65. Murphy, R.P.; Sasaki, M.; Binns, W.R.; Brandt, T.J.; Hams, T.; Israel, M.H.; Labrador, A.W.; Link, J.T.; Mewaldt, R.A.; Mitchell, J.W.; et al. Galactic Cosmic Ray Origins and OB Associations: Evidence from SuperTIGER Observations of Elements ${ }_{26}$ Fe through ${ }_{40}$ Zr. Astrophys. J. 2016, 831, 148. [CrossRef]

66. Binns, W.R.; Fickle, R.K.; Waddington, C.J.; Garrard, T.L.; Stone, E.C.; Israel, M.H.; Klarmann, J. Cosmic-ray abundances of elements with atomic number 26 less than or equal to 40 measured on HEAO 3. Astrophys. J. Lett. 1981, 247, L115-L118. [CrossRef]

67. Stone, E.C.; Garrard, T.L.; Krombel, K.E.; Binns, W.R.; Israel, M.H.; Klarmann, J.; Brewster, N.R.; Fickle, R.K.; Waddington, C.J. Cosmic-ray abundances of the even charge elements from ${ }_{50} \mathrm{Snto}{ }_{58} \mathrm{Ce}$ measured on HEAO-3. In Proceedings of the International Cosmic Ray Conference, Bangalore, India, 22 August-3 September 1983; Volume 9, pp. 115-118. 
68. Domingo, C.; Font, J.; Baixeras, C.; Fernández, F. Source abundances of ultra heavy elements derived from UHCRE measurements. Radiat. Meas. 1996, 26, 825-832. [CrossRef]

69. Dutta, A.; Batra, V.; Biswas, S. Abundance of actinides in cosmic radiation. Radiat. Meas. 2003, 36, $287-290$. [CrossRef]

70. Westphal, A.J.; Price, P.B.; Weaver, B.A.; Afanasiev, V.G. Evidence against stellar chromospheric origin of Galactic cosmic rays. Nature 1998, 396, 50-52. [CrossRef]

71. Weaver, B.A.; Westphal, A.J. Extended Analysis of the Trek Ultraheavy Collector. Astrophys. J. 2002, 569, 493-500. [CrossRef]

72. Aleksandrov, A.B.; Bagulya, A.V.; Vladimirov, M.S.; Goncharova, L.A.; Ivliev, A.I.; Kalinina, G.V.; Kashkarov, L.L.; Konovalova, N.S.; Okat'eva, N.M.; Polukhina, N.G.; et al. INSTRUMENTS AND METHODS OF INVESTIGATION Charge spectrum of galactic cosmic ray nuclei as measured in meteorite olivines. Phys. Uspekhi 2010, 53, 805-808. [CrossRef]

73. Bagulya, A.V.; Kashkarov, L.L.; Konovalova, N.S.; Okat'eva, N.M.; Polukhina, N.G.; Starkov, N.I. Search for superheavy elements in galactic cosmic rays. Sov. J. Exp. Theor. Phys. Lett. 2013, 97, 708-719. [CrossRef]

74. Alexeev, V.; Bagulya, A.; Chernyavsky, M.; Gippius, A.; Goncharova, L.; Gorbunov, S.; Gorshenkov, M.; Kalinina, G.; Konovalova, N.; Liu, J.; et al. Charge Spectrum of Heavy and Superheavy Components of Galactic Cosmic Rays: Results of the Olimpiya Experiment. Astrophys. J. 2016, 829, 120. [CrossRef]

75. Komiya, Y.; Shigeyama, T. R-process Element Cosmic Rays from Neutron Star Mergers. Astrophys. J. 2017, 846, 143. [CrossRef]

76. Bogomolov, E.A.; Lubyanaya, N.D.; Romanov, V.A.; Stepanov, S.V.; Shulakova, M.S. A Stratospheric Magnetic Spectrometer Investigation of the Singly Charged Component Spectra and Composition of the Primary and Secondary Cosmic Radiation. In Proceedings of the International Cosmic Ray Conference, Kyoto, Japan, 6-18 August 1979; Volume 1, p. 330.

77. Buffington, A.; Schindler, S.M.; Pennypacker, C.R. A measurement of the cosmic-ray antiproton flux and a search for antihelium. Astrophys. J. 1981, 248, 1179-1193. [CrossRef]

78. Silk, J.; Srednicki, M. Cosmic-Ray Antiprotons as a Probe of a Photino-Dominated Universe. Phys. Rev. Lett. 1984, 53, 624-627. [CrossRef]

79. Aguilar, M.; Ali Cavasonza, L.; Alpat, B.; Ambrosi, G.; Arruda, L.; Attig, N.; Aupetit, S.; Azzarello, P.; Bachlechner, A.; Barao, F.; et al. Antiproton Flux, Antiproton-to-Proton Flux Ratio, and Properties of Elementary Particle Fluxes in Primary Cosmic Rays Measured with the Alpha Magnetic Spectrometer on the International Space Station. Phys. Rev. Lett. 2016, 117, 091103. [CrossRef] [PubMed]

80. Kachelriess, M.; Ostapchenko, S.; Tjemsland, J. Revisiting cosmic ray antinuclei fluxes with a new coalescence mode. arXiv 2020, arXiv:2002.10481.

81. Galaktionov, Y.V. Antimatter in cosmic rays. Rep. Prog. Phys. 2002, 65, 1243-1270. [CrossRef]

82. Poulin, V.; Salati, P.; Cholis, I.; Kamionkowski, M.; Silk, J. Where do the AMS-02 antihelium events come from? Phys. Rev. D 2019, 99, 023016. [CrossRef]

83. Cholis, I.; Linden, T.; Hooper, D. Anti-Deuterons and Anti-Helium Nuclei from Annihilating Dark Matter. arXiv 2020, arXiv:2001.08749.

84. von Doetinchem, P.; Perez, K.; Aramaki, T.; Baker, S.; Barwick, S.; Bird, R.; Boezio, M.; Boggs, S.E.; Cui, M.; Datta, A.; et al. Cosmic-ray Antinuclei as Messengers of New Physics: Status and Outlook for the New Decade. arXiv 2020, arXiv:2002.04163.

85. Adriani, O.; Barbarino, G.C.; Bazilevskaya, G.A.; Bellotti, R.; Boezio, M.; Bogomolov, E.A.; Bongi, M.; Bonvicini, V.; Bottai, S.; Bruno, A.; et al. The PAMELA Mission: Heralding a new era in precision cosmic ray physics. Phys. Rep. 2014, 544, 323-370. [CrossRef]

86. Smoot, G.F.; Buffington, A.; Orth, C.D. Search for Cosmic-Ray Antimatter. Phys. Rev. Lett. 1975, 35, $258-261$. [CrossRef]

87. Badhwar, G.D.; Golden, R.L.; Lacy, J.L.; Zipse, J.E.; Daniel, R.R.; Stephens, S.A. Relative abundance of antiprotons and antihelium in the primary cosmic radiation. Nature 1978, 274, 137-139. [CrossRef]

88. Ormes, J.F.; Moiseev, A.A.; Saeki, T.; Anraku, K.; Orito, S.; Golden, R.L.; Imori, M.; Inaba, S.; Kimbell, B.L.; Kimura, N.; et al. Antihelium in Cosmic Rays: A New Upper Limit and Its Significance. Astrophys. J. Lett. 1997, 482, L187-L190. [CrossRef]

89. Golden, R.L.; Stochaj, S.J.; Stephens, S.A.; Moiseev, A.A.; Ormes, J.F.; Streitmatter, R.E.; Bowen, T.; Moats, A.; Lloyd-Evans, J. Search for Antihelium in the Cosmic Rays. Astrophys. J. 1997, 479, 992-996. [CrossRef] 
90. Saeki, T.; Anraku, K.; Orito, S.; Ormes, J.; Imori, M.; Kimbell, B.; Makida, Y.; Matsumoto, H.; Matsunaga, H.; Mitchell, J.; et al. A new limit on the flux of cosmic antihelium. Phys. Lett. B 1998, 422, 319-324. [CrossRef]

91. Alcaraz, J.; Alvisi, D.; Alpat, B.; Ambrosi, G.; Anderhub, H.; Ao, L.; Arefiev, A.; Azzarello, P.; Babucci, E.; Baldini, L.; et al. Search for antihelium in cosmic rays. Phys. Lett. B 1999, 461, 387-396. [CrossRef]

92. Sasaki, M.; Matsumoto, H.; Nozaki, M.; Saeki, T.; Abe, K.; Anraku, K.; Asaoka, Y.; Fujikawa, M.; Fuke, H.; Imori, M.; et al. Progress in search for antihelium with BESS. Nucl. Phys. B Proc. Suppl. 2002, 113, $202-207$. [CrossRef]

93. Sasaki, M.; Haino, S.; Abe, K.; Fuke, H.; Hams, T.; Kim, K.C.; Lee, M.H.; Makida, Y.; Matsuda, S.; Mitchell, J.W.; et al. Search for antihelium: Progress with BESS. Adv. Space Res. 2008, 42, 450-454. [CrossRef]

94. Mayorov, A.G.; Galper, A.M.; Adriani, O.; Bazilevskaya, G.A.; Barbarino, G.; Bellotti, R.; Boezio, M.; Bogomolov, E.A.; Bonvicini, V.; Bongi, M.; et al. Upper limit on the antihelium flux in primary cosmic rays. Sov. J. Exp. Theor. Phys. Lett. 2011, 93, 628-631. [CrossRef]

95. Fuke, H.; Maeno, T.; Abe, K.; Haino, S.; Makida, Y.; Matsuda, S.; Matsumoto, H.; Mitchell, J.W.; Moiseev, A.A.; Nishimura, J.; et al. Search for Cosmic-Ray Antideuterons. Phys. Rev. Lett. 2005, 95, 081101. [CrossRef] [PubMed]

96. Aizu, H.; Fujimoto, Y.; Hasegawa, S.; Koshiba, M.; Mito, I.; Nishimura, J.; Yokoi, K.; Schein, M. Heavy Nuclei in the Primary Cosmic Radiation at Prince Albert, Canada. II. Phys. Rev. 1961, 121, 1206-1218. [CrossRef]

97. Greenhill, J.G.; Clarke, A.R.; Elliot, H. Search for Anti-matter in Primary Cosmic Rays. Nature 1971, 230, 170-172. [CrossRef]

98. Golden, R.L.; Adams, J.H.; Boykin, W.R.; Denny, C.L.; Marar, T.M.K.; Heckman, H.H.; Lindstrom, P.L. The Rigidity Spectrum of $Z<=3$ Nuclei from 5 GV to over 300 GV. In Proceedings of the 12th International Cosmic Ray Conference (ICRC12), Hobart, Tasmania, Australia, 16-25 August 1971; Volume 1, p. 203.

99. Buffington, A.; Smith, L.H.; Smoot, G.F.; Alvarez, L.W. Search for Antimatter in Primary Cosmic Rays. Nature 1972, 236, 335-338. [CrossRef]

100. Evenson, P. A Search for Antihelium in Primary Cosmic Radiation. Astrophys. J. 1972, 176, 797. [CrossRef]

101. Verma, R.P.; Rengarajan, T.N.; Tandon, S.N.; Damle, S.V.; Pal, Y. Rigidity Spectrum of Helium Nuclei above 17 GV and a Search for High Energy Anti-nuclei in Primary Cosmic Rays. Nat. Phys. Sci. 1972, 240, 135-137. [CrossRef]

102. Golden, R.L.; Adams, J.H.J.; Deney, C.L.; Badhwar, G.D.; Marar, T.M.K.; Heckman, H.H.; Lindstrom, P.J. Rigidity spectrum of $Z$ ensuremathgeq 3 cosmic-ray nuclei in the range 4-285 GV and a search for cosmic antimatter. Astrophys. J. 1974, 192, 747-751. [CrossRef]

103. Cristinziani, M. Antimatter searches with AMS. Nucl. Phys. B Proc. Suppl. 2002, 113, 195-201. [CrossRef]

104. Kampert, K.H.; Unger, M. Measurements of the Cosmic Ray Composition with Air Shower Experiments. Astropart. Phys. 2012, 35, 660-678. [CrossRef]

105. Apel, W.D.; Arteaga-Velázquez, J.C.; Bekk, K.; Bertaina, M.; Blümer, J.; Bozdog, H.; Brancus, B.M.; Cantoni, E.; Chiavassa, A.; Cossavella, F.; et al. KASCADE-Grande Limits on the Isotropic Diffuse Gamma-Ray Flux between $100 \mathrm{TeV}$ and $1 \mathrm{EeV}$. Astrophys. J. 2017, 848. [CrossRef]

106. Aab, A.; Abreu, P.; Aglietta, M.; Ahn, E.J.; Al Samarai, I.; Albuquerque, I.F.M.; Allekotte, I.; Allen, J.; Allison, P.; Almela, A.; et al. Depth of maximum of air-shower profiles at the Pierre Auger Observatory. II. Composition implications. Phys. Rev. D 2014, 90, 122006. [CrossRef]

107. Apel, W.D.; Arteaga-Velazquez, J.C.; Bekk, K.; Bertaina, M.; Blümer, J.; Bozdog, H.; Brancus, I.M.; Buchholz, P.; Cantonid, E.; Chiavassa, A.; et al. The spectrum of high-energy cosmic rays measured with KASCADE-Grande. Astropart. Phys. 2012, 36, 183-194. [CrossRef]

108. Rawlins, K.; IceCube Collaboration. Latest Results on Cosmic Ray Spectrum and Composition from Three Years of IceTop and IceCube. In Proceedings of the 34th International Cosmic Ray Conference (ICRC2015), The Hague, The Netherlands, 30 July-6 August 2015; Volume 34, p. 334.

109. Aab, A.; Abreu, P.; Aglietta, M.; Ahn, E.J.; Al Samarai, I.; Albuquerque, I.F.M.; Allekotte, I.; Allen, J.; Allison, P.; Almela, A.; et al. Depth of maximum of air-shower profiles at the Pierre Auger Observatory. I. Measurements at energies above $10^{17.8} \mathrm{eV}$. Phys. Rev. D 2014, 90, 122005. [CrossRef]

110. Prosin, V.V.; Berezhnev, S.F.; Budnev, N.M.; Chiavassa, A.; Chvalaev, O.A.; Gress, O.A.; Dyachok, A.N.; Epimakhov, S.N.; Karpov, N.I.; Kalmykov, N.N.; et al. Tunka-133: Results of 3 year operation. Nucl. Instrum. Methods Phys. Res. A 2014, 756, 94-101. [CrossRef] 
111. Aguilar, M.; Ali Cavasonza, L.; Ambrosi, G.; Arruda, L.; Attig, N.; Bachlechner, A.; Barao, F.; Barrau, A.; Barrin, L.; Bartoloni, A.; et al. Properties of Cosmic Helium Isotopes Measured by the Alpha Magnetic Spectrometer. Phys. Rev. Lett. 2019, 123, 181102. [CrossRef]

112. Adriani, O.; Barbarino, G.C.; Bazilevskaya, G.A.; Bellotti, R.; Boezio, M.; Bogomolov, E.A.; Bongi, M.; Bonvicini, V.; Borisov, S.; Bottai, S.; et al. Time Dependence of the Proton Flux Measured by PAMELA during the 2006 July-2009 December Solar Minimum. Astrophys. J. 2013, 765, 91. [CrossRef]

113. Stone, E.C.; Cummings, A.C.; McDonald, F.B.; Heikkila, B.C.; Lal, N.; Webber, W.R. Voyager 1 Observes Low-Energy Galactic Cosmic Rays in a Region Depleted of Heliospheric Ions. Science 2013, 341, 150-153. [CrossRef]

114. Panov, A.D.; Adams, J.H.; Ahn, H.S.; Bashinzhagyan, G.L.; Watts, J.W.; Wefel, J.P.; Wu, J.; Ganel, O.; Guzik, T.G.; Zatsepin, V.I.; et al. Energy spectra of abundant nuclei of primary cosmic rays from the data of ATIC-2 experiment: Final results. Bull. Russ. Acad. Sci. Phys. 2009, 73, 564-567. [CrossRef]

115. Adriani, O.; Akaike, Y.; Asano, K.; Asaoka, Y.; Bagliesi, M.G.; Berti, E.; Bigongiari, G.; Binns, W.R.; Bonechi, S.; Bongi, M.; et al. Direct Measurement of the Cosmic-Ray Proton Spectrum from $50 \mathrm{GeV}$ to $10 \mathrm{TeV}$ with the Calorimetric Electron Telescope on the International Space Station. Phys. Rev. Lett. 2019, 122, 181102. [CrossRef]

116. Yoon, Y.S.; Ahn, H.S.; Allison, P.S.; Bagliesi, M.G.; Beatty, J.J.; Bigongiari, G.; Boyle, P.J.; Childers, J.T.; Conklin, N.B.; Coutu, S.; et al. Cosmic-ray Proton and Helium Spectra from the First CREAM Flight. Astrophys. J. 2011, 728, 122. [CrossRef]

117. Grebenyuk, V.; Karmanov, D.; Kovalev, I.; Kudryashov, I.; Kurganov, A.; Panov, A.; Podorozhny, D.; Tkachenko, A.; Tkachev, L.; Turundaevskiy, A.; et al. Energy spectra of abundant cosmic-ray nuclei in the NUCLEON experiment. Adv. Space Res. 2019, 64, 2546-2558. [CrossRef]

118. Derbina, V.A.; Galkin, V.I.; Hareyama, M.; Hirakawa, Y.; Horiuchi, Y.; Ichimura, M.; Inoue, N.; Kamioka, E.; Kobayashi, T.; Kopenkin, V.V.; et al. Cosmic-Ray Spectra and Composition in the Energy Range of 10-1000 TeV per Particle Obtained by the RUNJOB Experiment. Astrophys. J. Lett. 2005, 628, L41-L44. [CrossRef]

119. Adriani, O.; Barbarino, G.C.; Bazilevskaya, G.A.; Boezio, M.; Bogomolov, E.A.; Bonechi, L.; Bongi, M.; Bonvicini, V.; Borisov, S.V.; Bottai, S.; et al. Measurements of cosmic-ray proton and helium spectra with the PAMELA calorimeter. Adv. Space Res. 2013, 51, 219-226. [CrossRef]

120. Schoo, S.; Apel, W.D.; Arteaga-Velázquez, J.C.; Bekk, K.; Bertaina, M.; Blümer, J.; Bozdog, H.; Brancus, I.M.; Cantoni, E.; Chiavassa, A.; et al. The energy spectrum of cosmic rays in the range from $10^{14}$ to $10^{18} \mathrm{eV}$. In Proceedings of the 34th International Cosmic Ray Conference (ICRC2015), The Hague, The Netherlands, 30 July-6 August 2015; Volume 34, p. 263.

121. Aab, A.; Abreu, P.; Aglietta, M.; Ahn, E.J.; Samarai, I.A.; Albuquerque, I.F.M.; Allekotte, I.; Allison, P.; Almela, A.; et al. The Pierre Auger Observatory: Contributions to the 34th International Cosmic Ray Conference (ICRC 2015). arXiv 2015, arXiv:1509.03732.

122. Ivanov, D. TA Spectrum Summary. In Proceedings of the 34th International Cosmic Ray Conference (ICRC2015), The Hague, The Netherlands, 30 July-6 August 2015; Volume 34, p. 349.

123. Aguilar, M.; Ali Cavasonza, L.; Alpat, B.; Ambrosi, G.; Arruda, L.; Attig, N.; Aupetit, S.; Azzarello, P.; Bachlechner, A.; Barao, F.; et al. Observation of the Identical Rigidity Dependence of He, C, and O Cosmic Rays at High Rigidities by the Alpha Magnetic Spectrometer on the International Space Station. Phys. Rev. Lett. 2017, 119, 251101. [CrossRef] [PubMed]

124. Aguilar, M.; Ali Cavasonza, L.; Alpat, B.; Ambrosi, G.; Arruda, L.; Attig, N.; Aupetit, S.; Azzarello, P.; Bachlechner, A.; Barao, F.; et al. Precision Measurement of Cosmic-Ray Nitrogen and its Primary and Secondary Components with the Alpha Magnetic Spectrometer on the International Space Station. Phys. Rev. Lett. 2018, 121, 051103. [CrossRef]

125. Aguilar, M.; Ali Cavasonza, L.; Ambrosi, G.; Arruda, L.; Attig, N.; Barao, F.; Barrin, L.; Bartoloni, A.; Başeğmez-du Pree, S.; Battiston, R.; et al. Properties of Neon, Magnesium, and Silicon Primary Cosmic Rays Results from the Alpha Magnetic Spectrometer. Phys. Rev. Lett. 2020, 124, 211102. [CrossRef]

126. Simpson, J.A. The Cosmic Ray Nucleonic Component: The Invention and Scientific Uses of the Neutron Monitor-(Keynote Lecture). Space Sci. Rev. 2000, 93, 11-32. [CrossRef]

127. Ahlers, M.; Mertsch, P. Origin of small-scale anisotropies in Galactic cosmic rays. Prog. Part. Nucl. Phys. 2017, 94, 184-216. [CrossRef] 
128. IceCube Collaboration. Evidence for High-Energy Extraterrestrial Neutrinos at the IceCube Detector. Science 2013, 342, 1242856. [CrossRef]

129. Génolini, Y.; Maurin, D.; Moskalenko, I.V.; Unger, M. Current status and desired precision of the isotopic production cross sections relevant to astrophysics of cosmic rays: Li, Be, B, C, and N. Phys. Rev. C 2018, 98, 034611. [CrossRef]

130. Lodders, K. Solar System Abundances and Condensation Temperatures of the Elements. Astrophys. J. 2003, 591, 1220-1247. [CrossRef]

131. Aharonian, F.; Akhperjanian, A.G.; Bazer-Bachi, A.R.; Beilicke, M.; Benbow, W.; Berge, D.; Bernlöhr, K.; Boisson, C.; Bolz, O.; Borrel, V.; et al. First ground-based measurement of atmospheric Cherenkov light from cosmic rays. Phys. Rev. D 2007, 75, 042004. [CrossRef]

132. Golden, R.L.; Horan, S.; Mauger, B.G.; Badhwar, G.D.; Lacy, J.L.; Stephens, S.A.; Daniel, R.R.; Zipse, J.E. Evidence for the existence of cosmic-ray antiprotons. Phys. Rev. Lett. 1979, 43, 1196-1199. [CrossRef]

133. Golden, R.L.; Mauger, B.G.; Nunn, S.; Horan, S. Energy Dependence of the P/P Ratio in Cosmic Rays. Astrophys. Lett. 1984, 24, 75.

134. Bogomolov, E.A.; Krutḱkov, S.Y.; Lubyanaya, N.D.; Romanov, V.A.; Shulakova, M.S.; Stepanov, S.V.; Vasilyev, G.I. Galactic Antiproton Spectrum in the 0.2-5 GEV Range. In Proceedings of the International Cosmic Ray Conference, Moscow, Soviet Union, 2-5 August 1987; Volume 2, p. 72.

135. Moiseev, A.; Yoshimura, K.; Ueda, I.; Anraku, K.; Golden, R.; Imori, M.; Inaba, S.; Kimball, B.; Kimura, N.; Makida, Y.; et al. Cosmic-Ray Antiproton Flux in the Energy Range from 200 to $600 \mathrm{MeV}$. Astrophys. J. 1997, 474, 479. [CrossRef]

136. Matsunaga, H.; Orito, S.; Matsumoto, H.; Yoshimura, K.; Moiseev, A.; Anraku, K.; Golden, R.; Imori, M.; Makida, Y.; Mitchell, J.; et al. Measurement of Low-Energy Cosmic-Ray Antiprotons at Solar Minimum. Phys. Rev. Lett. 1998, 81, 4052-4055. [CrossRef]

137. Orito, S.; Maeno, T.; Matsunaga, H.; Abe, K.; Anraku, K.; Asaoka, Y.; Fujikawa, M.; Imori, M.; Ishino, M.; Makida, Y.; et al. Precision Measurement of Cosmic-Ray Antiproton Spectrum. Phys. Rev. Lett. 2000, 84, 1078-1081. [CrossRef]

138. Maeno, T.; Orito, S.; Matsunaga, H.; Abe, K.; Anraku, K.; Asaoka, Y.; Fujikawa, M.; Imori, M.; Makida, Y.; Matsui, N.; et al. Successive measurements of cosmic-ray antiproton spectrum in a positive phase of the solar cycle. Astropart. Phys. 2001, 16, 121-128. [CrossRef]

139. Asaoka, Y.; Shikaze, Y.; Abe, K.; Anraku, K.; Fujikawa, M.; Fuke, H.; Haino, S.; Imori, M.; Izumi, K.; Maeno, T.; et al. Measurements of Cosmic-Ray Low-Energy Antiproton and Proton Spectra in a Transient Period of Solar Field Reversal. Phys. Rev. Lett. 2002, 88, 051101. [CrossRef]

140. Haino, S.; Abe, K.; Fuke, H.; Maeno, T.; Makida, Y.; Matsumoto, H.; Mitchell, J.W.; Moiseev, A.A.; Nishimura, J.; Nozaki, M.; et al. Measurement of cosmic-ray antiproton spectrum with BESS-2002. In Proceedings of the International Cosmic Ray Conference, Pune, India, 3-11 August 2005; Volume 3, p. 13.

141. Abe, K.; Fuke, H.; Haino, S.; Hams, T.; Itazaki, A.; Kim, K.C.; Kumazawa, T.; Lee, M.H.; Makida, Y.; Matsuda, S.; et al. Measurement of the cosmic-ray low-energy antiproton spectrum with the first BESS-Polar Antarctic flight. Phys. Lett. B 2008, 670, 103-108. [CrossRef]

142. Abe, K.; Fuke, H.; Haino, S.; Hams, T.; Hasegawa, M.; Horikoshi, A.; Kim, K.C.; Kusumoto, A.; Lee, M.H.; Makida, Y.; et al. Measurement of the Cosmic-Ray Antiproton Spectrum at Solar Minimum with a Long-Duration Balloon Flight over Antarctica. Phys. Rev. Lett. 2012, 108, 051102. [CrossRef] [PubMed]

143. Boezio, M.; Carlson, P.; Francke, T.; Weber, N.; Suffert, M.; Hof, M.; Menn, W.; Simon, M.; Stephens, S.A.; Bellotti, R.; et al. The Cosmic-Ray Antiproton Flux between 0.62 and $3.19 \mathrm{G} \mathrm{eV}$ Measured Near Solar Minimum Activity. Astrophys. J. 1997, 487, 415. [CrossRef]

144. Boezio, M.; Bonvicini, V.; Schiavon, P.; Vacchi, A.; Zampa, N.; Bergström, D.; Carlson, P.; Francke, T.; Grinstein, S.; Suffert, M.; et al. The Cosmic-Ray Antiproton Flux between 3 and 49 GeV. Astrophys. J. 2001, 561, 787-799. [CrossRef]

145. Beach, A.S.; Beatty, J.J.; Bhattacharyya, A.; Bower, C.; Coutu, S.; Duvernois, M.A.; Labrador, A.W.; McKee, S.; Minnick, S.A.; Müller, D.; et al. Measurement of the Cosmic-Ray Antiproton-to-Proton Abundance Ratio between 4 and $50 \mathrm{GeV}$. Phys. Rev. Lett. 2001, 87, A261101. [CrossRef]

146. Mitchell, J.W.; Barbier, L.M.; Christian, E.R.; Krizmanic, J.F.; Krombel, K.; Ormes, J.F.; Streitmatter, R.E.; Labrador, A.W.; Davis, A.J.; Mewaldt, R.A.; et al. Measurement of 0.25-3.2 GeV Antiprotons in the Cosmic Radiation. Phys. Rev. Lett. 1996, 76, 3057-3060. [CrossRef] 
147. Basini, G. The Flux of Cosmic Ray Antiprotons from 3.7 to $24 \mathrm{GeV}$. In Proceedings of the International Cosmic Ray Conference, Salt Lake City, UT, USA, 17-25 August 1999; Volume 3, p. 77.

148. Hof, M.; Menn, W.; Pfeifer, C.; Simon, M.; Golden, R.L.; Stochaj, S.J.; Stephens, S.A.; Basini, G.; Ricci, M.; Brancaccio, F.M.; et al. Measurement of Cosmic-Ray Antiprotons from 3.7 to $19 \mathrm{GeV}$. Astrophys. J. Lett. 1996, 467, L33. [CrossRef]

149. Aguilar, M.; Alcaraz, J.; Allaby, J.; Alpat, B.; Ambrosi, G.; Anderhub, H.; Ao, L.; Arefiev, A.; Azzarello, P.; Babucci, E.; et al. The Alpha Magnetic Spectrometer (AMS) on the International Space Station: Part IResults from the test flight on the space shuttle. Phys. Rep. 2002, 366, 331-405. [CrossRef]

150. Adriani, O.; Barbarino, G.C.; Bazilevskaya, G.A.; Bellotti, R.; Boezio, M.; Bogomolov, E.A.; Bonechi, L.; Bongi, M.; Bonvicini, V.; Bottai, S.; et al. New Measurement of the Antiproton-to-Proton Flux Ratio up to 100 $\mathrm{GeV}$ in the Cosmic Radiation. Phys. Rev. Lett. 2009, 102, 051101. [CrossRef]

151. Adriani, O.; Barbarino, G.C.; Bazilevskaya, G.A.; Bellotti, R.; Boezio, M.; Bogomolov, E.A.; Bonechi, L.; Bongi, M.; Bonvicini, V.; Borisov, S.; et al. PAMELA Results on the Cosmic-Ray Antiproton Flux from 60 $\mathrm{MeV}$ to $180 \mathrm{GeV}$ in Kinetic Energy. Phys. Rev. Lett. 2010, 105, 121101. [CrossRef]

152. Adriani, O.; Bazilevskaya, G.A.; Barbarino, G.C.; Bellotti, R.; Boezio, M.; Bogomolov, E.A.; Bonvicini, V.; Bongi, M.; Bonechi, L.; Borisov, S.V.; et al. Measurement of the flux of primary cosmic ray antiprotons with energies of $60 \mathrm{MeV}$ to $350 \mathrm{GeV}$ in the PAMELA experiment. Sov. J. Exp. Theor. Phys. Lett. 2013, 96, 621-627. [CrossRef]

153. Daniel, R.R.; Stephens, S.A. Electron Component of the Primary Cosmic Radiation at Energies $>=15 \mathrm{GeV}$. Phys. Rev. Lett. 1965, 15, 769-772. [CrossRef]

154. Freir, P.S.; Waddington, C.J. Electron, Hydrogen Nuclei, and Helium Nuclei Observed in Primary Cosmic Radiation during 1963. J. Geophys. Res. 1965, 70, 5753-5768. [CrossRef]

155. L'Heureux, J. The Primary Cosmic-Ray Electron Spectrum Near Solar Minimum. Astrophys. J. 1967, 148, 399. [CrossRef]

156. Webber, W.R.; Kish, J.; Rockstroh, J.M. Measurements of the Primary Cosmic Ray Electron Spectrum from 1965 to 1972. In Proceedings of the International Cosmic Ray Conference, Denver, CO, USA, 17-30 August 1973; Volume 2, p. 760.

157. Bleeker, J.A.M.; Burger, J.J.; Scheepmaker, A.; Swanenburg, B.N.; Tanaka, Y. A balloon observation of high energy electrons. In Proceedings of the International Cosmic Ray Conference, London, UK, 6-17 September 1965; Volume 1, p. 327.

158. Fanselow, J.L. The Primary Cosmic-Ray Electron Spectrum Between 0.09 and 8.4 BeV in 1965. Astrophys. J. 1968, 152, 783. [CrossRef]

159. Fanselow, J.L.; Hartman, R.C.; Hildebrad, R.H.; Meyer, P. Charge Composition and Energy Spectrum of Primary Cosmic-Ray Electrons. Astrophys. J. 1969, 158, 771. [CrossRef]

160. Beedle, R.E.; Webber, W.R. Measurements of cosmic-ray electrons in the energy range $4 \mathrm{MeV}$ to $6 \mathrm{BeV}$ at $2 \mathrm{~g} / \mathrm{cm}^{2}$ atmospheric depth at Ft. Churchill. Can. J. Phys. Suppl. 1968, 46, 1014. [CrossRef]

161. Anand, K.C.; Daniel, R.R.; Stephens, S.A. Cosmic-Ray Electron Spectrum above 50 BeV and Its Implications for Cosmic-Ray Confinement. Phys. Rev. Lett. 1968, 20, 764-768. [CrossRef]

162. L'Heureux, J.; Meyer, P. The primary cosmic-ray electron spectrum in the energy range from $300 \mathrm{MeV}$ to $4 \mathrm{BeV}$ from 1964 to 1966. Can. J. Phys. Suppl. 1968, 46, 892. [CrossRef]

163. Earl, J.A.; Neely, D.E.; Rygg, T.A. Balloon measurements of the energy spectrum of cosmic electrons between 1 and 25 Gev. J. Geophys. Res. 1972, 77, 1087. [CrossRef]

164. Bleeker, J.A.M.; Burger, J.J.; Deerenberg, A.J.M.; van de Hulst, H.C.; Scheepmaker, A.; Swanenburg, B.N.; Tanaka, Y. Time variations in the cosmic ray electron spectrum above $500 \mathrm{MeV}$. In Proceedings of the International Cosmic Ray Conference, Budapest, Hungary, 25 August-4 September, 1969; Volume 1, p. 209.

165. Danjo, A.; Hayakawa, S.; Makino, F.; Tanaka, Y. Electron component in primary cosmic rays. I. Experiment. Can. J. Phys. 1968, 46, 530. [CrossRef]

166. Israel, M.H.; Vogt, R.E. Flux of Cosmic-Ray Electrons Between 17 and $63 \mathrm{MeV}$. Phys. Rev. Lett. 1968, 20, 1053-1056. [CrossRef]

167. Agrinier, B.; Koechlin, Y.; Parlier, B.; Paul, J.; Vasseur, J.; Boella, G.; Dilworth, C.; Scarsi, L.; Sironi, G.; Russo, A. East-West asymmetry and charge sign ratio of primary cosmic-ray electrons at $8.3 \mathrm{GV}$ rigidity cut-off. Nuovo Cimento Lett. 1969, 1, 53-56. [CrossRef] 
168. Fanselow, J.L.; Hartman, R.C.; Meyer, P.; Schmidt, P.J. The Energy Spectrum of Primary Cosmic Ray Electrons from $2 \mathrm{GeV}$ to $200 \mathrm{GeV}$. Astrophys. Space Sci. 1971, 14, 301-313. [CrossRef]

169. Kobayashi, T.; Komori, Y.; Yoshida, K.; Yanagisawa, K.; Nishimura, J.; Yamagami, T.; Saito, Y.; Tateyama, N.; Yuda, T.; Wilkes, R.J. Observations of High-energy Cosmic-Ray Electrons from $30 \mathrm{GeV}$ to $3 \mathrm{TeV}$ with Emulsion Chambers. Astrophys. J. 2012, 760, 146. [CrossRef]

170. Anand, K.C.; Daniel, R.R.; Stephens, S.A. Final Results on the TIFR Cosmic Ray Electron Spectrum in the Region 10 to $800 \mathrm{GeV}$. In Proceedings of the International Cosmic Ray Conference, Denver, CO, USA, 17-30 August 1973; Volume 1, p. 355.

171. Scheepmaker, A.; Tanaka, Y. Primary Cosmic-Ray Electron Spectrum between 5 and $300 \mathrm{GeV}$ in 1968. Astron. Astrophys. 1971, 11, 53.

172. Fulks, G.J. Solar modulation of galactic cosmic ray electrons, protons, and alphas. J. Geophys. Res. 1975, 80, 1701-1714. [CrossRef]

173. Beuermann, K.P.; Rice, C.J.; Stone, E.C.; Vogt, R.E. Cosmic-Ray Negatron and Positron Spectra Between 12 and $220 \mathrm{MeV}$. Phys. Rev. Lett. 1969, 22, 412-415. [CrossRef]

174. Silverberg, R.F.; Ormes, J.F.; Balasubrahmanyan, V.K. Primary cosmic ray electrons above 10 Gev: Measurements using a thick detector. J. Geophys. Res. 1973, 78, 7165. [CrossRef]

175. Meegan, C.A.; Earl, J.A. The spectrum of cosmic electrons with energies between 6 and $100 \mathrm{GeV}$. Astrophys. J. 1975, 197, 219-233. [CrossRef]

176. Muller, D.; Meyer, P. The Spectrum of Galactic Electrons with Energies Between 10 and 900 GEV. Astrophys. J. 1973, 186, 841-858. [CrossRef]

177. Silverberg, R.F. Measurement of the primary cosmic electron spectrum from 10 to about $250 \mathrm{GeV}$. J. Geophys. Res. 1976, 81, 3944-3952. [CrossRef]

178. Daugherty, J.K.; Hartman, R.C.; Schmidt, P.J. A measurement of cosmic-ray positron and negatron spectra between 50 and 800 MV. Astrophys. J. 1975, 198, 493-505. [CrossRef]

179. Freier, P.; Gilman, C.; Waddington, C.J. Intensity of primary cosmic-ray electrons of energy exceeding $8 \mathrm{GeV}$. Astrophys. J. 1977, 213, 588-598. [CrossRef]

180. Buffington, A.; Orth, C.D.; Smoot, G.F. Measurement of primary cosmic-ray electrons and positrons from 4 to $50 \mathrm{GeV}$. Astrophys. J. 1975, 199, 669-679. [CrossRef]

181. Ishii, C.; Kobayashi, T.; Shigihara, N.; Yokoi, K.; Matsuo, M.; Nishimura, J.; Taira, T.; Niu, K. Observation of High Energy Primary Electrons with Emulsion Chamber. In Proceedings of the International Cosmic Ray Conference, Denver, CO, USA, 17-30 August 1973; Volume 5, p. 3073.

182. Caldwell, J.; Evenson, P.; Jordan, S.; Meyer, P. The Cosmic Ray Electron Spectrum in 1973 and 1974. In Proceedings of the International Cosmic Ray Conference, Munich, Germany, 15-29 August 1975; Volume 3, p. 1000.

183. Caldwell, J.H.; Evenson, P.; Jordan, S.; Meyer, P. The Cosmic Ray Electron Spectra in 1974 and 1975 and the Implications for Solar Modulation. In Proceedings of the International Cosmic Ray Conference, Plovdiv, Bulgaria, 13-26 August 1977; Volume 11, p. 203.

184. Hartman, R.C.; Pellerin, C.J. Cosmic-ray positron and negatron spectra between 20 and $800 \mathrm{MeV}$ measured in 1974. Astrophys. J. 1976, 204, 927-933. [CrossRef]

185. Prince, T.A. The energy spectrum of cosmic ray electrons between 9 and $300 \mathrm{GeV}$. Astrophys. J. 1979, 227, 676-693. [CrossRef]

186. Golden, R.L.; Mauger, B.G.; Badhwar, G.D.; Daniel, R.R.; Lacy, J.L.; Stephens, S.A.; Zipse, J.E. A measurement of the absolute flux of cosmic-ray electrons. Astrophys. J. 1984, 287, 622-632. [CrossRef]

187. Golden, R.L.; Mauger, B.G.; Horan, S.; Stephens, S.A.; Daniel, R.R.; Badhwar, G.D.; Lacy, J.L.; Zipse, J.E. Observation of cosmic ray positrons in the region from 5 to $50 \mathrm{GeV}$. Astron. Astrophys. 1987, 188, 145-154.

188. Evenson, P.; Meyer, P.; Nandkumar, R. The Energy Spectrum of Cosmic Ray Electrons, 5-150 Mev in Late 1978 and Early 1979. In Proceedings of the International Cosmic Ray Conference, Kyoto, Japan, 6-18 August 1979; Volume 1, p. 462.

189. Evenson, P.; Meyer, P. Solar modulation of cosmic ray electrons 1978-1983. J. Geophys. Res. 1984, 89, $2647-2654$. [CrossRef]

190. Tang, K.K. The energy spectrum of electrons and cosmic-ray confinement A new measurement and its interpretation. Astrophys. J. 1984, 278, 881-892. [CrossRef] 
191. Garcia-Munoz, M.; Meyer, P.; Pyle, K.R.; Simpson, J.A.; Evenson, P. The dependence of solar modulation on the sign of the cosmic ray particle charge. J. Geophys. Res. 1986, 91, 2858-2866. [CrossRef]

192. Mueller, D.; Tang, K.K. Cosmic-ray positrons from 10 to $20 \mathrm{GeV}-\mathrm{A}$ balloon-borne measurement using the geomagnetic east-west asymmetry. Astrophys. J. 1987, 312, 183-194. [CrossRef]

193. Evenson, P.; Huber, D.; Patterson, E.T.; Esposito, J.; Clements, D.; Clem, J. Cosmic electron spectra 1987-1994. J. Geophys. Res. 1995, 100, 7873-7875. [CrossRef]

194. Clem, J.M.; Clements, D.P.; Esposito, J.; Evenson, P.; Huber, D.; L'Heureux, J.; Meyer, P.; Constantin, C. Solar Modulation of Cosmic Electrons. Astrophys. J. 1996, 464, 507. [CrossRef]

195. Clem, J.M.; Evenson, P.; Huber, D.; Pyle, R.; Lopate, C.; Simpson, J.A. Charge sign dependence of cosmic ray modulation near a rigidity of 1 GV. J. Geophys. Res. 2000, 105, 23099-23106. [CrossRef]

196. Clem, J.M.; Evenson, P.A. Positron Abundance in Galactic Cosmic Rays. Astrophys. J. 2002, 568, $216-219$. [CrossRef]

197. Clem, J.; Evenson, P. Observations of cosmic ray electrons and positrons during the early stages of the A-magnetic polarity epoch. J. Geophys. Res. (Space Phys.) 2004, 109, 7107. [CrossRef]

198. Clem, J.; Evenson, P. Balloon-borne observations of the galactic positron fraction during solar minimum negative polarity. J. Geophys. Res. (Space Phys.) 2009, 114, 10108. [CrossRef]

199. Chang, J.; Adams, J.H.; Ahn, H.S.; Bashindzhagyan, G.L.; Christl, M.; Ganel, O.; Guzik, T.G.; Isbert, J.; Kim, K.C.; Kuznetsov, E.N.; et al. An excess of cosmic ray electrons at energies of 300-800 GeV. Nature 2008, 456, 362-365. [CrossRef]

200. Yoshida, K.; Torii, S.; Yamagami, T.; Tamura, T.; Kitamura, H.; Chang, J.; Iijima, I.; Kadokura, A.; Kasahara, K.; Katayose, Y.A.E.A. Cosmic-ray electron spectrum above $100 \mathrm{GeV}$ from PPB-BETS experiment in Antarctica. Adv. Space Res. 2008, 42, 1670-1675. [CrossRef]

201. Torii, S.; Tamura, T.; Tateyama, N.; Yoshida, K.; Nishimura, J.; Yamagami, T.; Murakami, H.; Kobayashi, T.; Komori, Y.; Kasahara, K.; et al. The Energy Spectrum of Cosmic-Ray Electrons from 10 to $100 \mathrm{GeV}$ Observed with a Highly Granulated Imaging Calorimeter. Astrophys. J. 2001, 559, 973-984. [CrossRef]

202. Boezio, M.; Carlson, P.; Francke, T.; Weber, N.; Suffert, M.; Hof, M.; Menn, W.; Simon, M.; Stephens, S.A.; Bellotti, R.; et al. The Cosmic-Ray Electron and Positron Spectra Measured at 1 AU during Solar Minimum Activity. Astrophys. J. 2000, 532, 653-669. [CrossRef]

203. Boezio, M.; Barbiellini, G.; Bonvicini, V.; Schiavon, P.; Vacchi, A.; Zampa, N.; Bergström, D.; Carlson, P.; Francke, T.; Grinstein, S.; et al. Measurements of cosmic-ray electrons and positrons by the Wizard/CAPRICE collaboration. Adv. Space Res. 2001, 27, 669-674. [CrossRef]

204. Beatty, J.J.; Bhattacharyya, A.; Bower, C.; Coutu, S.; Duvernois, M.A.; McKee, S.; Minnick, S.A.; Müller, D.; Musser, J.; Nutter, S.; et al. New Measurement of the Cosmic-Ray Positron Fraction from 5 to $15 \mathrm{GeV}$. Phys. Rev. Lett. 2004, 93, 241102. [CrossRef]

205. Barwick, S.W.; Beatty, J.J.; Bower, C.R.; Chaput, C.J.; Coutu, S.; de Nolfo, G.A.; Duvernois, M.A.; Ellithorpe, D.; Ficenec, D.; Knapp, J.; et al. The Energy Spectra and Relative Abundances of Electrons and Positrons in the Galactic Cosmic Radiation. Astrophys. J. 1998, 498, 779. [CrossRef]

206. Barwick, S.W.; Beatty, J.J.; Bhattacharyya, A.; Bower, C.R.; Chaput, C.J.; Coutu, S.; de Nolfo, G.A.; Knapp, J.; Lowder, D.M.; McKee, S.; et al. Measurements of the Cosmic-Ray Positron Fraction from 1 to $50 \mathrm{GeV}$. Astrophys. J. Lett. 1997, 482, L191. [CrossRef]

207. DuVernois, M.A.; Barwick, S.W.; Beatty, J.J.; Bhattacharyya, A.; Bower, C.R.; Chaput, C.J.; Coutu, S.; de Nolfo, G.A.; Lowder, D.M.; McKee, S.; et al. Cosmic-Ray Electrons and Positrons from 1 to $100 \mathrm{GeV}$ : Measurements with HEAT and Their Interpretation. Astrophys. J. 2001, 559, 296-303. [CrossRef]

208. Golden, R.L.; Grimani, C.; Kimbell, B.L.; Stephens, S.A.; Stochaj, S.J.; Webber, W.R.; Basini, G.; Bongiorno, F.; Massimo Brancaccio, F.; Ricci, M.; et al. Observations of cosmic-ray electrons and positrons using an imaging calorimeter. Astrophys. J. 1994, 436, 769-775. [CrossRef]

209. Grimani, C.; Stephens, S.A.; Cafagna, F.S.; Basini, G.; Bellotti, R.; Brunetti, M.T.; Circella, M.; Codino, A.; De Marzo, C.; De Pascale, M.P.; et al. Measurements of the absolute energy spectra of cosmic-ray positrons and electrons above $7 \mathrm{GeV}$. Astron. Astrophys. 2002, 392, 287-294. [CrossRef]

210. Golden, R.L.; Stochaj, S.J.; Stephens, S.A.; Aversa, F.; Barbiellini, G.; Boezio, M.; Bravar, U.; Colavita, A.; Fratnik, F.; Schiavon, P.; et al. Measurement of the Positron to Electron Ratio in Cosmic Rays above $5 \mathrm{GeV}$. Astrophys. J. Lett. 1996, 457, L103. [CrossRef] 
211. Aguilar, M.; Alcaraz, J.; Allaby, J.; Alpat, B.; Ambrosi, G.; Anderhub, H.; Ao, L.; Arefiev, A.; Azzarello, P.; Baldini, L.; et al. Cosmic-ray positron fraction measurement from 1 to $30 \mathrm{GeV}$ with AMS-01. Phys. Lett. B 2007, 646, 145-154. [CrossRef]

212. Alcaraz, J.; Alpat, B.; Ambrosi, G.; Anderhub, H.; Ao, L.; Arefiev, A.; Azzarello, P.; Babucci, E.; Baldini, L.; Basile, M.; et al. Leptons in near earth orbit. Phys. Lett. B 2000, 484, 10-22. [CrossRef]

213. Aguilar, M.; Alberti, G.; Alpat, B.; Alvino, A.; Ambrosi, G.; Andeen, K.; Anderhub, H.; Arruda, L.; Azzarello, P.; Bachlechner, A.; et al. First Result from the Alpha Magnetic Spectrometer on the International Space Station: Precision Measurement of the Positron Fraction in Primary Cosmic Rays of 0.5-350 GeV. Phys. Rev. Lett. 2013, 110, 141102. [CrossRef] [PubMed]

214. Aguilar, M.; Aisa, D.; Alpat, B.; Alvino, A.; Ambrosi, G.; Andeen, K.; Arruda, L.; Attig, N.; Azzarello, P.; Bachlechner, A.; et al. Precision Measurement of the $\left(\mathrm{e}^{+}+\mathrm{e}^{-}\right)$Flux in Primary Cosmic Rays from $0.5 \mathrm{GeV}$ to $1 \mathrm{TeV}$ with the Alpha Magnetic Spectrometer on the International Space Station. Phys. Rev. Lett. 2014, 113, 221102. [CrossRef]

215. Aguilar, M.; Ali Cavasonza, L.; Alpat, B.; Ambrosi, G.; Arruda, L.; Attig, N.; Azzarello, P.; Bachlechner, A.; Barao, F.; Barrau, A.; et al. Towards Understanding the Origin of Cosmic-Ray Electrons. Phys. Rev. Lett. 2019, 122, 101101. [CrossRef]

216. Adriani, O.; Akaike, Y.; Asano, K.; Asaoka, Y.; Bagliesi, M.G.; Bigongiari, G.; Binns, W.R.; Bonechi, S.; Bongi, M.; Brogi, P.; et al. Energy Spectrum of Cosmic-Ray Electron and Positron from $10 \mathrm{GeV}$ to $3 \mathrm{TeV}$ Observed with the Calorimetric Electron Telescope on the International Space Station. Phys. Rev. Lett. 2017, 119, 181101. [CrossRef]

217. Adriani, O.; Akaike, Y.; Asano, K.; Asaoka, Y.; Bagliesi, M.G.; Berti, E.; Bigongiari, G.; Binns, W.R.; Bonechi, S.; Bongi, M.; et al. Extended Measurement of the Cosmic-Ray Electron and Positron Spectrum from $11 \mathrm{GeV}$ to 4.8 TeV with the Calorimetric Electron Telescope on the International Space Station. Phys. Rev. Lett. 2018, 120, 261102. [CrossRef]

218. Ambrosi, G.; An, Q.; Asfand iyarov, R.; Azzarello, P.; Bernardini, P.; Bertucci, B.; Cai, M.S.; Chang, J.; Chen, D.Y.; Chen, H.F. Direct detection of a break in the teraelectronvolt cosmic-ray spectrum of electrons and positrons. Nature 2017, 552, 63-66. [CrossRef]

219. Ackermann, M.; Ajello, M.; Atwood, W.B.; Baldini, L.; Ballet, J.; Barbiellini, G.; Bastieri, D.; Baughman, B.M.; Bechtol, K.; Bellardi, F.; et al. Fermi LAT observations of cosmic-ray electrons from $7 \mathrm{GeV}$ to $1 \mathrm{TeV}$. Phys. Rev. D 2010, 82, 092004. [CrossRef]

220. Abdollahi, S.; Ackermann, M.; Ajello, M.; Atwood, W.B.; Baldini, L.; Barbiellini, G.; Bastieri, D.; Bellazzini, R.; Bloom, E.D.; Bonino, R.; et al. Cosmic-ray electron-positron spectrum from $7 \mathrm{GeV}$ to $2 \mathrm{TeV}$ with the Fermi Large Area Telescope. Phys. Rev. D 2017, 95, 082007. [CrossRef]

221. Cline, T.L.; Ludwig, G.H.; McDonald, F.B. Detection of Interplanetary 3- to 12-MeV Electrons. Phys. Rev. Lett. 1964, 13, 786-789. [CrossRef]

222. Fan, C.Y.; Gloeckler, G.; Simpson, J.A.; Verma, S.D. The Primary Cosmic-Ray Electron Energy Spectrum from 10 TO 40 Mev. Astrophys. J. 1968, 151, 737. [CrossRef]

223. Evenson, P.; Krawczyk, L.; Moses, D.; Meyer, P. The primary cosmic ray electron spectrum 1978-1980. In Proceedings of the International Cosmic Ray Conference, Paris, France, 13-25 July 1981; Volume 10, pp. 77-80.

224. Burger, J.J.; Swanenburg, B.N. The 1972 cosmic ray electron spectrum above 0.5 GeV. J. Geophys. Res. 1974, 79, 1533. [CrossRef]

225. Burger, J.J.; Swanenburg, B.N. Energy dependent time lag in the long-term modulation of cosmic rays. J. Geophys. Res. 1973, 78, 292. [CrossRef]

226. Adriani, O.; Barbarino, G.C.; Bazilevskaya, G.A.; Bellotti, R.; Boezio, M.; Bogomolov, E.A.; Bongi, M.; Bonvicini, V.; Bottai, S.; Bruno, A.; et al. Time Dependence of the $\mathrm{e}^{-}$Flux Measured by PAMELA during the July 2006-December 2009 Solar Minimum. Astrophys. J. 2015, 810, 142. [CrossRef]

227. Adriani, O.; Barbarino, G.C.; Bazilevskaya, G.A.; Bellotti, R.; Boezio, M.; Bogomolov, E.A.; Bonechi, L.; Bongi, M.; Bonvicini, V.; Bottai, S.; et al. An anomalous positron abundance in cosmic rays with energies 1.5-100 GeV. Nature 2009, 458, 607-609. [CrossRef]

228. Adriani, O.; Barbarino, G.C.; Bazilevskaya, G.A.; Bellotti, R.; Boezio, M.; Bogomolov, E.A.; Bonechi, L.; Bongi, M.; Bonvicini, V.; Borisov, S.; et al. A statistical procedure for the identification of positrons in the PAMELA experiment. Astropart. Phys. 2010, 34, 1-11. [CrossRef] 
229. Adriani, O.; Barbarino, G.C.; Bazilevskaya, G.A.; Bellotti, R.; Bianco, A.; Boezio, M.; Bogomolov, E.A.; Bongi, M.; Bonvicini, V.; Bottai, S.; et al. Cosmic-Ray Positron Energy Spectrum Measured by PAMELA. Phys. Rev. Lett. 2013, 111, 081102. [CrossRef]

230. Adriani, O.; Barbarino, G.C.; Bazilevskaya, G.A.; Bellotti, R.; Boezio, M.; Bogomolov, E.A.; Bongi, M.; Bonvicini, V.; Borisov, S.; Bottai, S.; et al. Cosmic-Ray Electron Flux Measured by the PAMELA Experiment between 1 and $625 \mathrm{GeV}$. Phys. Rev. Lett. 2011, 106, 201101. [CrossRef]

231. Webber, W.R.; Lezniak, J.A.; Damle, S.V. Cosmic ray electrons from 0.2 to 8 Mev: Pioneer 8 and 9 measurements of their spectrum, time variations, and interplanetary radial gradient. J. Geophys. Res. 1973, 78, 1487. [CrossRef]

232. Rastoin, C.; Ferrando, P.; Raviart, A.; Ducros, R.; Petrucci, P.O.; Paizis, C.; Kunow, H.; Mueller-Mellin, R.; Sierks, H.; Wibberenz, G. Time and space variations of the Galactic cosmic ray electron spectrum in the 3-D heliosphere explored by Ulysses. Astron. Astrophys. 1996, 307, 981-995.

233. Caballero-Lopez, R.A.; Moraal, H.; McDonald, F.B. The Modulation of Galactic Cosmic-ray Electrons in the Heliosheath. Astrophys. J. 2010, 725, 121-127. [CrossRef]

234. Stone, E.C.; Cummings, A.C.; Heikkila, B.C.; Lal, N. Cosmic ray measurements from Voyager 2 as it crossed into interstellar space. Nat. Astron. 2019, 3, 1013-1018. [CrossRef]

235. Cummings, A.C.; Stone, E.C.; Heikkila, B.C.; Lal, N.; Webber, W.R.; Jóhannesson, G.; Moskalenko, I.V.; Orlando, E.; Porter, T.A. Galactic Cosmic Rays in the Local Interstellar Medium: Voyager 1 Observations and Model Results. Astrophys. J. 2016, 831, 18. [CrossRef]

236. Aharonian, F.; Akhperjanian, A.G.; Barres de Almeida, U.; Bazer-Bachi, A.R.; Becherini, Y.; Behera, B.; Benbow, W.; Bernlöhr, K.; Boisson, C.; Bochow, A.; et al. Energy Spectrum of Cosmic-Ray Electrons at TeV Energies. Phys. Rev. Lett. 2008, 101, 261104. [CrossRef]

237. Aharonian, F.; Akhperjanian, A.G.; Anton, G.; Barres de Almeida, U.; Bazer-Bachi, A.R.; Becherini, Y.; Behera, B.; Bernlöhr, K.; Bochow, A.; Boisson, C.; et al. Probing the ATIC peak in the cosmic-ray electron spectrum with H.E.S.S. Astron. Astrophys. 2009, 508, 561-564. [CrossRef]

238. Fowler, P.H.; Waddington, C.J.; Freier, P.S.; Naugle, J.; Ney, E.P. The low energy end of the cosmic ray spectrum of alpha-particles. Philos. Mag. 1957, 2, 157-175. [CrossRef]

239. Freier, P.S.; Ney, E.P.; Fowler, P.H. Cosmic Rays and the Sunspot Cycle: Primary $\alpha$-Particle Intensity at Sunspot Maximum. Nature 1958, 181, 1319-1321. [CrossRef]

240. McDonald, F.B. Direct Determination of Primary Cosmic-Ray Alpha-Particle Energy Spectrum by New Method. Phys. Rev. 1956, 104, 1723-1729. [CrossRef]

241. McDonald, F.B.; Webber, W.R. Proton Component of the Primary Cosmic Radiation. Phys. Rev. 1959, 115, 194-205. [CrossRef]

242. McDonald, F.B. Study of Geomagnetic Cutoff Energies and Temporal Variation of the Primary Cosmic Radiation. Phys. Rev. 1957, 107, 1386-1395. [CrossRef]

243. Webber, W.R.; McDonald, F.B. Cerenkov Scintillation Counter Measurements of the Intensity and Modulation of Low Rigidity Cosmic Rays and Features of the Geomagnetic Cutoff Rigidity. J. Geophys. Res. 1964, 69, 3097-3114. [CrossRef]

244. McDonald, F.B. Primary Cosmic-Ray Intensity near Solar Maximum. Phys. Rev. 1959, 116, $462-463$. [CrossRef]

245. McDonald, F.B.; Webber, W.R. Changes in the Low-Rigidity Primary Cosmic Radiation during the Large Forbush Decrease of May 12, 1959. J. Geophys. Res. 1960, 65, 767. [CrossRef]

246. Engler, A.; Kaplon, M.F.; Kernan, A.; Klarmann, J.; Fichtel, C.E.; Friedlander, M.W. Primary cosmic-ray $\alpha$-particles I. Il Nuovo Cimento 1961, 19, 1090-1099. [CrossRef]

247. Engler, A.; Foster, F.; Green, T.L.; Mulvey, J. Primary cosmic ray $\alpha$-particles II. Il Nuovo Cimento 1961, 20, 1157-1165. [CrossRef]

248. Meyer, P.; Vogt, R. Primary Cosmic Ray and Solar Protons. II. Phys. Rev. 1963, 129, 2275-2279. [CrossRef]

249. Freier, P.S.; Waddington, C.J. Intensity of 80- to 200-Mev Protons over Fort Churchill on August 26, 1960. J. Geophys. Res. 1965, 70, 2111-2117. [CrossRef]

250. Freier, P.S.; Waddington, C.J. Singly and doubly charged particles in the primary cosmic radiation. J. Geophys. Res. 1968, 73, 4261-4271. [CrossRef]

251. Fichtel, C.E.; Guss, D.E.; Stevenson, G.R.; Waddington, C.J. Cosmic-Ray Hydrogen and Helium Nuclei during a Solar Quiet Time in July 1961. Phys. Rev. 1964, 133, 818-827. [CrossRef] 
252. Fichtel, C.E.; Guss, D.E.; Kniffen, D.A.; Neelakantan, K.A. Modulation of Low Energy Galactic Cosmic Ray Hydrogen and Helium. J. Geophys. Res. 1964, 69, 3293-3295. [CrossRef]

253. O'dell, F.W.; Shapiro, M.M.; Silberberg, R.; Stiller, B. ${ }^{3} \mathrm{He}-{ }^{4} \mathrm{He}$ in the primary cosmic radiation at Fort Churchill 1963. In Proceedings of the International Cosmic Ray Conference, London, UK, 6-17 September 1965; Volume 1, p. 412.

254. Durgaprasad, N.; Fichtel, C.E.; Guss, D.E. Solar Modulation of Cosmic Rays and Its Relationship to Proton and Helium Fluxes, Interstellar Travel, and Interstellar Secondary Production. J. Geophys. Res. 1967, 72, 2765. [CrossRef]

255. Foster, F.; Schrautemeier, B.E. Energy spectrum and geomagnetic cut-off of primary cosmic-ray $\alpha$-particles near 41deg N mag. Nuovo Cimento A Ser. 1967, 47, 189-194. [CrossRef]

256. Anand, K.C.; Daniel, R.R.; Stephens, S.A.; Bhowmik, B.; Krishna, C.S.; Aditya, P.K.; Puri, R.K. Rigidity spectrum of cosmic-ray helium nuclei >= 12 GV. Can. J. Phys. Suppl. 1968, 46, 652. [CrossRef]

257. Bhatia, V.S.; Paruthi, S.; Kainth, G.S. Simultaneous measurements of helium and heavy nuclei fluxes in cosmic rays over Fort Churchill. J. Geophys. Res. 1977, 82, 2419-2422. [CrossRef]

258. Balasubrahmanyan, V.K.; McDonald, F.B. Solar Modulation Effects on the Primary Cosmic Radiation near Solar Minimum. J. Geophys. Res. 1964, 69, 3289-3292. [CrossRef]

259. Balasubrahmanyan, V.K.; Hagge, D.E.; Ludwig, G.H.; McDonald, F.B. The Multiply Charged Primary Cosmic Radiation at Solar Minimum 1965. J. Geophys. Res. 1966, 71, 1771. [CrossRef]

260. Ormes, J.; Webber, W.R. Measurements of Low-Energy Protons and Alpha Particles in the Cosmic Radiation. Phys. Rev. Lett. 1964, 13, 106-108. [CrossRef]

261. Ormes, J.F.; Webber, W.R. Proton and helium nuclei cosmic-ray spectra and modulations between 100 and $2000 \mathrm{Mev}$ /nucleon. J. Geophys. Res. 1968, 73, 4231-4245. [CrossRef]

262. Webber, W.R.; Ormes, J.F. Cerenkov-scintillation counter measurements of nuclei heavier than helium in the primary cosmic radiation: 1 . Charge composition and energy spectra between $200 \mathrm{Mev} /$ nucleon and 5 bev/nucleon. J. Geophys. Res. 1967, 72, 5957-5976. [CrossRef]

263. von Rosenvinge, T.T.; Ormes, J.F.; Webber, W.R. Measurements of Cosmic-Ray Li, Be and B Nuclei in the Energy Range $100 \mathrm{MeV} / \mathrm{NUC}$ to $>22 \mathrm{BeV} / \mathrm{NUC}$. Astrophys. Space Sci. 1969, 3, 80-101. [CrossRef]

264. Courtier, G.M.; Lenney, A.D. The flux of the cosmic ray hydrogen and helium nuclei at Kiruna, Sweden. Planet. Space Sci. 1966, 14, 503-518. [CrossRef]

265. Hofmann, D.J.; Winckler, J.R. Isotopic Composition of Low-Energy Helium Nuclei in the Primary Cosmic Radiation at Solar Minimum. Phys. Rev. Lett. 1966, 16, 109-111. [CrossRef]

266. Hofmann, D.J.; Winckler, J.R. The measurement at balloon heights of the low energy hydrogen and helium isotopes in the cosmic radiation at solar minimum, 1965. Planet. Space Sci. 1967, 15, 715-725. [CrossRef]

267. Hartman, R.C. The Energy Dependence of the Positron-Electron Ratio in the Primary Cosmic Radiation in 1965. Astrophys. J. 1967, 150, 371. [CrossRef]

268. Rygg, T.A.; Earl, J.A. Balloon measurements of cosmic ray protons and helium over half a solar cycle 1965-1969. J. Geophys. Res. 1971, 76, 7445. [CrossRef]

269. Rygg, T.A.; Ogallagher, J.J.; Earl, J.A. Modulation of cosmic ray protons and helium nuclei near solar maximum. J. Geophys. Res. 1974, 79, 4127-4137. [CrossRef]

270. Garrard, T.L.; Stone, E.C.; Vogt, R.E. Solar Modulation of Cosmic-Ray Protons and He Nuclei. In Proceedings of the International Cosmic Ray Conference, Denver, CO, USA, 17-30 August 1973; Volume 2, p. 732.

271. Webber, W.R.; Chotkowski, C. A determination of the energy spectrum of extraterrestrial electrons in the energy range 70-2000 Mev. J. Geophys. Res. 1967, 72, 2783-2802. [CrossRef]

272. Badhwar, G.D.; Deney, C.L.; Dennis, B.R.; Kaplon, M.F. Measurements of the Low-Energy Cosmic Radiation during the Summer of 1966. Phys. Rev. 1967, 163, 1327-1342. [CrossRef]

273. Behrnetz, S.; Kristiansson, K.; Lindstam, S.; Soderstrom, K. Composition of medium energy cosmic rays from silicon to nickel measured with nuclear emulsion. Astron. Astrophys. 1976, 52, 327-335.

274. Singh, G.; Bhatia, V.S. Charge composition of medium energy cosmic-ray nuclei from neon to iron. Astrophys. Space Sci. 1979, 62, 465-475. [CrossRef]

275. Bjarle, C.; Herrström, N.Y.; Jonsson, G.; Kristiansson, K. The cosmic ray boron and carbon isotopic composition measured in nuclear emulsions. Z. Phys. A Hadron. Nucl. 1979, 291, 383-390. [CrossRef]

276. Webber, W.R.; Damle, S.V.; Kish, J. Studies of the chemical composition of cosmic rays with Z $=3-30$ at high and low energies. Astrophys. Space Sci. 1972, 15, 245-271. [CrossRef] 
277. Ryan, M.J.; Ormes, J.F.; Balasubrahmanyan, V.K. Cosmic-Ray Proton and Helium Spectra above 50 GeV. Phys. Rev. Lett. 1972, 28, 985-988. [CrossRef]

278. Smith, L.H.; Buffington, A.; Smoot, D.F.; Alvarez, L.W.; Wahlig, M.A. A Measurement of Cosmic-Ray Rigidity Spectra above $5 \mathrm{GV} / \mathrm{c}$ of Elements from Hydrogen to Iron. Astrophys. J. 1973, 180, 987-1010. [CrossRef]

279. Apparao, K.M.V. Flux of Cosmic Ray Deuterons with Rigidity Above 16.8 GV. In Proceedings of the International Cosmic Ray Conference, Denver, CO, USA, 17-30 August 1973; Volume 1, p. 126.

280. Juliusson, E. Charge Composition and Energy Spectra of Cosmic-Ray Nuclei at Energies above $20 \mathrm{GeV}$ Per Nucleon. Astrophys. J. 1974, 191, 331-348. [CrossRef]

281. Fisher, A.J.; Hagen, F.A.; Maehl, R.C.; Ormes, J.F.; Arens, J.F. The isotopic composition of cosmic rays with Z between 5 and 26. Astrophys. J. 1976, 205, 938-946. [CrossRef]

282. Hagen, F.A.; Fisher, A.J.; Ormes, J.F. Be-10 abundance and the age of cosmic rays-A balloon measurement. Astrophys. J. 1977, 212, 262-277. [CrossRef]

283. Maehl, R.C.; Ormes, J.F.; Fisher, A.J.; Hagen, F.A. Energy spectra of cosmic ray nuclei-Z of 4 to 26 and E of 0.3 to $2 \mathrm{GeV}$ /amu. Astrophys. Space Sci. 1977, 47, 163-184. [CrossRef]

284. Leech, H.W.; Ogallagher, J.J. The isotopic composition of cosmic-ray helium from 123 to $279 \mathrm{MeV}$ per nucleon-A new measurement and analysis. Astrophys. J. 1978, 221, 1110-1123. [CrossRef]

285. Dwyer, R. The mean mass of the abundant cosmic-ray nuclei from boron to silicon at $1.2 \mathrm{GeV}$ per atomic mass unit. Astrophys. J. 1978, 224, 691-707. [CrossRef]

286. Dwyer, R.; Meyer, P. Cosmic-ray elemental abundances from 1 to $10 \mathrm{GeV}$ per AMU for boron through nickel. Astrophys. J. 1987, 322, 981-991. [CrossRef]

287. Minagawa, G. The abundances and energy spectra of cosmic ray iron and nickel at energies from 1 to $10 \mathrm{GeV}$ per AMU. Astrophys. J. 1981, 248, 847-855. [CrossRef]

288. Scarlett, W.R.; Freier, P.S.; Waddington, C.J. The charge and energy spectra of heavy cosmic-ray nuclei. Astrophys. Space Sci. 1978, 59, 301-311. [CrossRef]

289. Lezniak, J.A.; Webber, W.R. The charge composition and energy spectra of cosmic-ray nuclei from $3000 \mathrm{MeV}$ per nucleon to $50 \mathrm{GeV}$ per nucleon. Astrophys. J. 1978, 223, 676-696. [CrossRef]

290. Derrickson, J.H.; Parnell, T.A.; Austin, R.W.; Selig, W.J.; Gregory, J.C. A measurement of the absolute energy spectra of galactic cosmic rays during the 1976-77 solar minimum. Int. J. Radiat. Appl. Instrum. D Nucl. Tracks Radiat. Meas. 1992, 20, 415-421. [CrossRef]

291. Simon, M.; Spiegelhauer, H.; Schmidt, W.K.H.; Siohan, F.; Ormes, J.F.; Balasubrahmanyan, V.K.; Arens, J.F. Energy spectra of cosmic-ray nuclei to above $100 \mathrm{GeV}$ per nucleon. Astrophys. J. 1980, 239, 712-724. [CrossRef]

292. Webber, W.R.; Golden, R.L.; Stephens, S.A. Cosmic ray proton and helium spectra from 5-200 GV measured with a magnetic spectrometer. In Proceedigns of the 20th International Cosmic-ray Conference, v.1: OG Session: Cosmic Ray Origin and Galactic Phenomena, Moscow, Russia, 2-15 Auguest 1987; Volume 1, pp. 325-328.

293. Buffington, A.; Orth, C.D.; Mast, T.S. A measurement of cosmic-ray beryllium isotopes from 200 to $1500 \mathrm{MeV}$ per nucleon. Astrophys. J. 1978, 226, 355-371. [CrossRef]

294. Webber, W.R.; Yushak, S.M. A measurement of the energy spectra and relative abundance of the cosmic-ray $\mathrm{H}$ and $\mathrm{He}$ isotopes over a broad energy range. Astrophys. J. 1983, 275, 391-404. [CrossRef]

295. Webber, W.R.; Kish, J. Further Studies of the Isotopic Composition of Cosmic Ray Li, Be, and B Nuclei. Implications for the Cosmic Ray Age. In Proceedings of the International Cosmic Ray Conference, Kyoto, Japan, 6-18 August 1979; Volume 1, p. 389.

296. Webber, W.R. The charge and isotopic composition of $Z=6-14$ cosmic ray nuclei at their source. Astrophys. J. 1982, 252, 386-392. [CrossRef]

297. Webber, W.R. The Isotopic Composition of fe and Fragmentation Nuclei with $Z=20-25$. In Proceedings of the International Cosmic Ray Conference, Paris, France, 13-25 July 1981; Volume 2, p. 80.

298. Webber, W.R.; Yushak, S.M. Comparative Energy Spectra of Z=3-8 Nuclei in the Energy Range $200 \mathrm{MeV} / \mathrm{nuc}$ to $3 \mathrm{GeV} /$ nuc. In Proceedings of the International Cosmic Ray Conference, Kyoto, Japan, 6-18 August 1979; Volume 12, p. 51.

299. Jordan, S.P. The isotopic composition of helium in the cosmic radiation above 11 gigavolts. Astrophys. J. 1985, 291, 207-218. [CrossRef] 
300. Webber, W.R.; Kish, J.C.; Schrier, D.A. Cosmic ray charge and energy spectrum measurements using a new large area Cerenkov $\mathrm{X} \mathrm{dE} / \mathrm{dx}$ telescope. In Proceedings of the International Cosmic Ray Conference, San Diego, CA, USA, 11-23 August 1985; Volume 2, pp. 16-19.

301. Webber, W.R.; Kish, J.C.; Schrier, D.A. Cosmic ray isotope measurements with a new Cerenkov X total energy telescope. In Proceedings of the International Cosmic Ray Conference, San Diego, CA, USA, 11-23 August 1985; Volume 2, pp. 88-91.

302. Ichimura, M.; Kogawa, M.; Kuramata, S.; Mito, H.; Murabayashi, T.; Nanjo, H.; Nakamura, T.; Ohba, K.; Ohuchi, T.; Ozawa, T.; et al. Observation of heavy cosmic-ray primaries over the wide energy range from $\sim 100 \mathrm{GeV} /$ particle to $100 \mathrm{TeV} /$ particle: Is the celebrated "knee" actually so prominent? Phys. Rev. D 1993, 48, 1949-1975. [CrossRef] [PubMed]

303. Hatano, Y.; Fukada, Y.; Saito, T.; Oda, H.; Yanagita, T. Relative abundance of ${ }^{3} \mathrm{He}$ and ${ }^{4} \mathrm{He}$ in cosmic rays near 10 GV. Phys. Rev. D 1995, 52, 6219-6223. [CrossRef] [PubMed]

304. Kamioka, E.; Hareyama, M.; Ichimura, M.; Ishihara, Y.; Kobayashi, T.; Komatsu, H.; Kuramata, S.; Maruguchi, K.; Matsutani, H.; Mihashi, A.; et al. Azimuthally controlled observation of heavy cosmic-ray primaries by means of the balloon-borne emulsion chamber. Astropart. Phys. 1997, 6, 155-167. [CrossRef]

305. Bogomolov, E.A.; Vasilyev, G.I.; Yu, S.; Krut'kov, S.; Stepanov, S.V.; Shulakova, M.S. The Deuterium Cosmic Ray Intensity from Balloon Measurement in Energy Range $0.8-1.8 \mathrm{GeV} /$ nucl. In Proceedings of the International Cosmic Ray Conference, Rome, Italy, 28 August-8 September 1995; Volume 2, p. 598.

306. Buckley, J.; Dwyer, J.; Mueller, D.; Swordy, S.; Tang, K.K. A new measurement of the flux of the light cosmic-ray nuclei at high energies. Astrophys. J. 1994, 429, 736-747. [CrossRef]

307. Hesse, A.; Acharya, B.S.; Heinbach, U.; Heinrich, W.; Henkel, M.; Luzietti, B.; Simon, M.; Christian, E.R.; Esposito, J.A.; Balasubrahmanyan, V.K.; et al. Isotopic composition of silicon and iron in the galactic cosmic radiation. Astron. Astrophys. 1996, 314, 785-794.

308. Esposito, J.A.; Christian, E.R.; Balasubrahmanyan, V.K.; Barbier, L.M.; Ormes, J.F.; Streitmatter, R.E.; Acharya, B.; Luzietti, B.; Hesse, A.; Heinbach, U. The ALICE instrument and the measured cosmic ray elemental abundances. Astropart. Phys. 1992, 1, 33-45. [CrossRef]

309. Panov, A.D.; Sokolskaya, N.V.; Adams, J.H., Jr.; Ahn, H.S.; Bashindzhagyan, G.L.; Batkov, K.E.; Chang, J.; Christl, M.; Fazely, A.R.; Gunasingha, R. Relative abundances of cosmic ray nuclei B-C-N-O in the energy region from $10 \mathrm{GeV} / \mathrm{n}$ to $300 \mathrm{GeV} / \mathrm{n}$. Results from ATIC-2 (the science flight of ATIC). In Proceedings of the International Cosmic Ray Conference, Goslar, Germany, 9-11 November 2008; Volume 2, pp. 3-6.

310. Wang, J.Z.; Seo, E.S.; Anraku, K.; Fujikawa, M.; Imori, M.; Maeno, T.; Matsui, N.; Matsunaga, H.; Motoki, M.; Orito, S.; et al. Measurement of Cosmic-Ray Hydrogen and Helium and Their Isotopic Composition with the BESS Experiment. Astrophys. J. 2002, 564, 244-259. [CrossRef]

311. Seo, E.S.; Wang, J.Z.; Matsunaga, H.; Anraku, K.; Imori, M.; Makida, Y.; Matsumoto, H.; McDonald, F.B.; Mitchell, J.; Moiseev, A.A. Spectra of $\mathrm{H}$ and He measured in a series of annual flights. Adv. Space Res. 2001, 26, 1831-1834. [CrossRef]

312. Myers, Z.D.; Seo, E.S.; Abe, K.; Anraku, K.; Imori, M.; Maeno, T.; Makida, Y.; Matsumoto, H.; Mitchell, J.; Moiseev, A.; et al. Cosmic Ray ${ }^{3} \mathrm{He}$ and ${ }^{4} \mathrm{He}$ Spectra from BESS 98. In Proceedings of the International Cosmic Ray Conference, Tsukuba, Japan, 31 July-7 August 2003; Volume 4, p. 1805.

313. Myers, Z.D.; Seo, E.S.; Wang, J.Z.; Alford, R.W.; Abe, K.; Anraku, K.; Asaoka, Y.; Fujikawa, M.; Imori, M.; Maeno, T.A. Cosmic ray ${ }^{1} \mathrm{H}$ and ${ }^{2} \mathrm{H}$ spectra from BESS 98. Adv. Space Res. 2005, 35, 151-155. [CrossRef]

314. Kim, K.C.; Abe, K.; Fuke, H.; Hams, T.; Lee, M.H.; Makida, Y.; Matsuda, S.; Mitchell, J.W.; Nishimura, J.; Ormes, J.F.A. Cosmic ray ${ }^{2} \mathrm{H} /{ }^{1} \mathrm{H}$ ratio measured from BESS in 2000 during solar maximum. Adv. Space Res. 2013, 51, 234-237. [CrossRef]

315. Shikaze, Y.; Haino, S.; Abe, K.; Fuke, H.; Hams, T.; Kim, K.C.; Makida, Y.; Matsuda, S.; Mitchell, J.W.; Moiseev, A.A.; et al. Measurements of $0.220 \mathrm{GeV} / \mathrm{n}$ cosmic-ray proton and helium spectra from 1997 through 2002 with the BESS spectrometer. Astropart. Phys. 2007, 28, 154-167. [CrossRef]

316. Sanuki, T.; Motoki, M.; Matsumoto, H.; Seo, E.S.; Wang, J.Z.; Abe, K.; Anraku, K.; Asaoka, Y.; Fujikawa, M.; Imori, M.; et al. Precise Measurement of Cosmic-Ray Proton and Helium Spectra with the BESS Spectrometer. Astrophys. J. 2000, 545, 1135-1142. [CrossRef]

317. Haino, S.; Sanuki, T.; Abe, K.; Anraku, K.; Asaoka, Y.; Fuke, H.; Imori, M.; Itasaki, A.; Maeno, T.; Makida, Y.; et al. Measurements of primary and atmospheric cosmic-ray spectra with the BESS-TeV spectrometer. Phys. Lett. B 2004, 594, 35-46. [CrossRef] 
318. Abe, K.; Fuke, H.; Haino, S.; Hams, T.; Hasegawa, M.; Horikoshi, A.; Itazaki, A.; Kim, K.C.; Kumazawa, T.; Kusumoto, A.A. Measurements of cosmic-ray proton and helium spectra from the BESS-Polar long-duration balloon flights over Antarctica. arXiv 2015, arXiv:1506.01267.

319. Boezio, M.; Carlson, P.; Francke, T.; Weber, N.; Suffert, M.; Hof, M.; Menn, W.; Simon, M.; Stephens, S.A.; Bellotti, R.; et al. The Cosmic-Ray Proton and Helium Spectra between 0.4 and 200 GV. Astrophys. J. 1999, 518, 457-472. [CrossRef]

320. Boezio, M.; Bonvicini, V.; Schiavon, P.; Vacchi, A.; Zampa, N.; Bergström, D.; Carlson, P.; Francke, T.; Hansen, P.; Mocchiutti, E.; et al. The cosmic-ray proton and helium spectra measured with the CAPRICE98 balloon experiment. Astropart. Phys. 2003, 19, 583-604. [CrossRef]

321. Mocchiutti, E.; Wizard/Caprice Collaboration. Measurement of High Energy ${ }^{3} \mathrm{He}$ in Cosmic Rays by the CAPRICE98 Balloon Experiment. In Proceedings of the International Cosmic Ray Conference, Tsukuba, Japan, 31 July- 7 August 2003; Volume 4, p. 1809.

322. Papini, P.; Piccardi, S.; Spillantini, P.; Vannuccini, E.; Ambriola, M.; Bellotti, R.; Cafagna, F.; Ciacio, F.; Circella, M.; De Marzo, C.N.; et al. High-Energy Deuteron Measurement with the CAPRICE98 Experiment. Astrophys. J. 2004, 615, 259-274. [CrossRef]

323. Ahn, H.S.; Allison, P.S.; Bagliesi, M.G.; Beatty, J.J.; Bigongiari, G.; Boyle, P.J.; Brandt, T.J.; Childers, J.T.; Conklin, N.B.; Coutu, S.; et al. Measurements of cosmic-ray secondary nuclei at high energies with the first flight of the CREAM balloon-borne experiment. Astropart. Phys. 2008, 30, 133-141. [CrossRef]

324. Yoon, Y.S.; Anderson, T.; Barrau, A.; Conklin, N.B.; Coutu, S.; Derome, L.; Han, J.H.; Jeon, J.A.; Kim, K.C.; Kim, M.H.; et al. Proton and Helium Spectra from the CREAM-III Flight. Astrophys. J. 2017, 839, 5. [CrossRef]

325. Ahn, H.S.; Allison, P.; Bagliesi, M.G.; Barbier, L.; Beatty, J.J.; Bigongiari, G.; Brandt, T.J.; Childers, J.T.; Conklin, N.B.; Coutu, S.; et al. Energy Spectra of Cosmic-ray Nuclei at High Energies. Astrophys. J. 2009, 707, 593-603. [CrossRef]

326. Ahn, H.S.; Allison, P.S.; Bagliesi, M.G.; Barbier, L.; Beatty, J.J.; Bigongiari, G.; Brandt, T.J.; Childers, J.T.; Conklin, N.B.; Coutu, S.; et al. Measurements of the Relative Abundances of High-energy Cosmic-ray Nuclei in the TeV/Nucleon Region. Astrophys. J. 2010, 715, 1400-1407. [CrossRef]

327. Freier, P.S.; Young, J.S.; Waddington, C.J. The neutron-rich isotopes of cosmic-ray neon and magnesium. Astrophys. J. Lett. 1980, 240, L53-L58. [CrossRef]

328. Young, J.S.; Freier, P.S.; Waddington, C.J.; Brewster, N.R.; Fickle, R.K. The elemental and isotopic composition of cosmic rays-Silicon to nickel. Astrophys. J. 1981, 246, 1014-1030. [CrossRef]

329. Gibner, P.S.; Mewaldt, R.A.; Schindler, S.M.; Stone, E.C.; Webber, W.R. The isotopic composition of cosmic-ray B, C, N, and O-Evidence for an overabundance of O-18. Astrophys. J. Lett. 1992, 391, L89-L92. [CrossRef]

330. Reimer, O.; Menn, W.; Hof, M.; Simon, M.; Davis, A.J.; Labrador, A.W.; Mewaldt, R.A.; Schindler, S.M.; Barbier, L.M.; Christian, E.R.; et al. The Cosmic-Ray 3He/4He Ratio from $200 \mathrm{MeV}$ per Nucleon to $3.7 \mathrm{GeV}$ per Nucleon. Astrophys. J. 1998, 496, 490. [CrossRef]

331. Menn, W.; Hof, M.; Reimer, O.; Simon, M.; Davis, A.J.; Labrador, A.W.; Mewaldt, R.A.; Schindler, S.M.; Barbier, L.M.; Christian, E.R.; et al. The Absolute Flux of Protons and Helium at the Top of the Atmosphere Using IMAX. Astrophys. J. 2000, 533, 281-297. [CrossRef]

332. de Nolfo, G.A.; Barbier, L.M.; Christian, E.R.; Davis, A.J.; Golden, R.L.; Hof, M.; Krombel, K.E.; Labrador, A.W.; Menn, W.; Mewaldt, R.A.; et al. A measurement of cosmic ray deuterium from 0.5-2.9 GeV/nucleon. In Acceleration and Transport of Energetic Particles Observed in the Heliosphere; Mewaldt, R.A., Jokipii, J.R., Lee, M.A., Möbius, E., Zurbuchen, T.H., Eds.; American Institute of Physics Conference Series; American Institute of Physics: College Park, MD, USA, 2000; Volume 528, pp. 425-428. [CrossRef]

333. Tarle, G.; Ahlen, S.P.; Cartwright, B.G. Cosmic ray isotope abundances from chromium to nickel. Astrophys. J. 1979, 230, 607-620. [CrossRef]

334. Tarle, G.; Ahlen, S.P.; Cartwright, B.G.; Solarz, M. A measurement of the cosmic-ray source abundance of Ca-40. Astrophys. J. Lett. 1979, 232, L161-L164. [CrossRef]

335. Hams, T.; Barbier, L.M.; Bremerich, M.; Christian, E.R.; de Nolfo, G.A.; Geier, S.; Göbel, H.; Gupta, S.K.; Hof, M.; Menn, W.; et al. Measurement of the Abundance of Radioactive ${ }^{10} \mathrm{Be}$ and Other Light Isotopes in Cosmic Radiation up to $2 \mathrm{GeV}$ Nucleon $^{-1}$ with the Balloon-borne Instrument ISOMAX. Astrophys. J. 2004, 611, 892-905. [CrossRef] 
336. Asakimori, K.; Burnett, T.H.; Cherry, M.L.; Chevli, K.; Christ, M.J.; Dake, S.; Derrickson, J.H.; Fountain, W.F.; Fuki, M.; Gregory, J.C.; et al. Cosmic-Ray Proton and Helium Spectra: Results from the JACEE Experiment. Astrophys. J. 1998, 502, 278. [CrossRef]

337. Seo, E.S.; Ormes, J.F.; Streitmatter, R.E.; Stochaj, S.J.; Jones, W.V.; Stephens, S.A.; Bowen, T. Measurement of cosmic-ray proton and helium spectra during the 1987 solar minimum. Astrophys. J. 1991, 378, 763-772. [CrossRef]

338. Webber, W.R.; Golden, R.L.; Stochaj, S.J.; Ormes, J.F.; Strittmatter, R.E. A measurement of the cosmic-ray H-2 and He-3 spectra and H-2/He-4 and He-3/He-4 ratios in 1989. Astrophys. J. 1991, 380, 230-234. [CrossRef]

339. Bellotti, R.; Cafagna, F.; Circella, M.; de Marzo, C.N.; Golden, R.L.; Stochaj, S.J.; de Pascale, M.P.; Morselli, A.; Picozza, P.; Stephens, S.A.; et al. Balloon measurements of cosmic ray muon spectra in the atmosphere along with those of primary protons and helium nuclei over midlatitude. Phys. Rev. D 1999, 60, 052002. [CrossRef]

340. Zatsepin, V.I.; Zamchalova, E.A.; Varkovitskaya, A.Y.; Sokolskaya, N.V.; Sazhina, G.P.; Lazareva, T.V. Energy Spectra of Primary Protons and Other Nuclei in Energy Region 10-100 TeV/nucleus. In Proceedings of the International Cosmic Ray Conference, Calgary, AB, Canada, 19-30 July 1993; Volume 2, p. 13.

341. Diehl, E.; Ellithorpe, D.; Müller, D.; Swordy, S.P. The energy spectrum of cosmic-ray protons and helium near $100 \mathrm{GeV}$. Astropart. Phys. 2003, 18, 487-500. [CrossRef]

342. Ahlen, S.P.; Greene, N.R.; Loomba, D.; Mitchell, J.W.; Bower, C.R.; Heinz, R.M.; Mufson, S.L.; Musser, J.; Pitts, J.J.; Spiczak, G.M.; et al. Measurement of the Isotopic Composition of Cosmic-Ray Helium, Lithium, Beryllium, and Boron up to $1700 \mathrm{MEV}$ per Atomic Mass Unit. Astrophys. J. 2000, 534, 757-769. [CrossRef]

343. Wefel, J.P.; Ahlen, S.P.; Beatty, J.J.; Bower, C.R.; Clem, J.; Ficenec, D.J.; Greene, N.; Guzik, T.G.; Heinz, R.M.; Lijowski, M.; et al. Measurements of Cosmic Ray Helium During the 1991 Solar Maximum. In Proceedings of the International Cosmic Ray Conference, Rome, Italy, 28 August-8 September 1995; Volume 2, p. 630.

344. Gahbauer, F.; Hermann, G.; Hörandel, J.R.; Müller, D.; Radu, A.A. A New Measurement of the Intensities of the Heavy Primary Cosmic-Ray Nuclei around $1 \mathrm{TeV}^{\mathrm{amu}}{ }^{-1}$. Astrophys. J. 2004, 607, 333-341. [CrossRef]

345. Ave, M.; Boyle, P.J.; Gahbauer, F.; Höppner, C.; Hörandel, J.R.; Ichimura, M.; Müller, D.; Romero-Wolf, A. Composition of Primary Cosmic-Ray Nuclei at High Energies. Astrophys. J. 2008, 678, 262-273. [CrossRef]

346. Obermeier, A.; Ave, M.; Boyle, P.; Höppner, C.; Hörandel, J.; Müller, D. Energy Spectra of Primary and Secondary Cosmic-Ray Nuclei Measured with TRACER. Astrophys. J. 2011, 742, 14. [CrossRef]

347. George, J.S.; Lave, K.A.; Wiedenbeck, M.E.; Binns, W.R.; Cummings, A.C.; Davis, A.J.; de Nolfo, G.A.; Hink, P.L.; Israel, M.H.; Leske, R.A.; et al. Elemental Composition and Energy Spectra of Galactic Cosmic Rays During Solar Cycle 23. Astrophys. J. 2009, 698, 1666-1681. [CrossRef]

348. Lave, K.A.; Wiedenbeck, M.E.; Binns, W.R.; Christian, E.R.; Cummings, A.C.; Davis, A.J.; de Nolfo, G.A.; Israel, M.H.; Leske, R.A.; Mewaldt, R.A.; et al. Galactic Cosmic-Ray Energy Spectra and Composition during the 2009-2010 Solar Minimum Period. Astrophys. J. 2013, 770, 117. [CrossRef]

349. Wiedenbeck, M.E.; Binns, W.R.; Christian, E.R.; Cummings, A.C.; Dougherty, B.L.; Hink, P.L.; Klarmann, J.; Leske, R.A.; Lijowski, M.; Mewaldt, R.A.; et al. Constraints on the Time Delay between Nucleosynthesis and Cosmic-Ray Acceleration from Observations of ^59NI and ^59CO. Astrophys. J. Lett. 1999, 523, L61-L64. [CrossRef]

350. Yanasak, N.E.; Wiedenbeck, M.E.; Mewaldt, R.A.; Davis, A.J.; Cummings, A.C.; George, J.S.; Leske, R.A.; Stone, E.C.; Christian, E.R.; von Rosenvinge, T.T.; et al. Measurement of the Secondary Radionuclides ${ }^{10} \mathrm{Be}$, ${ }^{26} \mathrm{Al},{ }^{36} \mathrm{Cl},{ }^{54} \mathrm{Mn}$, and ${ }^{14} \mathrm{C}$ and Implications for the Galactic Cosmic-Ray Age. Astrophys. J. 2001, 563, 768-792. [CrossRef]

351. Ogliore, R.C.; Stone, E.C.; Leske, R.A.; Mewaldt, R.A.; Wiedenbeck, M.E.; Binns, W.R.; Israel, M.H.; von Rosenvinge, T.T.; de Nolfo, G.A.; Moskalenko, I.V. The Phosphorus, Sulfur, Argon, and Calcium Isotopic Composition of the Galactic Cosmic Ray Source. Astrophys. J. 2009, 695, 666-678. [CrossRef]

352. Binns, W.R.; Wiedenbeck, M.E.; Arnould, M.; Cummings, A.C.; George, J.S.; Goriely, S.; Israel, M.H.; Leske, R.A.; Mewaldt, R.A.; Meynet, G.; et al. Cosmic-Ray Neon, Wolf-Rayet Stars, and the Superbubble Origin of Galactic Cosmic Rays. Astrophys. J. 2005, 634, 351-364. [CrossRef]

353. Binns, W.R.; Wiedenbeck, M.E.; Christian, E.R.; Cummings, A.C.; George, J.S.; Hink, P.L.; Israel, M.H.; Klarmann, J.; Leske, R.A.; Lijowski, M.; et al. Galactic cosmic ray neon isotopic abundances measured by the cosmic ray isotope spectrometer (cris) on ace. Adv. Space Res. 2001, 27, 767-772. [CrossRef] 
354. de Nolfo, G.A.; Moskalenko, I.V.; Binns, W.R.; Christian, E.R.; Cummings, A.C.; Davis, A.J.; George, J.S.; Hink, P.L.; Israel, M.H.; Leske, R.A.; et al. Observations of the Li, Be, and B isotopes and constraints on cosmic-ray propagation. Adv. Space Res. 2006, 38, 1558-1564. [CrossRef]

355. Alcaraz, J.; Alpat, B.; Ambrosi, G.; Anderhub, H.; Ao, L.; Arefiev, A.; Azzarello, P.; Babucci, E.; Baldini, L.; Basile, M.; et al. Cosmic protons. Phys. Lett. B 2000, 490, 27-35. [CrossRef]

356. Alcaraz, J.; Alpat, B.; Ambrosi, G.; Anderhub, H.; Ao, L.; Arefiev, A.; Azzarello, P.; Babucci, E.; Baldini, L.; Basile, M.; et al. Helium in near Earth orbit. Phys. Lett. B 2000, 494, 193-202. [CrossRef]

357. Xiong, Z.; Chen, H.; Yang, C.; Yang, M.; Chen, G.; Chen, G.; Lü, Y.; Zhuang, H.; Tang, X. Measurement of ${ }^{3} \mathrm{He} /{ }^{4} \mathrm{He}$ ratio in cosmic rays with the AMS experiment. J. High Energy Phys. 2003, 11, 48. [CrossRef]

358. Aguilar, M.; Alcaraz, J.; Allaby, J.; Alpat, B.; Ambrosi, G.; Anderhub, H.; Ao, L.; Arefiev, A.; Arruda, L.; Azzarello, P.; et al. Relative Composition and Energy Spectra of Light Nuclei in Cosmic Rays: Results from AMS-01. Astrophys. J. 2010, 724, 329-340. [CrossRef]

359. Aguilar, M.; Alcaraz, J.; Allaby, J.; Alpat, B.; Ambrosi, G.; Anderhub, H.; Ao, L.; Arefiev, A.; Arruda, L.; Azzarello, P.; et al. Isotopic Composition of Light Nuclei in Cosmic Rays: Results from AMS-01. Astrophys. J. 2011, 736, 105. [CrossRef]

360. Battiston, R. Precision measurements of $\mathrm{e}^{+} \mathrm{e}^{-}$in Cosmic Ray with the Alpha Magnetic Spectrometer on the ISS. Phys. Dark Universe 2014, 4, 6-9. [CrossRef]

361. Aguilar, M.; Ali Cavasonza, L.; Ambrosi, G.; Arruda, L.; Attig, N.; Aupetit, S.; Azzarello, P.; Bachlechner, A.; Barao, F.; Barrau, A.; et al. Precision Measurement of the Boron to Carbon Flux Ratio in Cosmic Rays from 1.9 GV to 2.6 TV with the Alpha Magnetic Spectrometer on the International Space Station. Phys. Rev. Lett. 2016, 117, 231102. [CrossRef]

362. Aguilar, M.; Ali Cavasonza, L.; Ambrosi, G.; Arruda, L.; Attig, N.; Azzarello, P.; Bachlechner, A.; Barao, F.; Barrau, A.; Barrin, L.; et al. Towards Understanding the Origin of Cosmic-Ray Positrons. Phys. Rev. Lett. 2019, 122, 041102. [CrossRef]

363. Swordy, S.P.; Mueller, D.; Meyer, P.; L'Heureux, J.; Grunsfeld, J.M. Relative abundances of secondary and primary cosmic rays at high energies. Astrophys. J. 1990, 349, 625-633. [CrossRef]

364. Mueller, D.; Swordy, S.P.; Meyer, P.; L'Heureux, J.; Grunsfeld, J.M. Energy spectra and composition of primary cosmic rays. Astrophys. J. 1991, 374, 356-365. [CrossRef]

365. Duvernois, M.A.; Garcia-Munoz, M.; Pyle, K.R.; Simpson, J.A.; Thayer, M.R. The Isotopic Composition of Galactic Cosmic-Ray Elements from Carbon to Silicon: The Combined Release and Radiation Effects Satellite Investigation. Astrophys. J. 1996, 466, 457. [CrossRef]

366. Clayton, E.G.; Guzik, T.G.; Wefel, J.P. CRRES Measurements of Energetic Helium During the 1990-1991 Solar Maximum. Sol. Phys. 2000, 195, 175-194. [CrossRef]

367. Stone, E.C. A Measurement of the Primary Proton Flux from 10 to 130 Million Electron Volts. J. Geophys. Res. 1964, 69, 3939-3945. [CrossRef]

368. Marquardt, J.; Heber, B. Galactic cosmic ray hydrogen spectra and radial gradients in the inner heliosphere measured by the HELIOS Experiment 6. Astron. Astrophys. 2019, 625, A153. [CrossRef]

369. Kühl, P.; Gómez-Herrero, R.; Heber, B. Annual Cosmic Ray Spectra from $250 \mathrm{MeV}$ up to $1.6 \mathrm{GeV}$ from 1995-2014 Measured with the Electron Proton Helium Instrument onboard SOHO. Sol. Phys. 2016, 291, 965-974. [CrossRef]

370. Bryant, D.A.; Cline, T.L.; Desai, U.D.; McDonald, F.B. Explorer 12 Observations of Solar Cosmic Rays and Energetic Storm Particles after the Solar Flare of September 28, 1961. J. Geophys. Res. 1962, 67, 4983-5000. [CrossRef]

371. Durgaprasad, N.; Fichtel, C.E.; Guss, D.E.; Reames, D.V.; O'dell, F.W.; Shapiro, M.M.; Silberberg, R.; Stiller, B.; Tsao, C.H. Chemical Composition of Relativistic Cosmic Rays Detected above the Atmosphere. Phys. Rev. D 1970, 1, 1021-1028. [CrossRef]

372. Ferrando, P.; Engelmann, J.J.; Goret, P.; Koch-Miramond, L.; Petrou, N. Measurement of the isotopic composition of cosmic-rays at $3 \mathrm{GeV} / \mathrm{n}$ using a new geomagnetic method. Astron. Astrophys. 1988, 193, 69-80.

373. Engelmann, J.J.; Ferrando, P.; Soutoul, A.; Goret, P.; Juliusson, E. Charge composition and energy spectra of cosmic-ray nuclei for elements from Be to NI-Results from HEAO-3-C2. Astron. Astrophys. 1990, 233, 96-111. 
374. Gloeckler, G. Solar Modulation of the Low-Energy Galactic Helium Spectrum as Observed on the Imp 1 Satellite. J. Geophys. Res. 1965, 70, 5333-5343. [CrossRef]

375. Fan, C.Y.; Gloeckler, G.; Simpson, J.A. Cosmic Radiation Helium Spectrum below 90 Mev per Nucleon Measured on Imp 1 Satellite. J. Geophys. Res. 1965, 70, 3515-3527. [CrossRef]

376. McDonald, F.B.; Ludwig, G.H. Measurement of Low-Energy Primary Cosmic-Ray Protons on IMP-1 Satellite. Phys. Rev. Lett. 1964, 13, 783-785. [CrossRef]

377. Fan, C.Y.; Gloeckler, G.; Hsieh, K.C.; Simpson, J.A. Isotopic Abundances and Energy Spectra of $\mathrm{He}^{3}$ and $\mathrm{He}^{4}$ Above $40 \mathrm{MeV}$ per Nucleon from the Galaxy. Phys. Rev. Lett. 1966, 16, 813-817. [CrossRef]

378. Fan, C.Y.; Gloeckler, G.; Simpson, J.A. Galactic Deuterium and its Energy Spectrum above $20 \mathrm{MeV}$ per Nucleon. Phys. Rev. Lett. 1966, 17, 329-333. [CrossRef]

379. Hsieh, K.C. Study of Solar Modulation of Low-Energy Cosmic Rays Using Differential Spectra of Protons, ${ }^{3} \mathrm{He}$, and ${ }^{4} \mathrm{He}$ at $\mathrm{E}>100 \mathrm{MeV}$ Per Nucleon during the Quiet Time in 1965 and 1967. Astrophys. J. 1970, $159,61$. [CrossRef]

380. Hsieh, K.C.; Mason, G.M.; Simpson, J.A. Cosmic-Ray ${ }^{2}$ H from Satellite Measurements, $1965-1969$. Astrophys. J. 1971, 166, 221. [CrossRef]

381. Mason, G.M. Interstellar Propagation of Galactic Cosmic-Ray Nuclei $2<=\mathrm{Z}<=8$ IN the Energy Range 10-1000 MeV Per Nucleon. Astrophys. J. 1972, 171, 139. [CrossRef]

382. Garcia-Munoz, M.; Mason, G.M.; Simpson, J.A. The anomalous He-4 component in the cosmic-ray spectrum at below approximately $50 \mathrm{MeV}$ per nucleon during 1972-1974. Astrophys. J. 1975, 202, 265-275. [CrossRef]

383. Teegarden, B.J.; von Rosenvinge, T.T.; McDonald, F.B.; Trainor, J.H.; Webber, W.R. Measurement of the fluxes of galactic cosmic-ray H-2 and He-3 in 1972-1973. Astrophys. J. 1975, 202, 815-822. [CrossRef]

384. Garcia-Munoz, M.; Simpson, J.A.; Wefel, J.P. The isotopes of neon in the galactic cosmic rays. Astrophys. J. Lett. 1979, 232, L95-L99. [CrossRef]

385. Mewaldt, R.A.; Stone, E.C.; Vogt, R.E. The isotopic composition of hydrogen and helium in low-energy cosmic rays. Astrophys. J. 1976, 206, 616-621. [CrossRef]

386. Garcia-Munoz, M.; Mason, G.M.; Simpson, J.A. The age of the galactic cosmic rays derived from the abundance of Be-10. Astrophys. J. 1977, 217, 859-877. [CrossRef]

387. Garcia-Munoz, M.; Mason, G.M.; Simpson, J.A. The isotopic composition of galactic cosmic ray lithium, beryllium and boron. In Proceedings of the International Cosmic Ray Conference, Plovdiv, Bulgaria, 13-26 August 1977; Volume 1, pp. 301-306.

388. Guzik, T.G. The low-energy galactic cosmic ray carbon, nitrogen, and oxygen isotopic composition. Astrophys. J. 1981, 244, 695-710. [CrossRef]

389. Garcia-Munoz, M.; Simpson, J.A.; Wefel, J.P. The propagation lifetime of galactic cosmic rays determined from the measurement of the beryllium isotopes. In Proceedings of the International Cosmic Ray Conference, Paris, France, 13-25 July 1981; Volume 2, pp. 72-75.

390. Beatty, J.J.; Garcia-Munoz, M.; Simpson, J.A. The cosmic-ray spectra of H-1, H-2, and He-4 as a test of the origin of the hydrogen superfluxes at solar minimum modulation. Astrophys. J. 1985, 294, 455-462. [CrossRef]

391. Garcia-Munoz, M.; Simpson, J.A.; Guzik, T.G.; Wefel, J.P.; Margolis, S.H. Cosmic-ray propagation in the Galaxy and in the heliosphere-The path-length distribution at low energy. Astrophys. J. Suppl. Ser. 1987, 64, 269-304. [CrossRef]

392. Evenson, P.; Garcia-Munoz, M.; Meyer, P.; Pyle, K.R.; Simpson, J.A. A quantitative test of solar modulation theory-The proton, helium, and electron spectra from 1965 through 1979. Astrophys. J. Lett. 1983, 275, L15-L18. [CrossRef]

393. Mewaldt, R.A.; Spalding, J.D.; Stone, E.C.; Vogt, R.E. The isotopic composition of galactic cosmic-ray iron nuclei. Astrophys. J. Lett. 1980, 236, L121-L125. [CrossRef]

394. Mewaldt, R.A.; Spalding, J.D.; Stone, E.C.; Vogt, R.E. High resolution measurements of galactic cosmic-ray neon, magnesium, and silicon isotopes. Astrophys. J. Lett. 1980, 235, L95-L99. [CrossRef]

395. Mewaldt, R.A.; Spalding, J.D.; Stone, E.C.; Vogt, R.E. The isotropic composition of cosmic ray B, C, N, and O nuclei. Astrophys. J. Lett. 1981, 251, L27-L31. [CrossRef]

396. Mewaldt, R.A. He-3 in galactic cosmic rays. Astrophys. J. 1986, 311, 979-983. [CrossRef]

397. Kroeger, R. Measurements of hydrogen and helium isotopes in Galactic cosmic rays from 1978 through 1984. Astrophys. J. 1986, 303, 816-828. [CrossRef] 
398. Wiedenbeck, M.E.; Greiner, D.E. A cosmic-ray age based on the abundance of Be-10. Astrophys. J. Lett. 1980, 239, L139-L142. [CrossRef]

399. Wiedenbeck, M.E.; Greiner, D.E. High-resolution observations of the isotopic composition of carbon and silicon in the galactic cosmic rays. Astrophys. J. Lett. 1981, 247, L119-L122. [CrossRef]

400. Wiedenbeck, M.E.; Greiner, D.E. Isotopic anomalies in the galactic cosmic-ray source. Phys. Rev. Lett. 1981, 46, 682-685. [CrossRef]

401. Wiedenbeck, M.E. The abundance of the radioactive isotope Al-26 in galactic cosmic rays. In Proceedings of the International Cosmic Ray Conference, Bangalore, India, 22 August-3 September 1983; Volume 9, pp. 147-150.

402. Krombel, K.E.; Wiedenbeck, M.E. Isotopic composition of cosmic-ray boron and nitrogen. Astrophys. J. 1988, 328, 940-953. [CrossRef]

403. Leske, R.A. The elemental and isotopic composition of Galactic cosmic-ray nuclei from scandium through nickel. Astrophys. J. 1993, 405, 567-583. [CrossRef]

404. Grebenyuk, V.; Karmanov, D.; Kovalev, I.; Kudryashov, I.; Kurganov, A.; Panov, A.; Podorozhny, D.; Tkachenko, A.; Tkachev, L.; Turundaevskiy, A.; et al. Secondary cosmic rays in the NUCLEON space experiment. Adv. Space Res. 2019, 64, 2559-2563. [CrossRef]

405. Comstock, G.M.; Fan, C.Y.; Simpson, J.A. Energy Spectra and Abundances of the Cosmic-Ray Nuclei Helium to Iron from the Ogo-I Satellite Experiment. Astrophys. J. 1969, 155, 609. [CrossRef]

406. Teegarden, B.J.; McDonald, F.B.; Balasubrahmanyan, V.K. Spectra and charge composition of the low energy galactic cosmic radiation from $\mathrm{Z}=2$ to 14 . In Proceedings of the International Conference on Cosmic Rays, Budapest, Hungary, 25 August-4 September 1969; Volume 1, p. 345.

407. Adriani, O.; Barbarino, G.C.; Bazilevskaya, G.A.; Bellotti, R.; Boezio, M.; Bogomolov, E.A.; Bongi, M.; Bonvicini, V.; Bottai, S.; Bruno, A.; et al. Measurement of Boron and Carbon Fluxes in Cosmic Rays with the PAMELA Experiment. Astrophys. J. 2014, 791, 93. [CrossRef]

408. Adriani, O.; Barbarino, G.C.; Bazilevskaya, G.A.; Bellotti, R.; Boezio, M.; Bogomolov, E.A.; Bonechi, L.; Bongi, M.; Bonvicini, V.; Borisov, S.; et al. PAMELA Measurements of Cosmic-Ray Proton and Helium Spectra. Science 2011, 332, 69. [CrossRef]

409. Adriani, O.; Barbarino, G.C.; Bazilevskaya, G.A.; Bellotti, R.; Boezio, M.; Bogomolov, E.A.; Bongi, M.; Bonvicini, V.; Bottai, S.; Bruno, A.; et al. Measurements of Cosmic-Ray Hydrogen and Helium Isotopes with the PAMELA Experiment. Astrophys. J. 2016, 818, 68. [CrossRef]

410. Menn, W.; Bogomolov, E.A.; Simon, M.; Vasilyev, G.; Adriani, O.; Barbarino, G.C.; Bazilevskaya, G.A.; Bellotti, R.; Boezio, M.; Bongi, M.; et al. Lithium and Beryllium Isotopes with the PAMELA Experiment. Astrophys. J. 2018, 862, 141. [CrossRef]

411. Adriani, O.; Barbarino, G.C.; Bazilevskaya, G.A.; Bellotti, R.; Boezio, M.; Bogomolov, E.A.; Bongi, M.; Bonvicini, V.; Borisov, S.; Bottai, S.; et al. Measurement of the Isotopic Composition of Hydrogen and Helium Nuclei in Cosmic Rays with the PAMELA Experiment. Astrophys. J. 2013, 770, 2. [CrossRef]

412. Lezniak, J.A.; Webber, W.R. Solar modulation of cosmic ray protons, helium nuclei, and electrons: A comparison of experiment with theory. J. Geophys. Res. 1971, 76, 1605. [CrossRef]

413. Webber, W.R.; McDonald, F.B. The cosmic-ray oxygen and helium spectra measured at Pioneer 10 over the time of the 1987 modulation minimum, and implications for the He/O source ratio. Astrophys. J. 1994, 435, 464-468. [CrossRef]

414. Ivanenko, I.P.; Shestoperov, V.Y.; Chikova, L.O.; Fateeva, I.M.; Khein, L.A.; Podoroznyi, D.M.; Rapoport, I.D.; Samsonov, G.A.; Sobinyakov, V.A.; Turundaevskyi, A.N.; et al. Energy Spectra of Cosmic Rays above $2 \mathrm{TeV}$ as Measured by the 'SOKOL' Apparatus. In Proceedings of the International Cosmic Ray Conference, Calgary, AB, Canada, 19-30 July 1993; Volume 2, p. 17.

415. Turundaevskiy, A.; Podorozhnyi, D. High energy deuterons in cosmic rays registered by the SOKOL satellite experiment. Adv. Space Res. 2017, 59, 496-501. [CrossRef]

416. Westphal, A.J.; Afanasyev, V.G.; Price, P.B.; Solarz, M.; Akimov, V.V.; Rodin, V.G.; Shvets, N.I. Measurement of the Isotopic Composition of Manganese, Iron, and Nickel in the Galactic Cosmic Rays. Astrophys. J. 1996, 468, 679. [CrossRef]

417. Duvernois, M.A.; Thayer, M.R. The Elemental Composition of the Galactic Cosmic-Ray Source: ULYSSES High-Energy Telescope Results. Astrophys. J. 1996, 465, 982. [CrossRef] 
418. Duvernois, M.A.; Simpson, J.A.; Thayer, M.R. Interstellar propagation of cosmic rays: Analysis of the ULYSSES primary and secondary elemental abundances. Astron. Astrophys. 1996, 316, 555-563.

419. Connell, J.J.; Simpson, J.A. Isotopic Abundances of $\mathrm{Fe}$ and $\mathrm{Ni}$ in Galactic Cosmic-Ray Sources. Astrophys. J. Lett. 1997, 475, L61. [CrossRef]

420. Duvernois, M.A. Galactic Cosmic-Ray Manganese: ULYSSES High Energy Telescope Results. Astrophys. J. 1997, 481, 241. [CrossRef]

421. Thayer, M.R. An Investigation into Sulfur Isotopes in the Galactic Cosmic Rays. Astrophys. J. 1997, $482,792$. [CrossRef]

422. Simpson, J.A.; Connell, J.J. Cosmic-Ray 26 A(l and Its Decay in the Galaxy. Astrophys. J. Lett. 1998, 497 , L85. [CrossRef]

423. Connell, J. High Resolution Measurements of the Isotopic Composition of Galactic Cosmic Ray C, N, O, $\mathrm{Ne}, \mathrm{Mg}$ and Si from the Ulysses HET. In Proceedings of the 25th International Conference on Cosmic Rays, Durban, South Africa, 28 July-8 August 1997; Volume 3, p. 381.

424. Connell, J.J. Galactic Cosmic-Ray Confinement Time: ULYSSES High Energy Telescope Measurements of the Secondary Radionuclide 10Be. Astrophys. J. Lett. 1998, 501, L59. [CrossRef]

425. Connell, J.J.; Duvernois, M.A.; Simpson, J.A. The Cosmic-Ray Radioactive Nuclide ${ }^{36}$ CL and Its Propagation in the Galaxy. Astrophys. J. Lett. 1998, 509, L97-L100. [CrossRef]

426. Connell, J. Ulysses HET Measurements of Electron-capture Secondary Isotopes: Testing the Role of Cosmic Ray Reacceleration. In Proceedings of the International Cosmic Ray Conference, Salt Lake City, UT, USA, 17-25 August 1999; Volume 3, p. 33.

427. Webber, W.R.; Higbie, P.R. Galactic propagation of cosmic ray nuclei in a model with an increasing diffusion coefficient at low rigidities: A comparison of the new interstellar spectra with Voyager data in the outer heliosphere. J. Geophys. Res. (Space Phys.) 2009, 114, 2103. [CrossRef]

428. Lukasiak, A.; Ferrando, P.; McDonald, F.B.; Webber, W.R. The isotopic composition of cosmic-ray beryllium and its implication for the cosmic ray's age. Astrophys. J. 1994, 423, 426-431. [CrossRef]

429. Lukasiak, A.; McDonald, F.B.; Webber, W.R. Voyager measurements of the isotopic composition of cosmic-ray aluminum and implications for the propagation of cosmic rays. Astrophys. J. Lett. 1994, 430, L69-L72. [CrossRef]

430. Webber, W.R.; Lukasiak, A.; McDonald, F.B.; Ferrando, P. New High-Statistical-High-Resolution Measurements of the Cosmic-Ray CNO Isotopes from a 17 Year Study Using the Voyager 1 and 2 Spacecraft. Astrophys. J. 1996, 457, 435. [CrossRef]

431. Lukasiak, A.; McDonald, F.B.; Webber, W.R. Voyager Measurements of the Mass Composition of Cosmic-Ray Ca through Fe Nuclei. Astrophys. J. 1997, 488, 454. [CrossRef]

432. Lukasiak, A.; McDonald, F.B.; Webber, W.R. Voyager Measurements of the Isotopic Composition of Li, Be and B Nuclei. In Proceedings of the International Cosmic Ray Conference, Durban, South Africa, 28 July-8 August 1997; Volume 3, p. 389.

433. Webber, W.R.; Lukasiak, A.; McDonald, F.B. Voyager Measurements of the Mass Composition of Cosmic-Ray $\mathrm{Ne}, \mathrm{Mg}, \mathrm{Si}$, and S Nuclei. Astrophys. J. 1997, 476, 766. [CrossRef]

434. Lukasiak, A. Voyager Measurements of the Charge and Isotopic Composition of Cosmic Ray Li, Be and B Nuclei and Implications for Their Production in the Galaxy. In Proceedings of the International Cosmic Ray Conference, Salt Lake City, UT, USA, 17-25 August 1999; Volume 3, p. 41.

435. Lukasiak, A.; Ferrando, P.; McDonald, F.B.; Webber, W.R. Cosmic-ray isotopic composition of C, N, O, Ne, $\mathrm{Mg}$, SI nuclei in the energy range $50-200 \mathrm{MeV}$ per nucleon measured by the Voyager spacecraft during the solar minimum period. Astrophys. J. 1994, 426, 366-372. [CrossRef]

436. Seo, E.S.; McDonald, F.B. Cosmic-Ray H and He Isotopes in the Outer Heliosphere in 1994. Astrophys. J. Lett. 1995, 451, L33. [CrossRef]

437. Ferrando, P.; Lal, N.; McDonald, F.B.; Webber, W.R. Studies of low-energy Galactic cosmic-ray composition at 22 AU. I-Secondary/primary ratios. Astron. Astrophys. 1991, 247, 163-172.

438. Seo, E.S.; McDonald, F.B.; Lal, N.; Webber, W.R. Study of cosmic-ray H and He isotopes at 23 AU. Astrophys. J. 1994, 432, 656-664. [CrossRef]

439. Wissel, S.A. Observations of Direct Cerenkov Light in Ground-Based Telescopes and the Flux of Iron Nuclei at TeV Energies. Ph.D. Thesis, The University of Chicago, Chicago, IL, USA, 2010. 
440. Archer, A.; Benbow, W.; Bird, R.; Brose, R.; Buchovecky, M.; Bugaev, V.; Connolly, M.P.; Cui, W.; Daniel, M.K.; Falcone, A.; et al. Measurement of the iron spectrum in cosmic rays by VERITAS. Phys. Rev. D 2018, 98, 022009. [CrossRef]

441. Rauch, B.F.; Link, J.T.; Lodders, K.; Israel, M.H.; Barbier, L.M.; Binns, W.R.; Christian, E.R.; Cummings, J.R.; de Nolfo, G.A.; Geier, S.; et al. Cosmic Ray origin in OB Associations and Preferential Acceleration of Refractory Elements: Evidence from Abundances of Elements ${ }_{26} \mathrm{Fe}$ through ${ }_{34} \mathrm{Se}$. Astrophys. J. 2009, 697, 2083-2088. [CrossRef]

442. Binns, W.R.; Israel, M.H.; Klarmann, J.; Fickle, R.K.; Waddington, C.J.; Garrard, T.L.; Stone, E.C. The abundance of the actinides in the cosmic radiation as measured on HEAO 3. Astrophys. J. Lett. 1982, 261, L117-L120. [CrossRef]

443. Binns, W.R.; Israel, M.H.; Klarmann, J.; Fickle, R.K.; Waddington, C.J.; Garrard, T.L.; Krombel, K.E.; Stone, E.C. Cosmic-ray abundances of Sn, Te, Xe, and BA nuclei measured on HEAO 3. Astrophys. J. Lett. 1983, 267, L93-L96. [CrossRef]

444. Binns, W.R.; Israel, M.H.; Brewster, N.R.; Fixsen, D.J.; Garrard, T.L. Lead, platinum, and other heavy elements in the primary cosmic radiation-HEAO 3 results. Astrophys. J. 1985, 297, 111-118. [CrossRef]

(C) 2020 by the authors. Licensee MDPI, Basel, Switzerland. This article is an open access article distributed under the terms and conditions of the Creative Commons Attribution (CC BY) license (http:/ / creativecommons.org/licenses/by/4.0/). 